\title{
WROŃSKIAN ALGEBRA AND BROADHURST-ROBERTS QUADRATIC RELATIONS
}

\author{
YAJUN ZHOU
}

To the memory of Dr. W (1986-2020)

\begin{abstract}
Through algebraic manipulations on Wrońskian matrices whose entries are reducible to Bessel moments, we present a new analytic proof of the quadratic relations conjectured by Broadhurst and Roberts, along with some generalizations. In the Wrońskian framework, we reinterpret the de Rham intersection pairing through polynomial coefficients in Vanhove's differential operators, and compute the Betti intersection pairing via linear sum rules for on-shell and off-shell Feynman diagrams at threshold momenta. From the ideal generated by Broadhurst-Roberts quadratic relations, we derive new non-linear sum rules for on-shell Feynman diagrams, including an infinite family of determinant identities that are compatible with Deligne's conjectures for critical values of motivic $L$-functions.
\end{abstract}

\section{CONTENTS}

1. Introduction

2. W algebra

2.1. Wrońskian matrices and Vanhove operators

2.2. Block diagonalization of on-shell Wrońskian matrices

2.3. Sum rules for on-shell and off-shell Bessel moments

3. $\mathbf{W}^{\star}$ algebra

3.1. Cofactors of Wrońskians

3.2. Threshold behavior of Wrońskian cofactors

3.3. Quadratic relations for off-shell Bessel moments $\quad 30$

4. Broadhurst-Roberts quadratic relations $\quad 37$

4.1. Quadratic relations for on-shell Bessel moments $\quad 37$

4.2. Arithmetic applications to Feynman diagrams

4.3. Alternative representations of de Rham matrices $\quad 49$

References

Date: May 18, 2021.

Keywords: Bessel moments, Feynman integrals, Wrońskian matrices, Bernoulli numbers

Subject Classification (AMS 2020): 11B68, 33C10, 34M35 (Primary) 81T18, 81T40 (Secondary)

* This research was supported in part by the Applied Mathematics Program within the Department of Energy (DOE) Office of Advanced Scientific Computing Research (ASCR) as part of the Collaboratory on Mathematics for Mesoscopic Modeling of Materials (CM4). 


\section{INTRODUCTION}

In perturbative quantum field theory, one frequently encounters Feynman diagrams in the form of on-shell Bessel moments [33, 29, 3, 32, 34, 9, 12, 10, 13, 14, 25]

$$
\operatorname{IKM}(a, b ; n):=\int_{0}^{\infty}\left[I_{0}(t)\right]^{a}\left[K_{0}(t)\right]^{b} t^{n} \mathrm{~d} t
$$

for certain non-negative integers $a, b, n \in \mathbb{Z}_{\geq 0}$, where $I_{0}(t)=\frac{1}{\pi} \int_{0}^{\pi} e^{t \cos \theta} \mathrm{d} \theta$ and $K_{0}(t)=\int_{0}^{\infty} e^{-t \cosh u} \mathrm{~d} u$ are modified Bessel functions of zeroth order. These Bessel moments satisfy a wealth of algebraic relations, which had been discovered by Broadhurst and collaborators through numerical experimentations [9, 12, 10, 13, 14], before becoming formally proven truths [50, 48, 49, 53, 54, 25].

The Broadhurst-Mellit determinant formulae [9, Conjectures 4 and 7] were among the earliest verified [49] non-linear algebraic relations for Bessel moments: the $k \times k$ matrices $\mathbf{M}_{k}:=(\mathbf{I K M}(a, 2 k+1-a ; 2 b-$ 1) $)_{1 \leq a, b \leq k}$ and $\mathbf{N}_{k}:=(\mathbf{I K M}(a, 2 k+2-a ; 2 b-1))_{1 \leq a, b \leq k}$ have determinants

$$
\operatorname{det} \mathbf{M}_{k}=\prod_{j=1}^{k} \frac{(2 j)^{k-j} \pi^{j}}{\sqrt{(2 j+1)^{2 j+1}}}, \quad \operatorname{det} \mathbf{N}_{k}=\frac{2 \pi^{(k+1)^{2} / 2}}{\Gamma((k+1) / 2)} \prod_{j=1}^{k+1} \frac{(2 j-1)^{k+1-j}}{(2 j)^{j}}
$$

where $\Gamma(x):=\int_{0}^{\infty} t^{x-1} e^{-t} \mathrm{~d} t$ for $x>0$.

These determinant formulae are refined by the Broadhurst-Roberts quadratic relations $\mathbf{B}_{m}=\mathbf{P}_{m} \mathbf{D}_{m} \mathbf{P}_{m}^{\mathrm{T}}$ [10, 13, 14] for the period matrix $\mathbf{P}_{m}:=\left((-1)^{b-1} \pi^{a-\frac{m+3}{2}} \mathbf{I K M}(a, m+2-a ; 2 b-1)\right)_{1 \leq a, b \leq\left\lfloor\frac{m+1}{2}\right\rfloor}$, in which the Betti matrix $\mathbf{B}_{m} \in \mathbb{Q}^{\left\lfloor\frac{m+1}{2}\right\rfloor \times\left\lfloor\frac{m+1}{2}\right\rfloor}$ and the de Rham matrix $\mathbf{D}_{m} \in \mathbb{Q}^{\left\lfloor\frac{m+1}{2}\right\rfloor \times\left\lfloor\frac{m+1}{2}\right\rfloor}$ are filled with rational numbers. Fresán, Sabbah and Yu have recently proved an equivalent form [25, Theorem 1.4] of the Broadhurst-Roberts quadratic relations, with an algebro-geometric tour de force that draws on their profound insights into certain exponential motives [22], whose associated Galois representations are intimately related to moments of Kloosterman sums [23, 24] (analogs of on-shell Bessel moments in positive characterisitic).

In this paper, we present an analytic proof of the Broadhurst-Roberts quadratic relations, by revisiting the Wrońskian method in our verification of the Broadhurst-Mellit determinant formulae [49]. Our Wrońskian-based proof also produces a new family of quadratic relations, which embody the Broadhurst-Roberts relations for on-shell Bessel moments as special cases.

In \$2, writing $D^{n} f(u):=\mathrm{d}^{n} f(u) / \mathrm{d} u^{n}$ for $n \in \mathbb{Z}_{>0}$, and $D^{0} f(u):=f(u)$, we will consider a Wrońskian matrix $\mathbf{W}\left[f_{1}(u), \ldots, f_{m}(u)\right]=\left(D^{i-1} f_{j}(u)\right)_{1 \leq i, j \leq m}$ whose entries are reducible to on-shell Bessel moments (more precisely, entries of $\mathbf{P}_{m}$ and $\mathbf{P}_{m-2}$ ) as $u \rightarrow 1^{-}$, and whose determinant $\operatorname{det} \mathbf{W}:=W\left[f_{1}(u), \ldots, f_{m}(u)\right]=$ $\operatorname{det}\left(D^{i-1} f_{j}(u)\right)_{1 \leq i, j \leq m}$ is explicitly computable through our proof of the Broadhurst-Mellit determinant formulae [49]. In \$3, our key observation is the adjugate relation $\mathbf{W}^{\star} \mathbf{W}=\mathbf{W} \mathbf{W}^{\star}=W \mathbf{I}_{m}$, where the matrix adjugate $\mathbf{W}^{\star}:=\operatorname{adj} \mathbf{W}$ of $\mathbf{W}\left[f_{1}(u), \ldots, f_{m}(u)\right]$ factorizes into $\mathbf{W}^{\star}=\mathbf{S} \mathbf{W}^{\mathrm{T}} \mathbf{U}$, with $\mathbf{S} \in \mathbb{Q}^{m \times m}$ and $\mathbf{U} / W \in \mathbb{Q}(u)^{m \times m}$. In $\$ 4$, we explore the $u \rightarrow 1^{-}$limit of the matrix equation $\mathbf{S} \mathbf{W}^{\mathrm{T}} \mathbf{U W}=W \mathbf{I}_{m}$, and establish quadratic relations among on-shell Bessel moments.

For a generic parameter $u \in(0,1)$, the individual elements of the Wrońskian matrix $\mathbf{W}\left[f_{1}(u), \ldots, f_{m}(u)\right]$ in $\$ 2$ will be representable by off-shell Bessel moments [49, Definition 2.1]

$$
\begin{aligned}
\widetilde{\mathbf{I K M}}(a+1, b ; n \mid u) & :=\int_{0}^{\infty} I_{0}(\sqrt{u} t)\left[I_{0}(t)\right]^{a}\left[K_{0}(t)\right]^{b} t^{n} \mathrm{~d} t, \\
\mathbf{\mathbf { K }} \mathbf{M}(a, b+1 ; n \mid u) & :=\int_{0}^{\infty} K_{0}(\sqrt{u} t)\left[I_{0}(t)\right]^{a}\left[K_{0}(t)\right]^{b} t^{n} \mathrm{~d} t,
\end{aligned}
$$


and differentiated Bessel moments [49, Definition 2.3]

$$
\begin{aligned}
& \operatorname{IKM}(a+1, b ; n \mid u):=+\int_{0}^{\infty} I_{1}(\sqrt{u} t)\left[I_{0}(t)\right]^{a}\left[K_{0}(t)\right]^{b} t^{n+1} \mathrm{~d} t, \\
& \mathbf{I K ́} \mathbf{K}(a, b+1 ; n \mid u):=-\int_{0}^{\infty} K_{1}(\sqrt{u} t)\left[I_{0}(t)\right]^{a}\left[K_{0}(t)\right]^{b} t^{n+1} \mathrm{~d} t,
\end{aligned}
$$

where $I_{1}(x)=+\mathrm{d} I_{0}(x) / \mathrm{d} x$ and $K_{1}(x)=-\mathrm{d} K_{0}(x) / \mathrm{d} x$. Accordingly, our efforts in $\S 3$ will culminate in a quadratic relation involving these generalized Bessel moments and some explicitly computable rational functions (in lieu of the rational numbers in the Betti matrix $\mathbf{B}$ and the de Rham matrix $\mathbf{D}$ of the Broadhurst-Roberts formulation), as stated below.

Theorem 1.1 (Wrońskian algebra). For $m \in \mathbb{Z}_{>0}, u \in\left(0, \frac{5-3(-1)^{m}}{2}\right)$, normalize off-shell Feynman diagrams as

$$
\mathcal{F}_{m, j}(u):= \begin{cases}\frac{\widetilde{\mathbf{I K M}}(1, m+1 ; 1 \mid u)+(m+1) \mathbf{\mathbf { K }} \mathbf{M}(1, m+1 ; 1 \mid u)}{(m+2) \pi^{(m+1) / 2}}, & j=1, \\ \frac{\widetilde{\mathbf{K} M}(j, m+2-j ; 1 \mid u)}{\pi^{(m+1) / 2+1-j}}, & j \in \mathbb{Z} \cap\left[2,\left\lfloor\frac{m}{2}\right\rfloor+1\right], \\ \frac{\mathbf{I} \mathbf{M}\left(j-\left\lfloor\frac{m}{2}\right\rfloor, m+2+\left\lfloor\frac{m}{2}\right\rfloor-j ; 1 \mid u\right)}{\pi^{(m+1) / 2+\lfloor m / 2\rfloor+1-j}}, & j \in \mathbb{Z} \cap\left[\left\lfloor\frac{m}{2}\right\rfloor+2, m\right],\end{cases}
$$

and define a polynomial

$$
\mathcal{L}_{m}(u):=u^{\left\lfloor\frac{m+1}{2}\right\rfloor} \prod_{\substack{n \in \mathbb{Z} \cap[1, m+1] \\ n \equiv m+1(\bmod 2)}}\left(u-n^{2}\right)
$$

where $\lfloor x\rfloor$ is the greatest integer less than or equal to $x$. For $u \in\left(0, \frac{5-3(-1)^{m}}{2}\right)$, the Wronskian matrix $\mathbf{W}_{m}(u):=\mathbf{W}\left[\mathcal{F}_{m, 1}(u), \ldots, \mathcal{F}_{m, m}(u)\right]$ has determinant

$$
W_{m}(u):=\operatorname{det} \mathbf{W}_{m}(u)=\frac{m+1}{m+2} \frac{(-1)^{\lfloor m / 4\rfloor}}{2^{m(m-1) / 2}} \frac{[(m+1) !]^{m}}{\prod_{n=1}^{m+1} n^{n}} \frac{1}{\left|\mathcal{L}_{m}(u)\right|^{m / 2}}
$$

and satisfies a quadratic relation

$$
\mathbf{W}_{m}(u) \mathbf{S}_{m} \mathbf{W}_{m}^{\mathrm{T}}(u)=\frac{\left[\mathbf{V}_{m}(u)\right]^{-1}}{\left|\mathcal{L}_{m}(u)\right|}
$$

for matrices $\mathbf{V}_{m}(u)=(-1)^{m+1} \mathbf{V}_{m}^{\mathrm{T}}(u) \in \mathbb{Q}(u)^{m \times m}$ and $\mathbf{S}_{m}=(-1)^{m+1} \mathbf{S}_{m}^{\mathrm{T}} \in \mathbb{Q}^{m \times m}$.

Explicitly, the Vanhove matrix $\mathbf{V}_{m}(u) \in \mathbb{Q}(u)^{m \times m}$ is upper-left triangular, with entries 1

$$
\left(\mathbf{V}_{m}(u)\right)_{a, b}:=\sum_{n=a+b-1}^{m}(-1)^{a+n+m+1}\left(\begin{array}{c}
n-a \\
b-1
\end{array}\right) \frac{D^{n-a-b+1} \ell_{m, n}(u)}{\mathcal{L}_{m}(u)}
$$

where the polynomials2 $\ell_{m, j}(u) \in \mathbb{Z}[u]$ are defined through the Vanhove-Verrill relation (cf. [43, \$3.6.1] and [44, §3.6.1]):

$$
\sum_{j=0}^{m} \ell_{m, j}(u) D^{j}:=u \widehat{\vartheta}^{m}+\sum_{k=1}^{\left\lfloor\frac{m}{2}\right\rfloor+1} u^{1-k} \sum_{\substack{n \in \mathbb{Z} \cap[1, k], \alpha_{k+1}=1 \\ \alpha_{n} \in \mathbb{Z} \cap[1, m+1] \\ \alpha_{n+1} \leq \alpha_{n}-2}}(\widehat{\vartheta}-k)^{m+1-\alpha_{1}} \prod_{n=1}^{k} \alpha_{n}\left(\alpha_{n}-m-2\right)(\widehat{\vartheta}-k+n)^{\alpha_{n}-\alpha_{n+1}}
$$

\footnotetext{
${ }^{1}$ Throughout this article, we write $\left(\begin{array}{l}n \\ m\end{array}\right):=\frac{n !}{m !(n-m) !}$ for the standard binomial coefficient with $n \in \mathbb{Z}_{\geq 0}, m \in \mathbb{Z} \cap[0, n]$. By convention, we also define $\left(\begin{array}{l}n \\ k\end{array}\right)=0$ when $n \in \mathbb{Z}_{\geq 0}, k \in \mathbb{Z} \backslash[0, n]$. Empty sums like $\sum_{n=A}^{B}(\cdots)$ with $A>B$ are treated as zero.

${ }^{2}$ These polynomials can be constructed by a finite enumeration over integer parameters $\alpha_{1}, \ldots, \alpha_{n}$ in (1.12). See Table 【 for a partial list of the Vanhove-Verrill polynomials.
} 
TABLE I. A partial list of Vanhove's operators $\widetilde{L}_{m}=\sum_{j=0}^{m} \ell_{m, j}(u) D^{j}$

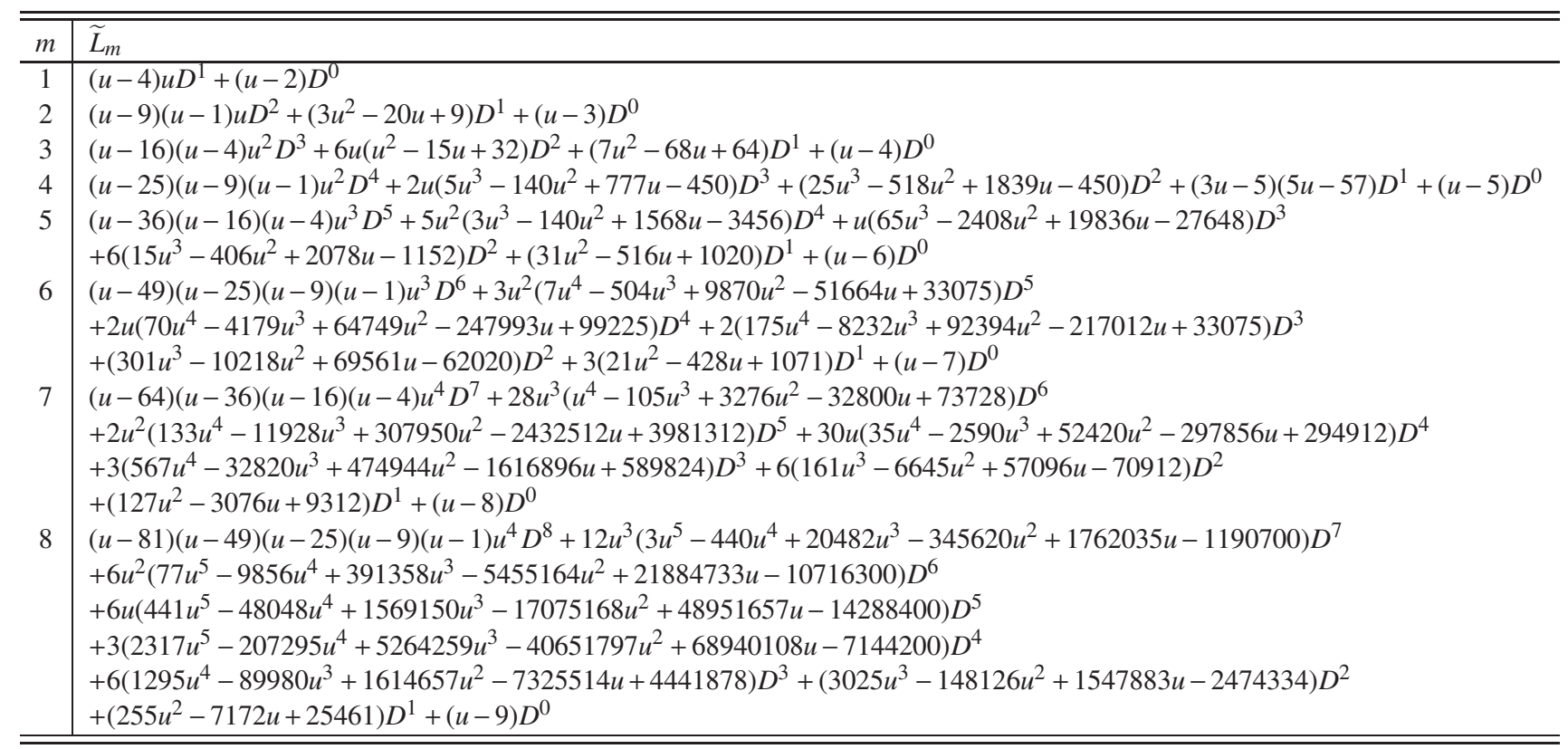

for $(\widehat{\vartheta} f)(u)=D^{1}[u f(u)]$; the matrix $\mathbf{S}_{m} \in \mathbb{Q}^{m \times m}$ can be partitioned into $\mathbf{S}_{m}=\left(\begin{array}{cc}\mathbf{S}_{m}^{A} & \mathbf{S}_{m}^{B} \\ \mathbf{S}_{m}^{C} & \mathbf{S}_{m}^{D}\end{array}\right)$ for

$$
\left(\mathbf{S}_{m}^{A}\right)_{a, b}:=\frac{1+(-1)^{a+b+m+1}}{2^{1-m}} \frac{\left[1+(m+1) \delta_{a, 1}\right]\left[1+(m+1) \delta_{b, 1}\right] \sum_{s=1}^{\left\lfloor\frac{m}{2}\right\rfloor+2-a}(-1)^{s}\left(\begin{array}{c}
m+2-a \\
\left\lfloor\frac{m+1}{2}\right\rfloor+s
\end{array}\right)\left(\begin{array}{c}
\left\lfloor\frac{m}{2}\right\rfloor+1-s \\
b-1
\end{array}\right)}{(-1)^{\left\lfloor\frac{a}{2}\right\rfloor+\left\lfloor\frac{b}{2}-\frac{1+(-1)^{m}}{4}\right\rfloor-\left\lfloor\frac{m}{2}\right\rfloor-1}(a-1) !(m+2-a) !}
$$

where $a, b \in \mathbb{Z} \cap\left[1,\left\lfloor\frac{m}{2}\right\rfloor+1\right]$,

$$
\begin{aligned}
& \left(\mathbf{S}_{m}^{B}\right)_{a, b^{\prime}}=(-1)^{m+1}\left(\mathbf{S}_{m}^{C}\right)_{b^{\prime}, a} \\
& :=\frac{1+(-1)^{a+b^{\prime}+m}}{2^{1-m}} \frac{\left[1+(m+1) \delta_{a, 1}\right] \sum_{s=1}^{\left\lfloor\frac{m}{2}\right\rfloor+2-a}(-1)^{s}\left(\begin{array}{c}
m+2-a \\
\left\lfloor\frac{m+1}{2}\right\rfloor+s
\end{array}\right)\left[\left(\begin{array}{c}
\left\lfloor\frac{m}{2}\right\rfloor+1-s \\
b^{\prime}+1
\end{array}\right)+(-1)^{b^{\prime}}\left(\begin{array}{l}
\left\lfloor\frac{m+1}{2}\right\rfloor+s \\
b^{\prime}+1
\end{array}\right)\right]}{(-1)^{(a-1) m+\left\lfloor\frac{a}{2}-\frac{1+(-1)^{m}}{4}\right\rfloor+\left\lfloor\frac{b^{\prime}}{2}+\frac{1+(-1)^{m}}{4}\right\rfloor-\left\lfloor\frac{m+1}{2}\right\rfloor}(a-1) !(m+2-a) !}
\end{aligned}
$$

where $a \in \mathbb{Z} \cap\left[1,\left\lfloor\frac{m}{2}\right\rfloor+1\right], b^{\prime} \in \mathbb{Z} \cap\left[1,\left\lfloor\frac{m-1}{2}\right\rfloor\right]$, and

$$
\left(\mathbf{S}_{m}^{D}\right)_{a^{\prime}, b^{\prime}}:=\frac{1+(-1)^{m+1}}{2} \frac{(-4)^{\left\lfloor\frac{m+1}{2}\right\rfloor-1}}{\left(\left\lfloor\frac{m+1}{2}\right\rfloor !\right)^{2}} \frac{\left[1+(-1)^{a^{\prime}}\right]\left[1+(-1)^{b^{\prime}}\right]}{(-1)^{\left\lfloor\frac{a^{\prime}}{2}\right\rfloor+\left\lfloor\frac{b^{\prime}}{2}\right\rfloor}}\left(\begin{array}{c}
\left\lfloor\frac{m+1}{2}\right\rfloor \\
a^{\prime}+1
\end{array}\right)\left(\begin{array}{c}
\left\lfloor\frac{m+1}{2}\right\rfloor \\
b^{\prime}+1
\end{array}\right)
$$

where $a^{\prime}, b^{\prime} \in \mathbb{Z} \cap\left[1,\left\lfloor\frac{m-1}{2}\right\rfloor\right]$.

In $\$ 4$, we push the theorem above to the $u \rightarrow 1^{-}$limit, in the following form.

Theorem 1.2 (Broadhurst-Roberts quadratic relations). Define the Bessel-Feynman moments $\mathcal{F}_{m, j}^{\ell}(u)$ by

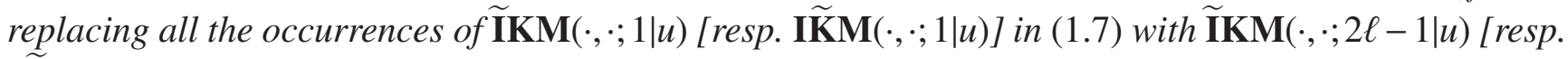
IKMM( $(\cdot, ; 2 \ell-1 \mid u)]$. For each $m \in \mathbb{Z}_{>1}$, the Broadhurst-Roberts period matrix $\mathbf{P}_{m}:=$ $\left((-1)^{b-1} \mathcal{F}_{m, a}^{b}(1)\right)_{1 \leq a, b \leq\left\lfloor\frac{m+1}{2}\right\rfloor}$ satisfies a quadratic relation

$$
\mathbf{P}_{m} \mathbf{D}_{m} \mathbf{P}_{m}^{\mathrm{T}}=\mathbf{B}_{m},
$$

for de Rham matrix $\mathbf{D}_{m}=(-1)^{m+1} \mathbf{D}_{m}^{\mathrm{T}} \in \mathbb{Q}^{\left\lfloor\frac{m+1}{2}\right\rfloor \times\left\lfloor\frac{m+1}{2}\right\rfloor}$ and Betti matrix $\mathbf{B}_{m}=(-1)^{m+1} \mathbf{B}_{m}^{\mathrm{T}} \in \mathbb{Q}^{\left\lfloor\frac{m+1}{2}\right\rfloor \times\left\lfloor\frac{m+1}{2}\right\rfloor}$.

\footnotetext{
${ }^{3}$ The Kronecker delta is defined as $\delta_{m, n}=1$ when $m=n$ and $\delta_{m, n}=0$ when $m \neq n$.
} 
Explicitly, the de Rham matrix $\mathbf{D}_{m-2}$ is upper-left triangular, with entries

$$
\left(\mathbf{D}_{m-2}\right)_{a, b}:=\lim _{u \rightarrow 1^{-}} \frac{\left|\mathcal{L}_{m}(u)\right|\left(\left(\boldsymbol{\beta}_{m}^{-1}\right)^{\mathrm{T}} \mathbf{V}_{m}(u) \boldsymbol{\beta}_{m}^{-1}\right)_{a+\left\lfloor\frac{m-1}{2}\right\rfloor+1, b+\left\lfloor\frac{m-1}{2}\right\rfloor+1}}{4(m+2)(-1)^{\left\lfloor\frac{m-1}{2}\right\rfloor}}
$$

computable via the Vanhove matrix $\mathbf{V}_{m}(u)$ [see (1.11)] and the Bessel matrix $\boldsymbol{\beta}_{m} \in \mathbb{Z}^{m \times m}$ for

$$
\left(\boldsymbol{\beta}_{m}\right)_{a, b}:= \begin{cases}(-4)^{a-1} \frac{(a-1) !}{(b-a) !}\left(\begin{array}{c}
a-1 \\
b-a
\end{array}\right), & a \in \mathbb{Z} \cap\left[1,\left\lfloor\frac{m+1}{2}\right\rfloor\right], \\
(-4)^{a-\left\lfloor\frac{m+1}{2}\right\rfloor-1} \frac{2\left(a-\left\lfloor\frac{m+1}{2}\right\rfloor-1\right) !}{\left(b-a+\left\lfloor\frac{m+1}{2}\right\rfloor-1\right) !}\left(\begin{array}{c}
a-\left\lfloor\frac{m+1}{2}\right\rfloor \\
b-a+\left\lfloor\frac{m+1}{2}\right\rfloor
\end{array}\right), & a \in \mathbb{Z} \cap\left[\left\lfloor\frac{m+1}{2}\right\rfloor+1, m\right] ;\end{cases}
$$

the Betti matrix $\mathbf{B}_{m-2}$ satisfies

$$
\begin{aligned}
\left(\mathbf{B}_{m-2}\right)_{a, b} & :=\frac{\left(\mathbf{S}_{m}^{-1}\right)_{a+\left\lfloor\frac{m}{2}\right\rfloor+1, b+\left\lfloor\frac{m}{2}\right\rfloor+1}-\left(\mathbf{S}_{m}^{-1}\right)_{a+1, b+\left\lfloor\frac{m}{2}\right\rfloor+1}-\left(\mathbf{S}_{m}^{-1}\right)_{a+\left\lfloor\frac{m}{2}\right\rfloor+1, b+1}}{4(m+2)(-1)^{\left\lfloor\frac{m-1}{2}\right\rfloor}} \\
& =\frac{(-1)^{a+1}}{2^{m+1}} \frac{(m-a) !(m-b) !}{(m+1-a-b) !} \frac{\mathrm{B}_{m+1-a-b}}{(-1)^{\left\lfloor\frac{m-a-b}{2}\right\rfloor}}
\end{aligned}
$$

where $\mathbf{S}_{m}=\left(\begin{array}{ll}\mathbf{S}_{m}^{A} & \mathbf{S}_{m}^{B} \\ \mathbf{S}_{m}^{C} & \mathbf{S}_{m}^{D}\end{array}\right)=(-1)^{m+1} \mathbf{S}_{m}^{\mathrm{T}}$ is defined through (1.13)-(1.15), and the Bernoulli numbers $\mathrm{B}_{n}, n \in$ $\mathbb{Z}_{\geq 0}$ are generated by

$$
\frac{t}{e^{t}-1}:=\sum_{n=0}^{\infty} \mathrm{B}_{n} \frac{t^{n}}{n !}, \quad|t|<\pi .
$$

We note that Lee and Pomeransky [35, Appendix C] have recently produced some quadratic relations for off-shell Bessel moments, but not for differentiated Bessel moments. There are technical difficulties when one attempts to push their results to the on-shell limit [35, §6.4] and recover Broadhurst-Roberts quadratic relations therefrom.

For each $m \in \mathbb{Z}_{>1}$, our Betti matrix $\mathbf{B}_{m}$ agrees with the original formulation of Broadhurst-Roberts [13, (5.4)-(5.6)], up to reordering of rows and columns. Instead of considering Betti intersection pairing that inspired the conjecture of Broadhurst-Roberts [10, 13, 14] and the proof by Fresán-Sabbah-Yu [25, Theorem 1.4], we generate $\mathbf{B}_{m}$ by inverting $\mathbf{S}_{m+2}$. The latter matrix $\mathbf{S}_{m+2}$ draws on explicit sum rules (with rational coefficients) for on-shell and off-shell Bessel moments, which extend our earlier works [50, 53, 52] on the same topic.

In addition to elucidating the relations between Betti matrices and sum rules for individual Bessel moments in $\$ 4.1$ we will also establish a family of sum rules for minor determinants of $\mathbf{M}_{k}, k \in \mathbb{Z}_{>1}$ in $\$ 4.2$ (see Proposition 4.5 for details):

$$
\begin{aligned}
& \operatorname{det}(\mathbf{I K M}(2 a, 2(k-a)+1 ; 2 b-1))_{1 \leq a, b \leq\left\lfloor\frac{k}{2}\right\rfloor} \\
= & \frac{1}{\pi^{\left\lfloor\frac{k+1}{2}\right\rfloor}} \frac{\sqrt{[(2 k+1) ! !]^{2-(-1)^{k}}}}{2^{\left\lfloor\frac{k}{2}\right\rfloor}(k-1) ! !(k ! !)^{1-(-1)^{k}}} \operatorname{det}(\mathbf{I K M}(2 a-1,2(k-a+1) ; 2 b-1))_{1 \leq a, b \leq\left\lfloor\frac{k+1}{2}\right\rfloor} .
\end{aligned}
$$

Such sum rules may have some arithmetic interest: Deligne's conjecture [19] for the motive $\mathrm{M}_{2 k+1}$ (as defined in $[25, \S 7 . c])$ predicts that the determinants on both sides of $(1.21)$ are $\mathbb{Q}^{\times} \pi^{\mathbb{Z}}$ multiples of critical $L$-values, while these critical values for $L\left(\mathrm{M}_{2 k+1}, s\right)$ are related to each other by a functional equation [23, Theorem 1.2]. No such analogs appear to exist in minor determinants of $\mathbf{N}_{k}, k \in \mathbb{Z}_{>1}$, for both algebraic and analytic reasons.

Our algorithmic construction of the de Rham matrix $\mathbf{D}_{m}$ is based on derivatives of the polynomials $\ell_{m+2, j}(u)$ at $u=1$, which are readily adaptable to systems different from Bessel moments (see \$3.1). Within the same Wrońskian framework, one can establish several other equivalent representations [see 
(4.76) - 4.79) for details] of $\mathbf{D}_{m}$, along with new algebraic relations [see (4.80) for details] connecting the period matrix $\mathbf{P}_{m}$ to

$$
\mathbf{P}_{o}:=\left(\frac{(-1)^{b-1}}{\pi^{\frac{m+3}{2}-a}} \int_{0}^{\infty}\left\{\frac{\left[I_{0}(t)\right]^{a} \log t}{\left[K_{0}(t)\right]^{a}}-\frac{\delta_{a, 1}}{m+2}\right\}\left[K_{0}(t)\right]^{m+2} t^{2 b-1} \mathrm{~d} t\right)_{1 \leq a, b \leq\left\lfloor\frac{m+1}{2}\right\rfloor} .
$$

Outside our Wrońskian framework, there are still other approaches to de Rham representations, such as the Broadhurst-Roberts combinatorial recursion formulae [13, (5.7)-(5.12)] and the Fresán-Sabbah-Yu self-duality pairing [25, §3.b]. A direct proof of the equivalence between Wrońskian and non-Wrońskian approaches to de Rham matrices will not appear in this article. Nevertheless, we will outline general principles that may reconcile our representation of de Rham matrices with those of Broadhurst-Roberts and Fresán-Sabbah-Yu, in $\$ 4.3$.

Acknowledgments The author thanks David Broadhurst and Javier Fresán for their feedback on the first draft. The author is grateful to Pierre Vanhove for his invitation to Séminaire Motifs et intégrales de Feynman. The author is indebted to two anonymous referees for their suggestions on improving the presentation of this manuscript.

\section{W ALGEBRA}

In $\$ 2.1$, we open by recapitulating our previous work [49] on the Broadhurst-Mellit determinant formulae, with an extended discussion on the ordinary differential equations satisfied by off-shell Bessel moments $\mathcal{F}_{m, j}(u)$.

In $\$ 2.2$, we elucidate the relation between Wrońskian matrices and on-shell Bessel moments. We will achieve this by studying the Wrońskian matrices $\mathbf{W}_{m}(u):=\mathbf{W}\left[\mathcal{F}_{m, 1}(u), \ldots, \mathcal{F}_{m, m}(u)\right]$ for $m \in \mathbb{Z}_{>0}$ in the $u \rightarrow 1^{-}$limit, similar to procedures reported in [49].

In $\$ 2.3$, we establish, among other things, several families of $\mathbb{Q}$-linear dependence relations concerning on-shell and off-shell Bessel moments, when the parameter $u$ is a perfect square. This sets the stage for the analysis of the adjugate matrices $\mathbf{W}_{m}^{\star}(u)$ for $m \in \mathbb{Z}_{>0}$, which will unfold in $\$ 3$.

2.1. Wrońskian matrices and Vanhove operators. For $m \in \mathbb{Z}_{>0}$, consider the functions $\mathcal{F}_{m, j}(u), j \in$ $\mathbb{Z} \cap[1, m]$ introduced in (1.7), which are normalized versions of the notations in [49, Definition 4.1]. In our previous work [49, §4], we have manipulated Wrońskian matrices $\mathbf{W}_{m}(u):=\mathbf{W}\left[\mathcal{F}_{m, 1}(u), \ldots, \mathcal{F}_{m, m}(u)\right]$ of all sizes, and have shown that their determinants $W_{m}(u):=\operatorname{det} \mathbf{W}_{m}(u)$ [see (1.9)] satisfy (cf. [49, (4.36) and (4.62)]):

$$
\begin{gathered}
W_{2 k-1}(u)=\frac{(-1)^{\frac{(k-1)(k-2)}{2}} k[\Gamma(k / 2)]^{2}}{\pi^{k^{2}} u^{k(2 k-1) / 2}(2 k+1)} \frac{\left(\operatorname{det} \mathbf{N}_{k-1}\right)^{2}}{2^{(k-1)(2 k-1)+1}} \prod_{j=1}^{k}\left[\frac{(2 j)^{2}}{(2 j)^{2}-u}\right]^{k-\frac{1}{2}}, \quad \forall u \in(0,4), \\
W_{2 k}(u)=\frac{(-1)^{\frac{k(k-1)}{2}}}{\pi^{k(k+1)}} \frac{(2 k+1)\left(\operatorname{det} \mathbf{M}_{k}\right)^{2}}{2^{(2 k-1) k+1} u^{k^{2}}(k+1)} \prod_{j=1}^{k+1}\left[\frac{(2 j-1)^{2}}{(2 j-1)^{2}-u}\right]^{k}, \quad \forall u \in(0,1),
\end{gathered}
$$

for each $k \in \mathbb{Z}_{\geq 2}$. In particular, these collateral results for the Broadhurst-Mellit determinant formulae (1.2) tell us that the Wrońskian matrix $\mathbf{W}_{m}(u)$ is non-singular for $u \in\left(0, \frac{5-3(-1)^{m}}{2}\right)$, hence the linear independence of the functions $\mathcal{F}_{m, 1}(u), \ldots, \mathcal{F}_{m, m}(u)$ on the same interval.

Furthermore, we may deduce from [49, Lemma 4.2] that the aforementioned linearly independent functions form complete sets of fundamental solutions to certain ordinary differential equations $4 C^{\infty}(0$, $\left.\frac{5-3(-1)^{m}}{2}\right) \cap \operatorname{ker} \widetilde{L}_{m}=\operatorname{span}_{\mathbb{C}}\left\{\mathcal{F}_{m, j}(u), u \in\left(0, \frac{5-3(-1)^{m}}{2}\right) \mid j \in \mathbb{Z} \cap[1, m]\right\}$, where Vanhove's operator $\widetilde{L}_{m}$ assumes the following form (omitting lower order terms) [42, (9.11)-(9.12)]:

$$
\widetilde{L}_{m}=\mathcal{L}_{m}(u) D^{m}+\frac{m}{2} \frac{\mathrm{d} \mathcal{L}_{m}(u)}{\mathrm{d} u} D^{m-1}+\cdots,
$$

\footnotetext{
${ }^{4}$ Using the same techniques of integration by parts as in [49, Lemma 4.2], one can show that $\widetilde{L}_{m}$ annihilates I $\widetilde{\mathbf{K}} \mathbf{M}(a, m+2-$ $a ; 1 \mid u)$ for $a \in \mathbb{Z} \cap[2, m+1]$, when $u$ belongs to a certain open interval that ensures the convergence of the respective integral.
} 
with the polynomial $\mathcal{L}_{m}(u)$ defined in (1.8). The leading and sub-leading terms in (2.3) explain why $W_{m}(u)$ is a constant multiple of $\left|\mathcal{L}_{m}(u)\right|^{-m / 2}$ [see [1.9] ], according to standard manipulations of Wrońskian determinants [31, §5.2, p. 119].

In general, Vanhove's operator $\widetilde{L}_{m}, m \in \mathbb{Z}_{>0}$ takes the form $\widetilde{L}_{m}=\sum_{j=0}^{m} \ell_{m, j}(u) D^{j}$, where $\ell_{m, j}(u) \in \mathbb{Z}[u]$. These polynomials $\ell_{m, j}(u)$ can be deduced from the closed-form representations in the next proposition, which in turn is amplified and extrapolated from [43, §3.6.1] and [44, §3.6.1].

Proposition 2.1 (Vanhove's operators in explicit form). For each $m \in \mathbb{Z}_{>0}$, we normalize Verrill's polynomials as $\mathscr{V}_{m, 0}(t)=t^{m}$ and

$$
\begin{aligned}
& \mathscr{V}_{m, k}(t):=t^{m} \sum_{\substack{n \in \mathbb{Z} \cap[1, k] \\
\alpha_{n} \in \mathbb{Z} \cap[1, m+1] \\
\alpha_{n+1} \leq \alpha_{n}-2}} \prod_{n=1}^{k} \alpha_{n}\left(\alpha_{n}-m-2\right)\left(\frac{t-n}{t-n+1}\right)^{\alpha_{n}-1} \\
& =\sum_{\substack{n \in \mathbb{Z} \cap[1, k], \alpha_{k+1}=1 \\
\alpha_{n} \in \mathbb{Z} \cap[1, m+1] \\
\alpha_{n+1} \leq \alpha_{n}-2}} t^{m+1-\alpha_{1}} \prod_{n=1}^{k} \alpha_{n}\left(\alpha_{n}-m-2\right)(t-n)^{\alpha_{n}-\alpha_{n+1}} \in \mathbb{Z}[t]
\end{aligned}
$$

for $k \in \mathbb{Z}_{>0}$, with empty sums being 0 and empty products being 1 .

If we define $(\widehat{\vartheta} f)(u)=D^{1}[u f(u)]$ and write $\lfloor x\rfloor$ for the greatest integer less than or equal to $x$, then we have the following explicit representation for Vanhove's differential operator of $m$-th order:

$$
\widetilde{L}_{m}=(-1)^{m} \sum_{k=0}^{\left\lfloor\frac{m}{2}\right\rfloor+1} u^{1-k} \mathscr{V}_{m, k}(k-\widehat{\vartheta})
$$

Proof. Using the arguments in [49, Lemma 4.2], one can show that Vanhove's operator $\widetilde{L}_{m}$ annihilates

$$
\mathbf{I \mathbf { K }} \mathbf{M}(m+1,1 ; 1 \mid u):=\int_{0}^{\infty} K_{0}(\sqrt{u} t)\left[I_{0}(t)\right]^{m+1} t \mathrm{~d} t, \quad u \in\left((m+1)^{2}, \infty\right) .
$$

Thanks to a convergent Taylor series [7, (2.4)]

$$
\left[I_{0}(t)\right]^{m+1}=\sum_{k=0}^{\infty} \mathrm{W}_{m+1}(2 k)\left(\frac{t^{k}}{2^{k} k !}\right)^{2}, \text { where } \mathrm{W}_{m+1}(2 k)=\sum_{\substack{a_{1}, a_{2}, \ldots, a_{m+1} \in \mathbb{Z}_{\geq 0} \\ a_{1}+a_{2}+\cdots+a_{m+1}=k}}\left(\frac{k !}{a_{1} ! a_{2} ! \cdots a_{m+1} !}\right)^{2}
$$

we may invoke the dominated convergence theorem, and integrate termwise, as done by Glasser-Montaldi [28, (A3)]:

$$
\mathbf{I \mathbf { K }} \mathbf{M}(m+1,1 ; 1 \mid u)=\int_{0}^{\infty} K_{0}(\sqrt{u} t)\left[\sum_{k=0}^{\infty} \mathrm{W}_{m+1}(2 k)\left(\frac{t^{k}}{2^{k} k !}\right)^{2}\right] t \mathrm{~d} t=\sum_{k=0}^{\infty} \frac{\mathrm{W}_{m+1}(2 k)}{u^{k+1}} .
$$

We write the right-hand side of (2.5) as $\widetilde{R}_{m}=\sum_{j=0}^{m} r_{m, j}(u) D^{j}$, with $r_{m, j}(u) \in \mathbb{Q}(u)$ being rational functions in $u$. By virtue of Verrill's recursion [45, Theorem 1] $\sum_{k=0}^{\left\lfloor\frac{m}{2}\right\rfloor+1} \mathscr{V}_{m, k}(n) \mathrm{W}_{m+1}(2(n-k))=0$, we know that $\widetilde{R}_{m}$ annihilates $\underset{\widetilde{\mathbf{K}} \mathbf{M}}{\widetilde{R}}(m+1,1 ; 1 \mid u), u \in\left((m+1)^{2}, \infty\right)$, so there exists a rational function $\rho(u) \in \mathbb{Q}(u)$ such that $\rho(u) \widetilde{L}_{m}=\widetilde{R}_{m}$. Moreover, we recover Vanhove's leading term $\mathcal{L}_{m}(u) D^{m}$ in (2.3) from

$$
r_{m, m}(u)=u^{m+1}+u^{\left\lfloor\frac{m+1}{2}\right\rfloor} \sum_{k=1}^{\left\lfloor\frac{m}{2}\right\rfloor+1} u^{\left\lfloor\frac{m}{2}\right\rfloor+1-k} \sum_{\substack{n \in \mathbb{Z} \cap[1, k] \\ \alpha_{n} \in \mathbb{Z} \cap[1, m+1] \\ \alpha_{n+1} \leq \alpha_{n}-2}} \prod_{n=1}^{k} \alpha_{n}\left(\alpha_{n}-m-2\right)=u^{\left\lfloor\frac{m+1}{2}\right\rfloor} \prod_{\substack{n \in \mathbb{Z} \cap[1, m+1] \\ n \equiv m+1(\bmod 2)}}\left(u-n^{2}\right)
$$

where the last combinatorial identity owes to Djakov-Mityagin (see [20, Theorem 4.1] or [21, Theorem 8]) and Zagier [7, Theorem 2.7]. Hence $\rho(u)=1$ and $\mathcal{L}_{m}(u)=\ell_{m, m}(u)$. 
Remark For an equivalent formulation of Vanhove's operator in the variable $s=\frac{1}{u}$ and its proof, see the recent work of Bönisch-Fischbach-Klemm-Nega-Safari [17, §3.1].

By Vanhove's original construction of $\widetilde{L}_{m}=\sum_{j=0}^{m} \ell_{m, j}(u) D^{j}$ in [42, §9], we know that $\ell_{m, j}(u)$ is always a polynomial in $u$ with integer coefficients. In the next corollary, we will use the explicit formula (2.5) to show that such polynomials have certain divisibility properties, thereby paving the way for the asymptotic analysis (to be elaborated in Proposition 2.10) of solutions to Vanhove's differential equations in the $u \rightarrow 0^{+}$regime.

Corollary 2.2 (Regular singular point). For all $m \in \mathbb{Z}_{>0}$, we have $u^{m-j-\left\lfloor\frac{m+1}{2}\right\rfloor} \ell_{m, j}(u) \in \mathbb{Z}[u]$ for $j \in \mathbb{Z} \cap$ $[0, m]$. As a consequence, for each $m \in \mathbb{Z}_{>0}$, the point $u=0$ is a regular singular point for Vanhove's differential equation $\widetilde{L}_{m} f(u)=0$, so that for a suitably chosen $\delta_{m} \in(0,1)$, every solution $f(u), u \in\left(0, \delta_{m}\right)$ is expressible as a convergent series in (fractional) powers of $u$ and $\log u$.

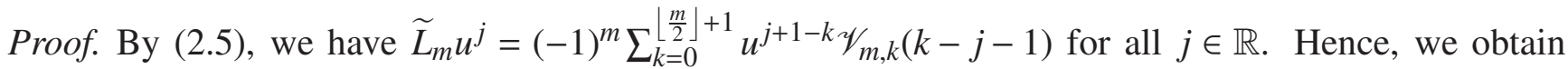
$\lim _{u \rightarrow 0^{+}} u^{m+1-j-\left\lfloor\frac{m+1}{2}\right\rfloor} \widetilde{L}_{m} u^{j}=0$.

From $\widetilde{L}_{m}=\sum_{j=0}^{m} \ell_{m, j}(u) D^{j}$, we have $u^{m+1-j-\left\lfloor\frac{m+1}{2}\right\rfloor} \widetilde{L}_{m} u^{j}=u^{m+1-j-\left\lfloor\frac{m+1}{2}\right\rfloor} \sum_{n=0}^{j} \ell_{m, n}(u) D^{n} u^{j}$ for $j \in \mathbb{Z} \cap$ $[0, m]$. In view of the output from the last paragraph, we can deduce $\lim _{u \rightarrow 0^{+}} u^{m+1-j-\left\lfloor\frac{m+1}{2}\right\rfloor} \ell_{m, j}(u)=0$ for all $j \in \mathbb{Z} \cap[0, m]$, beginning with the $j=0$ case, and proceeding with finitely many recursions. This shows that $u^{m-j-\left\lfloor\frac{m+1}{2}\right\rfloor} \ell_{m, j}(u) \in \mathbb{Z}[u]$ for $j \in \mathbb{Z} \cap[0, m]$, as claimed.

Now that $u^{m-j} \ell_{m, j}(u) / \ell_{m, m}(u)$ is holomorphic in a non-void $\mathbb{C}$-neighborhood of $u=0$ for every $j \in$ $\mathbb{Z} \cap[0, m-1]$, we know that the differential equation $\widetilde{L}_{m} f(u)=0, u \in(0,1)$ has a regular singular point at $u=0$, according to the Fuchs condition [31, §15.3]. The existence of generalized power series expansion then follows immediately.

For any differential operator $\widehat{O}=\sum_{k=0}^{n} a_{k}(u) D^{k}$ with $a_{k} \in C^{\infty}(a, b), k \in \mathbb{Z} \cap[0, n]$, its formal adjoint (or "formal dual") $\widehat{O}^{*}$ acts on every smooth function $f \in C^{\infty}(a, b)$ in the following manner: $\widehat{O}^{*} f(u)=$ $\sum_{k=0}^{n}(-1)^{k} D^{k}\left[a_{k}(u) f(u)\right]$. This formal adjoint is the unique operator satisfying $\langle\widehat{O} f, g\rangle_{(a, b)}=\left\langle f, \widehat{O}^{*} g\right\rangle_{(a, b)}$ for every smooth function $f \in C^{\infty}(a, b)$ and every compactly supported smooth function $g \in C_{c}^{\infty}(a, b)$, where $\langle f, g\rangle_{(a, b)}=\int_{a}^{b} f(u) g(u) \mathrm{d} u$.

We study the formal adjoint of each Vanhove operator in the next proposition, which will become useful later in Proposition 3.1 .

Proposition 2.3 (Parity of Vanhove operators). For every $m \in \mathbb{Z}_{>1}$, we have $\widetilde{L}_{m}^{*}=(-1)^{m} \widetilde{L}_{m}$.

Proof. We compute each summand in

$$
\widetilde{L}_{m}^{*}=(-1)^{m} \sum_{k=0}^{\left\lfloor\frac{m}{2}\right\rfloor+1}\left[u^{1-k} \mathscr{V}_{m, k}(k-\widehat{\vartheta})\right]^{*}
$$

separately.

For $k=0$, we have $\left[u \mathscr{V}_{m, 0}(-\widehat{\vartheta})\right]^{*}=(-1)^{m}\left(u \widehat{\vartheta}^{m}\right)^{*}=u \widehat{\vartheta}^{m}$ through integration by parts.

For $k \in \mathbb{Z}_{>0}$, we operate on power functions $u^{-1-r}, r \in \mathbb{R}$ :

$$
\begin{aligned}
u^{1-k} \mathscr{V}_{m, k}(k-\widehat{\vartheta}) \frac{1}{u^{r+1}} & =\sum_{\substack{n \in \mathbb{Z} \cap[1, k], \alpha_{0}=m+1, \alpha_{k+1}=1 \\
\alpha_{n} \in \mathbb{Z} \cap[1, m+1], \alpha_{n+1} \leq \alpha_{n}-2}} \frac{\prod_{n=1}^{k} \alpha_{n}\left(\alpha_{n}-m-2\right)}{u^{r+k}} \prod_{j=0}^{k}(r+k-j)^{\alpha_{j}-\alpha_{j+1}}, \\
(-1)^{m}\left[u^{1-k} \mathscr{V}_{m, k}(k-\widehat{\vartheta})\right]^{*} \frac{1}{u^{r+1}} & =\sum_{\substack{n \in \mathbb{Z} \cap[1, k], \beta_{0}=m+1, \beta_{k+1}=1 \\
\beta_{n} \in \mathbb{Z} \cap\left[1, m+1, \beta_{n+1}-\beta_{n}-2\right.}} \frac{\prod_{n=1}^{k} \beta_{n}\left(\beta_{n}-m-2\right)}{u^{r+k}} \prod_{j=0}^{k}(r+j)^{\beta_{j}-\beta_{j+1}} .
\end{aligned}
$$


One can identify the left-hand sides of the last two equations, by pairing up the dummy indices $\beta_{n}=$ $m+2-\alpha_{k+1-n}$ for $n \in \mathbb{Z} \cap[1, k]$.

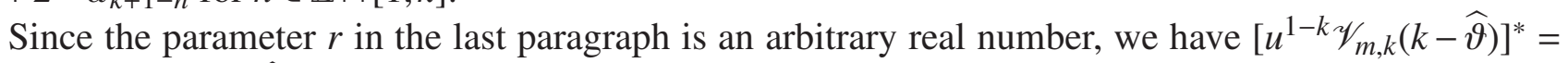
$(-1)^{m} u^{1-k} \mathscr{V}_{m, k}(k-\widehat{\vartheta})$ for $k \in \mathbb{Z}_{\geq 0}$, finally leading us to the claimed parity relation $\widetilde{L}_{m}^{*}=(-1)^{m} \widetilde{L}_{m}$.

An immediate consequence of the last proposition is a combinatorial constraint on the coefficients $\ell_{m, j}(u)$ in Vanhove's operator $\widetilde{L}_{m}=\sum_{j=0}^{m} \ell_{m, j}(u) D^{j}$ for each $m \in \mathbb{Z}_{>1}$.

Corollary 2.4 (Combinatorial constraint on $\ell_{m, j}(u)$ ). For each $m \in \mathbb{Z}_{>1}$, we have

$$
(-1)^{m} \ell_{m, j}(u)=\sum_{n=j}^{m}(-1)^{n}\left(\begin{array}{l}
n \\
j
\end{array}\right) D^{n-j} \ell_{m, n}(u)
$$

for $j \in \mathbb{Z} \cap[0, m]$, which incorporates Vanhove's sub-leading term [cf. (2.3)]

$$
\ell_{m, m-1}(u)=\frac{m}{2} D^{1} \ell_{m, m}(u)
$$

as a special case.

Proof. With the product rule for differentiation, we compute

$$
(-1)^{m} \widetilde{L}_{m} f(u)=\widetilde{L}_{m}^{*} f(u)=\sum_{n=0}^{m}(-1)^{n} D^{n}\left[\ell_{m, n}(u) f(u)\right]=\sum_{n=0}^{m}(-1)^{n} \sum_{j=0}^{n}\left(\begin{array}{l}
n \\
j
\end{array}\right)\left[D^{n-j} \ell_{m, n}(u)\right] D^{j} f(u),
$$

whose coefficients for $D^{j} f(u)$ boil down to (2.12).

For $j=m-1$, the constraint (2.12) reduces to $(-1)^{m} \ell_{m, m-1}(u)=(-1)^{m} m D^{1} \ell_{m, m}(u)+(-1)^{m-1} \ell_{m, m-1}(u)$, which is equivalent to (2.13), a vital result for the computations of Wrońskian determinants.

Remark We refer our readers to Corollary 3.3 in $\$ 3.1$, for non-trivial applications of the generic recursion (2.12).

During our proof of Proposition 2.1, we have considered solutions to Vanhove's differential equations $\widetilde{L}_{m} f(u)=0, u \in(m+1, \infty)$. Later on in $\S \$ 3.2 \sqrt{3.3}$, we will also find it helpful to solve $\widetilde{L}_{m} f(u)=0, u \in$ $\mathbb{R} \backslash\left\{\left(2 j-\frac{1+(-1)^{m}}{2}\right)^{2} \mid j \in \mathbb{Z} \cap\left[1,\left\lfloor\frac{m}{2}\right\rfloor+1\right]\right\}$ for each $m \in \mathbb{Z}_{>0}$. To solve Vanhove's differential equations in such scenarios, we need to extend our previous notations for the Bessel-Feynman moments $\mathcal{F}_{m, j}^{\ell}(u)$ to $\mathcal{F}_{m, j}^{\ell}(u)=\pi^{j-(m+1) / 2-\left\lfloor\frac{m}{2}\right\rfloor-1} \mathbf{I} \widetilde{\mathbf{K}} \mathbf{M}\left(j-\left\lfloor\frac{m}{2}\right\rfloor, m+2+\left\lfloor\frac{m}{2}\right\rfloor-j ; 2 \ell-1 \mid u\right)$ for $j \in \mathbb{Z} \cap\left[\left\lfloor\frac{m}{2}\right\rfloor+2, m+\left\lfloor\frac{m}{2}\right\rfloor+\right.$ 1]. In addition, it is also appropriate to enlist the differentiated Bessel moments in (1.5)-(1.6), and define $\mathcal{F}_{m, j}^{\ell}(u)$ by updating each occurrence of $\widetilde{\mathbf{I K M}}(\cdot, \cdot ; 2 \ell-1 \mid u)[\operatorname{resp}$. I $\widetilde{\mathbf{K}} \mathbf{M}(\cdot, \cdot ; 2 \ell-1 \mid u)]$ in $\mathcal{F}_{m, j}^{\ell}(u)$ with ÍKM $(\cdot, \cdot ; 2 \ell-1 \mid u)[$ resp. IḰMM $(\cdot, \cdot ; 2 \ell-1 \mid u)]$.

2.2. Block diagonalization of on-shell Wrońskian matrices. Before moving on, we need to reduce the higher-order derivatives in our formulation of Wrońskian matrices into off-shell Bessel moments (1.3)-11.4) and differentiated Bessel moments (1.5)-(1.6).

As in [49, §4.1], we apply the Bessel differential operator iteratively, to derive

$$
\begin{aligned}
& \frac{\mathcal{F}_{m, j}^{\ell}(u)}{4^{\ell-1}}=\left(u D^{2}+D^{1}\right)^{\ell-1} \mathcal{F}_{m, j}(u), \\
& \frac{\dot{\mathcal{F}}_{m, j}^{\ell}(u)}{4^{\ell-1} \sqrt{u}}=2 D^{1}\left(u D^{2}+D^{1}\right)^{\ell-1} \mathcal{F}_{m, j}(u) .
\end{aligned}
$$

In the next lemma, we will spell out the differential operators $\left(u D^{2}+D^{1}\right)^{\ell-1}$ and $D^{1}\left(u D^{2}+D^{1}\right)^{\ell-1}$ as linear differential operators with polynomial coefficients. 
Lemma 2.5 (Iterations of Bessel differential operators). For $n \in \mathbb{Z}_{\geq 0}$, we have

$$
\begin{aligned}
\left(u D^{2}+D^{1}\right)^{n} & =\sum_{m=0}^{n} \frac{n !}{m !}\left(\begin{array}{c}
n \\
m
\end{array}\right) u^{m} D^{n+m}, \\
D^{1}\left(u D^{2}+D^{1}\right)^{n} & =\sum_{m=0}^{n} \frac{n !}{m !}\left(\begin{array}{c}
n+1 \\
m+1
\end{array}\right) u^{m} D^{n+m+1} .
\end{aligned}
$$

Proof. It is clear that (2.17) is valid for $n=0$. Suppose that it holds for $n=N \in \mathbb{Z}_{\geq 0}$, then

$$
\begin{aligned}
& \left(u D^{2}+D^{1}\right)^{N+1} f(u)=\left(u D^{2}+D^{1}\right) \sum_{m=0}^{N} \frac{N !}{m !}\left(\begin{array}{l}
N \\
m
\end{array}\right) u^{m} D^{N+m} f(u) \\
= & \sum_{m=0}^{N} \frac{N !}{m !}\left(\begin{array}{l}
N \\
m
\end{array}\right) u^{m-1}\left[m^{2} D^{0}+(2 m+1) u D^{1}+u^{2} D^{2}\right] D^{N+m} f(u) \\
= & u^{N+1} D^{2 N} f(u)+\sum_{m=0}^{N}\left[(m+1)^{2} \frac{N !}{(m+1) !}\left(\begin{array}{c}
N \\
m+1
\end{array}\right)+(2 m+1) \frac{N !}{m !}\left(\begin{array}{l}
N \\
m
\end{array}\right)\right. \\
& \left.+\frac{N !}{(m-1) !}\left(\begin{array}{c}
N \\
m-1
\end{array}\right)\right] u^{m} D^{N+1+m} f(u) .
\end{aligned}
$$

Since the last bracketed expression in equal to $\frac{(N+1) !}{m !}\left(\begin{array}{c}N+1 \\ m\end{array}\right)$, we know that (2.17) also holds for $n=N+1$.

Now that we have verified (2.17) by induction, we may differentiate its right-hand side termwise, to obtain

$$
\begin{aligned}
& D^{1}\left(u D^{2}+D^{1}\right)^{n} f(u)=D^{1} \sum_{m=0}^{n} \frac{n !}{m !}\left(\begin{array}{c}
n \\
m
\end{array}\right) u^{m} D^{n+m} f(u) \\
= & \sum_{m=0}^{n} \frac{n !}{m !}\left(\begin{array}{c}
n \\
m
\end{array}\right) u^{m-1}\left(m D^{0}+u D^{1}\right) D^{n+m} f(u) \\
= & \sum_{m=0}^{n}\left[(m+1) \frac{n !}{(m+1) !}\left(\begin{array}{c}
n \\
m+1
\end{array}\right)+\frac{n !}{m !}\left(\begin{array}{c}
n \\
m
\end{array}\right)\right] u^{m} D^{n+m+1} f(u) .
\end{aligned}
$$

Here, the last bracketed expression can be identified with $\frac{n !}{m !}\left(\begin{array}{c}n+1 \\ m+1\end{array}\right)$, hence our claim.

Proposition 2.6 (Bessel moment representations of Wrońskian matrices). We have

$$
\begin{aligned}
& \left(\mathcal{F}_{m, j}^{1}(u),(-1)^{2-1} \mathcal{F}_{m, j}^{2}(u), \ldots,(-1)^{\left.\frac{m+1}{2}\right\rfloor-1} \mathcal{F}_{m, j}^{\left\lfloor\frac{m+1}{2}\right\rfloor}(u),\right. \\
& \sqrt{u} \mathcal{F}_{m, j}^{1}(u),(-1)^{2-1} \sqrt{u} \mathcal{F}_{m, j}^{2}(u), \ldots,(-1)^{k-2}{\left.\sqrt{u} \mathcal{F}_{m, j}^{\left\lfloor\frac{m}{2}\right\rfloor}(u)\right)^{\mathrm{T}}} \boldsymbol{\beta}_{m}(u)\left(D^{0} \mathcal{F}_{m, j}(u), \ldots, D^{m-1} \mathcal{F}_{m, j}(u)\right)^{\mathrm{T}},
\end{aligned}
$$

where the $(a, b)$-th entries of the square matrix $\boldsymbol{\beta}_{m}(u) \in \mathbb{Z}[u]^{m \times m}$ are given by

$$
\left(\boldsymbol{\beta}_{m}(u)\right)_{a, b}= \begin{cases}(-4)^{a-1} \frac{(a-1) !}{(b-a) !}\left(\begin{array}{c}
a-1 \\
b-a
\end{array}\right) u^{b-a}, & a \in \mathbb{Z} \cap\left[1,\left\lfloor\frac{m+1}{2}\right\rfloor\right], \\
(-4)^{a-\left\lfloor\frac{m+1}{2}\right\rfloor-1} \frac{2\left(a-\left\lfloor\frac{m+1}{2}\right\rfloor-1\right) !}{\left(b-a+\left\lfloor\frac{m+1}{2}\right\rfloor-1\right) !}\left(\begin{array}{c}
a-\left\lfloor\frac{m+1}{2}\right\rfloor \\
b-a+\left\lfloor\frac{m+1}{2}\right\rfloor
\end{array}\right) u^{b-a+\left\lfloor\frac{m+1}{2}\right\rfloor,} & a \in \mathbb{Z} \cap\left[\left\lfloor\frac{m+1}{2}\right\rfloor+1, m\right] .\end{cases}
$$

Here, it is understood that $\left(\begin{array}{l}N \\ M\end{array}\right)=0$ and $\frac{1}{(-N-1) !}=0$ if $N \in \mathbb{Z}_{\geq 0}$ and $M \in \mathbb{Z} \backslash[0, N]$.

Proof. This is essentially a transcription of the results from Lemma 2.5. [Note: we have abbreviated $\boldsymbol{\beta}_{m}(1)$ as $\boldsymbol{\beta}_{m}$ in (1.18), by slight abuse of notation.]

Remark It is not hard to show, through elementary manipulations of determinants, that $\left|\operatorname{det} \boldsymbol{\beta}_{m}(u)\right|=$ $2^{m(m-1) / 2}|u|^{\left\lfloor m^{2} / 4\right\rfloor}$. Therefore, the matrix $\boldsymbol{\beta}_{m}(u)$ is invertible when $u \neq 0$. 
As we may recall from [49, §4], upon suitable column manipulations, some elements of the matrix $\boldsymbol{\beta}_{m}(u) \mathbf{W}_{m}(u)$ are expressible as on-shell Bessel moments when $u \rightarrow 1^{-}$. Such reductions have led us to factorizations of Wrońskian determinants into products of Broadhurst-Mellit determinants [49, §4]. In the next proposition, we transfer our earlier results onto the matrix $\boldsymbol{\beta}_{m}(u) \mathbf{W}_{m}(u)$ explicitly.

Proposition 2.7 (Block tridiagonalization of Wrońskian matrices). For $k \in \mathbb{Z}_{>0}$, define $\boldsymbol{\alpha}_{k} \in \mathbb{Z}^{(2 k-1) \times(2 k-1)}$ as

$$
\left(\boldsymbol{\alpha}_{k}\right)_{a, b}:= \begin{cases}\delta_{a, b}-\delta_{a+k-1, b}, & a \in \mathbb{Z} \cap[2, k] ; \\ \delta_{a, b}, & a \in \mathbb{Z} \cap(\{1\} \cup[k+1,2 k-1]),\end{cases}
$$

and $\boldsymbol{\psi}_{k} \in \mathbb{Z}^{2 k \times(2 k-1)}, \boldsymbol{\varrho}_{k} \in \mathbb{Z}^{(2 k-1) \times 2 k}$ as

$$
\begin{aligned}
& \left(\boldsymbol{\Psi}_{k}\right)_{a, b}:= \begin{cases}\delta_{a, b}\left(1-\delta_{a, k+1}\right), & a \in \mathbb{Z} \cap[1, k+1] ; \\
\delta_{a, b+1}, & a \in \mathbb{Z} \cap[k, 2 k],\end{cases} \\
& \left(\varrho_{k}\right)_{a, b}:=\delta_{a, b},
\end{aligned}
$$

so that the $(k+1)$-st column and the bottom row of $\mathbf{X} \in \mathbb{R}^{2 k \times 2 k}$ are dropped to form $\boldsymbol{\varrho}_{k} \mathbf{X} \boldsymbol{\psi}_{k} \in \mathbb{R}^{(2 k-1) \times(2 k-1)}$. For period matrices $\mathbf{P}_{m}:=\left((-1)^{b-1} \pi^{a-\frac{m+3}{2}} \mathbf{I K M}(a, m+2-a ; 2 b-1)\right)_{1 \leq a, b \leq\left\lfloor\frac{m+1}{2}\right\rfloor}$ of all sizes, we have

$$
\boldsymbol{\beta}_{2 k-1}(1) \mathbf{W}_{2 k-1}(1) \boldsymbol{\alpha}_{k}=\left(\begin{array}{ll}
\mathbf{P}_{2 k-1}^{\mathrm{T}} & \\
\mathbf{P}_{2 k-1}^{\mathrm{T}} & -\mathbf{P}_{2 k-3}^{\mathrm{T}}
\end{array}\right)
$$

and

$$
\boldsymbol{\varrho}_{k} \boldsymbol{\beta}_{2 k}(1) \lim _{u \rightarrow 1^{-}} \mathbf{W}_{2 k}(u) \boldsymbol{\Psi}_{k} \boldsymbol{\alpha}_{k}=\left(\begin{array}{ll}
\mathbf{P}_{2 k}^{\mathrm{T}} & \\
\hat{\mathbf{P}}_{2 k}^{\mathrm{T}} & -\mathbf{P}_{2 k-2}^{\mathrm{T}}
\end{array}\right),
$$

where the unwritten blocks in the top-right positions are filled with zeros, and $\mathbf{\mathbf { P }}_{m}:=$ $\left((-1)^{b-1} \mathcal{F}_{m, a}^{b}(1)\right)_{1 \leq a \leq\left\lfloor\frac{m+1}{2}\right\rfloor, 1 \leq b \leq\left\lfloor\frac{m-1}{2}\right\rfloor}$.

Proof. Multiplying $\boldsymbol{\alpha}_{k}$ to the right of a $(2 k-1) \times(2 k-1)$ matrix, we are effectively subtracting its 2 nd to $k$-th columns from its last $(k-1)$ columns. By definition, we have $\mathcal{F}_{m, j}^{\ell}(1)-\mathcal{F}_{m, j+\left\lfloor\frac{m+1}{2}\right\rfloor-1}^{\ell}(1)=0, \forall j \in \mathbb{Z} \cap$ $\left[2,\left\lfloor\frac{m+1}{2}\right\rfloor\right]$, accounting for the top-right blocks with vanishing entries in (2.26) and (2.27). Meanwhile, the Wrońskian relation $I_{1}(t) K_{0}(t)+I_{0}(t) K_{1}(t)=\frac{1}{t}$ leads us to an identity $\dot{\mathcal{F}}_{m, j}^{\ell}(1)-\dot{\mathcal{F}}_{m, j+\left\lfloor\frac{m+1}{2}\right\rfloor-1}^{\ell}(1)=$ $-\mathcal{F}_{m-2, j-1}^{\ell}(1), \forall j \in \mathbb{Z} \cap\left[2,\left\lfloor\frac{m+1}{2}\right\rfloor\right]$, accounting for the bottom-right blocks in (2.26) and (2.27). It is clear that the bottom-left blocks $\dot{\mathbf{P}}_{2 k-1}^{\mathrm{T}}$ and $\mathbf{\mathbf { P }}_{2 k}^{\mathrm{T}}$ originate from the intact entries in $\boldsymbol{\beta}_{2 k-1}(1) \mathbf{W}_{2 k-1}(1)$ and $\boldsymbol{\varrho}_{k} \boldsymbol{\beta}_{2 k}(1) \lim _{u \rightarrow 1^{-}} \mathbf{W}_{2 k}(u) \boldsymbol{\psi}_{k}$.

The configurations in (2.26) and (2.27) were precisely the forms that we prepared [49, Propositions 4.3 and 4.6] for our proof of the Broadhurst-Mellit determinant formulae. To get ready for later developments in $\$ 4.1$ of the current article, we need to go one step further, eliminating the bottom-left blocks $\hat{\mathbf{P}}_{2 k-1}^{\mathrm{T}}$ and $\mathbf{P}_{2 k}^{\mathrm{T}}$ in the next proposition.

Proposition 2.8 (Block diagonalization of Wrońskian matrices). Define square matrices $\boldsymbol{\vartheta}_{m}, \boldsymbol{\varphi}_{m} \in$ $\mathbb{Q}^{\left(2\left\lfloor\frac{m+1}{2}\right\rfloor-1\right) \times\left(2\left\lfloor\frac{m+1}{2}\right\rfloor-1\right)}$ as follows:

$$
\begin{aligned}
\left(\boldsymbol{\vartheta}_{m}\right)_{a, b} & := \begin{cases}\delta_{a, b}, & a \in \mathbb{Z} \cap\left[1,\left\lfloor\frac{m+1}{2}\right\rfloor\right], \\
\delta_{a, b}+\frac{2\left(a-\left\lfloor\frac{m+1}{2}\right\rfloor\right)}{m+2} \delta_{a-\left\lfloor\frac{m+1}{2}\right\rfloor, b}, & a \in \mathbb{Z} \cap\left[\left\lfloor\frac{m+1}{2}\right\rfloor+1,2\left\lfloor\frac{m+1}{2}\right\rfloor-1\right] ;\end{cases} \\
\left(\boldsymbol{\varphi}_{m}\right)_{a, b}: & = \begin{cases}\delta_{a, b}, & a \in \mathbb{Z} \cap\left[1,\left\lfloor\frac{m+1}{2}\right\rfloor\right], \\
\delta_{a, b}+\left(1-\frac{b}{m+2}\right) \delta_{a-\left\lfloor\frac{m+1}{2}\right\rfloor+1, b}, & a \in \mathbb{Z} \cap\left[\left\lfloor\frac{m+1}{2}\right\rfloor+1,2\left\lfloor\frac{m+1}{2}\right\rfloor-1\right] .\end{cases}
\end{aligned}
$$


For $k \in \mathbb{Z}_{>1}$, we have

$$
\boldsymbol{\vartheta}_{2 k-1} \boldsymbol{\beta}_{2 k-1}(1) \mathbf{W}_{2 k-1}(1) \boldsymbol{\alpha}_{k} \boldsymbol{\varphi}_{2 k-1}=\left(\begin{array}{ll}
\mathbf{P}_{2 k-1}^{\mathrm{T}} & \\
& -\mathbf{P}_{2 k-3}^{\mathrm{T}}
\end{array}\right)
$$

and

$$
\boldsymbol{\vartheta}_{2 k} \boldsymbol{\varrho}_{k} \boldsymbol{\beta}_{2 k}(1) \lim _{u \rightarrow 1^{-}} \mathbf{W}_{2 k}(u) \boldsymbol{\psi}_{k} \boldsymbol{\alpha}_{k} \boldsymbol{\varphi}_{2 k}=\left(\begin{array}{ll}
\mathbf{P}_{2 k}^{\mathrm{T}} & \\
& -\mathbf{P}_{2 k-2}^{\mathrm{T}}
\end{array}\right)
$$

Proof. Clearly, it would suffice to check that the matrix elements of $\dot{\mathbf{P}}_{m}$ can be alternatively represented by

$$
\left(\dot{\mathbf{P}}_{m}\right)_{a, b}:= \begin{cases}(-1)^{b} \frac{2 b}{m+2} \mathcal{F}_{m, 1}^{b}(1), & a=1, \\ (-1)^{b}\left[\frac{2 b}{m+2} \mathcal{F}_{m, a}^{b}(1)-\left(1-\frac{a}{m+2}\right) \mathcal{F}_{m-2, a-1}^{b}(1)\right], & a \in \mathbb{Z} \cap\left[2,\left\lfloor\frac{m+1}{2}\right\rfloor\right] ;\end{cases}
$$

Integrating by parts, we have

$$
\begin{aligned}
\mathcal{F}_{m, 1}^{\ell}(1) & :=\frac{1}{m} \int_{0}^{\infty} I_{1}(t)\left[K_{0}(t)\right]^{m-1} t^{2 \ell} \mathrm{d} t-\frac{m-1}{m} \int_{0}^{\infty} K_{1}(t) I_{0}(t)\left[K_{0}(t)\right]^{m-2} t^{2 \ell-1} \mathrm{~d} t \\
& =\frac{1}{m} \int_{0}^{\infty} t^{2 \ell} \frac{\mathrm{d}}{\mathrm{d} t}\left\{I_{0}(t)\left[K_{0}(t)\right]^{m-1}\right\} \mathrm{d} t=-\frac{2 \ell}{m} \mathcal{F}_{m, 1}^{\ell}(1) .
\end{aligned}
$$

Likewise, for $j \in \mathbb{Z} \cap\left[2,\left\lfloor\frac{m+1}{2}\right\rfloor\right]$, we have

$$
\begin{aligned}
\dot{\mathcal{F}}_{m, j}^{\ell}(1) & :=\int_{0}^{\infty} I_{1}(t)\left[I_{0}(t)\right]^{j-1}\left[K_{0}(t)\right]^{m-j} t^{2 \ell} \mathrm{d} t=\frac{1}{j} \int_{0}^{\infty}\left[K_{0}(t)\right]^{m-j} t^{2 \ell} \frac{\mathrm{d}}{\mathrm{d} t}\left[I_{0}(t)\right]^{j} \mathrm{~d} t \\
& =-\frac{2 \ell}{j} \mathcal{F}_{m, j}^{\ell}(1)+\frac{m-j}{j} \int_{0}^{\infty} K_{1}(t)\left[I_{0}(t)\right]^{j}\left[K_{0}(t)\right]^{m-j-1} t^{2 \ell} \mathrm{d} t \\
& =-\frac{2 \ell}{j} \mathcal{F}_{m, j}^{\ell}(1)+\frac{m-j}{j}\left[\mathcal{F}_{m-2, j-1}^{\ell}(1)-\dot{\mathcal{F}}_{m, j}^{\ell}(1)\right] .
\end{aligned}
$$

This can be rearranged into

$$
\dot{\mathcal{F}}_{m, j}^{\ell}(1)=\left(1-\frac{j}{m}\right) \mathcal{F}_{m-2, j-1}^{\ell}(1)-\frac{2 \ell}{m} \mathcal{F}_{m, j}^{\ell}(1)
$$

which completes our task.

2.3. Sum rules for on-shell and off-shell Bessel moments. When $u=\left(2 j-\frac{1+(-1)^{m}}{2}\right)^{2}, j \in \mathbb{Z} \cap[1,\lfloor m / 2\rfloor+$ 1], certain functions among $\mathcal{F}_{m, j}^{\ell}(u), \mathcal{F}_{m, j}^{\ell}(u)$ for $j \in \mathbb{Z} \cap[1, m+\lfloor m / 2\rfloor+1]$ assume finite values, and are $\mathbb{Q}$-linearly dependent. We refer to such $\mathbb{Q}$-linear dependence relations (together with their modest generalizations) as sum rules for Bessel moments at thresholds. Here, we choose the word "threshold" to acknowledge the fact that certain functions among $\mathcal{F}_{m, j}^{\ell}(u), \dot{\mathcal{F}}_{m, j}^{\ell}(u)$ for $j \in \mathbb{Z} \cap[1, m+\lfloor m / 2\rfloor+1]$ go unbounded as $u \rightarrow\left(2 j-\frac{1+(-1)^{m}}{2}\right)^{2} \pm 0^{+}, j \in \mathbb{Z} \cap[1,\lfloor m / 2\rfloor+1]$. (See Lemma 3.4 for quantitative characterization of such divergent behavior near the threshold.) 
These sum rules with rational coefficients will be vital to the analysis of Wrońskian cofactors in $\$ 3.2$, which revolves around the identity

$$
\left(\begin{array}{c}
(-1)^{m+1} W\left[\widehat{\mathcal{F}_{m, 1}(u)}, \ldots, \mathcal{F}_{m, r}(u), \ldots, \mathcal{F}_{m, m}(u)\right] \\
\vdots \\
(-1)^{m+r} W\left[\mathcal{F}_{m, 1}(u), \ldots, \widehat{\mathcal{F}}_{m, r}(u), \ldots, \mathcal{F}_{m, m}(u)\right] \\
\vdots \\
W\left[\mathcal{F}_{m, 1}(u), \ldots, \mathcal{F}_{m, r}(u), \ldots, \widehat{\mathcal{F}_{m, m}(u)}\right]
\end{array}\right)=\frac{\Lambda_{m}}{\left|\mathcal{L}_{m}(u)\right|^{\frac{m-2}{2}}} \mathbf{S}_{m}\left(\begin{array}{c}
\mathcal{F}_{m, 1}(u) \\
\vdots \\
\mathcal{F}_{m, r}(u) \\
\vdots \\
\mathcal{F}_{m, m}(u)
\end{array}\right)
$$

Here on the left-hand side, a caret indicates removal of the term underneath while evaluating the Wrońskian determinant of $(m-1)$ functions; on the right-hand side, we have [see (1.9)]

$$
\Lambda_{m}:=W_{m}(u)\left|\mathcal{L}_{m}(u)\right|^{m / 2}=\frac{m+1}{m+2} \frac{(-1)^{\lfloor m / 4\rfloor}}{2^{m(m-1) / 2}} \frac{[(m+1) !]^{m}}{\prod_{n=1}^{m+1} n^{n}}
$$

and $\mathbf{S}_{m} \in \mathbb{Q}^{m \times m}$ is a matrix filled with rational numbers. At the end of this section, we will illustrate the usefulness of $\mathbb{Q}$-linear sum rules with an explicit computation of $\mathbf{S}_{3}$ (Corollary 2.11), while deferring the treatment of generic $\mathbf{S}_{m}$ until the next section.

To facilitate future discussion of sum rules, we introduce the following formal notations for $m \in$ $\mathbb{Z}_{>0}, s \in \mathbb{Z} \cap\left[1,\left\lfloor\frac{m}{2}\right\rfloor+1\right]: 5$

$$
\begin{aligned}
\mathcal{I}_{m, s}^{\ell}(u):= & (m+2) \mathcal{F}_{m, 1}^{\ell}(u)+\sum_{j=1}^{\left\lfloor\frac{\lfloor m / 2\rfloor+1-s}{2}\right\rfloor}(-1)^{j}\left(\begin{array}{c}
\left\lfloor\frac{m}{2}\right\rfloor+1-s \\
2 j
\end{array}\right) \mathcal{F}_{m, 2 j+1}^{\ell}(u) \\
& -(-1)^{m} i \sum_{j=0}^{\left\lfloor\frac{\lfloor m / 2\rfloor-s}{2}\right\rfloor}(-1)^{j}\left(\begin{array}{c}
\left\lfloor\frac{m}{2}\right\rfloor+1-s \\
2 j+1
\end{array}\right) \mathcal{F}_{m, 2 j+2}^{\ell}(u), \\
\mathcal{K}_{m, s}^{\ell}(u):= & -\sum_{j=1}^{\left.\frac{\lfloor(m+1) / 2\rfloor-1+s}{2}\right\rfloor}(-1)^{j}\left[\left(\begin{array}{c}
\left\lfloor\frac{m}{2}\right\rfloor+1-s \\
2 j+1
\end{array}\right)+\left(\begin{array}{c}
\left\lfloor\frac{m+1}{2}\right\rfloor+s \\
2 j+1
\end{array}\right)\right] \mathcal{F}_{m, 2 j+\left\lfloor\frac{m}{2}\right\rfloor+1}^{\ell}(u) \\
& -(-1)^{m} i \sum_{j=1}^{\left\lfloor\frac{\lfloor(m+1) / 2\rfloor+s}{2}\right\rfloor}(-1)^{j}\left[\left(\begin{array}{c}
\left.\frac{m}{2}\right\rfloor+1-s \\
2 j
\end{array}\right)-\left(\begin{array}{c}
\left.\frac{m+1}{2}\right\rfloor+s \\
2 j
\end{array}\right)\right] \mathcal{F}_{m, 2 j+\left\lfloor\frac{m}{2}\right\rfloor}^{\ell}(u) .
\end{aligned}
$$

The domains of definition for the real-valued functions $\operatorname{Re} \mathcal{I}_{m, s}^{\ell}(u), \operatorname{Im} \mathcal{I}_{m, s}^{\ell}(u)$ and so forth will be clear from context 6 We also write $\dot{\mathcal{I}}_{m, s}^{\ell}(u)\left[\operatorname{resp} . \mathcal{K}_{m, s}^{\ell}(u)\right.$ ] for expressions that trade $\mathcal{F}$ in $\mathcal{I}_{m, s}^{\ell}(u)$ [resp. $\left.\mathcal{K}_{m, s}^{\ell}(u)\right]$ with $\dot{\mathcal{F}}$, and set $\mathrm{F}_{k, s}^{\ell}=\mathcal{I}_{2 k-1, s}^{\ell}-\mathcal{K}_{2 k-1, s}^{\ell}, \dot{F}_{k, s}^{\ell}=\dot{\mathcal{I}}_{2 k-1, s}^{\ell}-\dot{\mathcal{K}}_{2 k-1, s}^{\ell}, \mathrm{G}_{k, s}^{\ell}=\mathcal{I}_{2 k, s}^{\ell}-\mathcal{K}_{2 k, s}^{\ell}, \dot{\mathrm{G}}_{k, s}^{\ell}=$ $\dot{\mathcal{I}}_{2 k, s}^{\ell}-\mathcal{K}_{2 k, s}^{\ell}$.

Proposition 2.9 (Sum rules at thresholds). In what follows, assume that $k \in \mathbb{Z}_{>1}$.

(a) For $s \in \mathbb{Z} \cap[1, k], \ell \in \mathbb{Z} \cap\left[1,\left\lfloor\frac{k}{2}\right\rfloor\right]$, we have $\mathrm{F}_{k, s}^{\ell}\left((2 s)^{2}\right)=0$; for $s \in \mathbb{Z} \cap[1, k], \ell \in \mathbb{Z} \cap\left[1,\left\lfloor\frac{k-1}{2}\right\rfloor\right]$, we have $\hat{\mathrm{F}}_{k, s}^{\ell}\left((2 s)^{2}\right)=0$.

(b) For $s \in \mathbb{Z} \cap[1, k+1], \ell \in \mathbb{Z} \cap\left[1,\left\lfloor\frac{k}{2}\right\rfloor\right]$, we have $\mathrm{G}_{k, s}^{\ell}\left((2 s-1)^{2}\right)=0$; for $s \in \mathbb{Z} \cap[1, k+1], \ell \in \mathbb{Z} \cap$ $\left[1,\left\lfloor\frac{k-1}{2}\right\rfloor\right]$, we have $\mathrm{G}_{k, s}^{\ell}\left((2 s-1)^{2}\right)=0$.

\footnotetext{
${ }^{5}$ It is understood that expressions like $\sum_{j=1}^{0}(\cdots)$ are empty sums, hence vanishing.

${ }^{6}$ Note that $\operatorname{Re} \mathcal{I}_{m, s}^{\ell}(u)$ and $\operatorname{Im} \mathcal{I}_{m, s}^{\ell}(u)$ do not necessarily share their domains of definition, neither do $\operatorname{Re} \mathcal{K}_{m, s}^{\ell}(u)$ and $\operatorname{Im} \mathcal{K}_{m, s}^{\ell}(u)$. In view of this, the notations $\mathcal{I}_{m, s}^{\ell}(u), \mathcal{K}_{m, s}^{\ell}(u)$ and so forth are convenient short-hands, instead of complex-valued functions in the usual sense.
} 
Proof. As in [53, §2.1, §3] and [52, Remark after Lemma 3.2], we will prove these sum rules by studying contour integrals involving the cylindrical Hankel functions $H_{0}^{(1)}(z), H_{0}^{(2)}(z), H_{1}^{(1)}(z), H_{1}^{(2)}(z)$ for $z \in \mathbb{C} \backslash$ $(-\infty, 0]$, whose asymptotic behavior reads

$$
\left\{\begin{array}{l}
H_{n}^{(1)}(z)=\sqrt{\frac{2}{\pi z}} e^{+i\left(z-\frac{n \pi}{2}-\frac{\pi}{4}\right)}\left[1+O\left(\frac{1}{|z|}\right)\right] \\
H_{n}^{(2)}(z)=\sqrt{\frac{2}{\pi z}} e^{-i\left(z-\frac{n \pi}{2}-\frac{\pi}{4}\right)}\left[1+O\left(\frac{1}{|z|}\right)\right]
\end{array}\right.
$$

for $n \in\{0,1\}$, as $|z| \rightarrow \infty,-\pi<\arg z<\pi$. Furthermore, these Hankel functions are related to modified Bessel functions $I_{0}(t), K_{0}(t), I_{1}(t), K_{1}(t)$ for $t>0$ in the following manner:

$$
\left\{\begin{array} { l } 
{ H _ { n } ^ { ( 1 ) } ( + i t ) = - \frac { 2 i ^ { 1 - n } } { \pi } K _ { n } ( t ) } \\
{ H _ { n } ^ { ( 1 ) } ( - i t ) = \frac { 2 } { i ^ { n } } I _ { n } ( t ) - \frac { 2 i ^ { n + 1 } } { \pi } K _ { n } ( t ) }
\end{array} \text { and } \left\{\begin{array}{l}
H_{n}^{(2)}(+i t)=2 i^{n} I_{n}(t)+\frac{2 i^{1-n}}{\pi} K_{n}(t) \\
H_{n}^{(2)}(-i t)=\frac{2 i^{n+1}}{\pi} K_{n}(t)
\end{array}\right.\right.
$$

where $n \in\{0,1\}$.

(a) Armed with the aforementioned preparations, we use two methods to evaluate the contour integral

$$
\int_{-i \infty}^{i \infty} H_{n}^{(2)}(2 s z)\left[H_{0}^{(1)}(z)\right]^{2 s}\left[H_{0}^{(1)}(z) H_{0}^{(2)}(z)\right]^{k-s} z^{2 \ell+n-1} \mathrm{~d} z
$$

which converges for $k \in \mathbb{Z}_{>1}, s \in \mathbb{Z} \cap[1, k], n \in\{0,1\}, \ell \in \mathbb{Z} \cap\left[1,\left\lfloor\frac{k-n}{2}\right\rfloor\right]$. First, by the asymptotic behavior of cylindrical Hankel functions in (2.40), we can close the contour to the right, and show that the integral vanishes. Second, with the aid of 2.41), we may recognize the contour integral as a constant multiple of

$$
\begin{aligned}
& \int_{0}^{\infty}\left[i^{n} \pi I_{n}(2 s t)+i^{1-n} K_{n}(2 s t)\right]\left[-i K_{0}(t)\right]^{k+s}\left[\pi I_{0}(t)+i K_{0}(t)\right]^{k-s} t^{2 \ell+n-1} \mathrm{~d} t \\
& -(-1)^{n} \int_{0}^{\infty} i^{n+1} K_{n}(2 s t)\left[\pi I_{0}(t)-i K_{0}(t)\right]^{k+s}\left[i K_{0}(t)\right]^{k-s} t^{2 \ell+n-1} \mathrm{~d} t
\end{aligned}
$$

For $n=0$, the last vanishing integral reduces to a sum rule

$$
\begin{aligned}
& \int_{0}^{\infty}\left[\pi I_{0}(2 s t)+i K_{0}(2 s t)\right] \sum_{j=0}^{k-s}\left(\begin{array}{c}
k-s \\
j
\end{array}\right)\left[\pi I_{0}(t)\right]^{j}\left[i K_{0}(t)\right]^{2 k-j} t^{2 \ell+n-1} \mathrm{~d} t \\
& -\int_{0}^{\infty} i K_{0}(2 s t) \sum_{j=0}^{k+s}\left(\begin{array}{c}
k+s \\
j
\end{array}\right)\left[\pi I_{0}(t)\right]^{j}\left[-i K_{0}(t)\right]^{2 k-j} t^{2 \ell+n-1} \mathrm{~d} t=0,
\end{aligned}
$$

which is equivalent to $\mathrm{F}_{k, s}^{\ell}\left((2 s)^{2}\right)=0$. For $n=1$, we can deduce $\tilde{F}_{k, s}^{\ell}\left((2 s)^{2}\right)=0$ instead.

(b) When $k \in \mathbb{Z}_{>1}, s \in \mathbb{Z} \cap[1, k+1], n \in\{0,1\}, \ell \in \mathbb{Z} \cap\left[1,\left\lfloor\frac{k-n}{2}\right\rfloor\right]$, we can evaluate

$$
\int_{-i \infty}^{i \infty} H_{n}^{(2)}((2 s-1) z)\left[H_{0}^{(1)}(z)\right]^{2 s-1}\left[H_{0}^{(1)}(z) H_{0}^{(2)}(z)\right]^{k+1-s} z^{2 \ell+n-1} \mathrm{~d} z=0
$$

by closing the contour to the right, while noting that the integrand has $O\left(|z|^{-2}\right)$ behavior for large $|z|$. This can be reinterpreted into the sum rules $\mathrm{G}_{k, s}^{\ell}\left((2 s-1)^{2}\right)=0$ and $\mathrm{G}_{k, s}^{\ell}\left((2 s-1)^{2}\right)=0$.

The sum rules in the last proposition will be essential to local analysis 7 in $\$ 3.2$, especially the determination of the blocks $\mathbf{S}_{m}^{A}$ [as given in (1.13)] and $\mathbf{S}_{m}^{B}=(-1)^{m+1}\left(\mathbf{S}_{m}^{C}\right)^{\mathrm{T}}$ [as given in (1.14)] for the partitioned matrix $\mathbf{S}_{m}=\left(\begin{array}{ll}\mathbf{S}_{m}^{A} & \mathbf{S}_{m}^{B} \\ \mathbf{S}_{m}^{C} & \mathbf{S}_{m}^{D}\end{array}\right)$ that fits into (2.36). To compute the block $\mathbf{S}_{m}^{D}$, and to complete the proof of Theorem 1.1 in $\$ 3.3$, we will need to investigate another threshold $u \rightarrow 0^{+}$in the next proposition.

\footnotetext{
${ }^{7}$ Here, by "local analysis", we refer to asymptotic expansions for both sides of (2.36) (with appropriate real-analytic continuations if necessary) when $u$ approaches a threshold. Corollary 2.11 illustrates such local analysis in the case where $m=3$.
} 
In what follows, we define rational numbers $\mathrm{H}_{k, n}$ for $k, n \in \mathbb{Z}_{>0}$ through the following asymptotic expansion (cf. [53, (66)]):

$$
h_{k, N}(z):=\left[\frac{\pi}{2} H_{0}^{(1)}(z) H_{0}^{(2)}(z)\right]^{k}-\sum_{n=1}^{N} \frac{\mathrm{H}_{k, n}}{z^{2 n+k-2}}=O\left(\frac{1}{|z|^{2 N+k}}\right),
$$

where $k, N \in \mathbb{Z}_{>0}$. These rational numbers also turn up in the relations for generalized Crandall numbers (cf. [53, (65)]):

$$
(-1)^{n+1}\left(\frac{\pi}{2}\right)^{k} \mathrm{H}_{k, n}=\int_{0}^{\infty} \frac{\left[\pi I_{0}(t)+i K_{0}(t)\right]^{k}-\left[\pi I_{0}(t)-i K_{0}(t)\right]^{k}}{\pi i}\left[K_{0}(t)\right]^{k} t^{2 n+k-3} \mathrm{~d} t .
$$

By the convention that empty sums must vanish, we retroactively define $h_{k, 0}(z)=\left[\frac{\pi}{2} H_{0}^{(1)}(z) H_{0}^{(2)}(z)\right]^{k}$ and $\mathrm{H}_{k, 0}=0$. Furthermore, we introduce a function

$$
\mathrm{L}_{k}(u, t):=\sum_{n=1}^{\infty} \frac{\pi^{1-k} u^{n+\frac{k}{2}-1} t^{2 n+k-3}}{2^{2 n-1}\left[\Gamma\left(n+\frac{k}{2}\right)\right]^{2}} \equiv \frac{u^{k / 2} t^{k-1}}{2 \pi^{k-1}\left[\Gamma\left(\frac{k}{2}+1\right)\right]^{2}}{ }_{1} F_{2}\left(\begin{array}{c|c}
1 \\
\frac{k}{2}+1, \frac{k}{2}+1
\end{array} \mid \frac{u t^{2}}{4}\right) .
$$

For each fixed $u \in(0,1)$, we have $\mathrm{L}_{k}(u, t)=O(1)$ as $t \rightarrow 0^{+}$, and $\mathrm{L}_{k}(u, t)=O\left(e^{\sqrt{u} t} / \sqrt{t}\right)$ as $t \rightarrow \infty$, so the dominated convergence theorem allows us to conclude that either side of

$$
\int_{0}^{\infty} \frac{\left[\pi I_{0}(t)+i K_{0}(t)\right]^{k}-\left[\pi I_{0}(t)-i K_{0}(t)\right]^{k}}{\pi i}\left[K_{0}(t)\right]^{k} L_{k}(u, t) \mathrm{d} t=\sum_{n=1}^{\infty} \frac{\pi(-1)^{n+1} \mathrm{H}_{k, n} u^{n+\frac{k}{2}-1}}{2^{2 n+k-1}\left[\Gamma\left(n+\frac{k}{2}\right)\right]^{2}}
$$

represents a continuous function in the variable $u \in(0,1)$.

Proposition 2.10 ("Sum rules" at threshold $u \rightarrow 0^{+}$). (a) For $\ell \in \mathbb{Z}_{>0}$, we have 8

$$
\mathcal{F}_{2 k-1,2 k-1}^{\ell}(u)=O\left(\frac{1}{(\sqrt{u})^{\max \{1,2 \ell-k\}}}\right), \sqrt{u} \mathcal{F}_{2 k-1,2 k-1}^{\ell}(u)=O\left(\frac{1}{(\sqrt{u})^{\max \{1,2 \ell-k\}}}\right),
$$

as $u \rightarrow 0^{+}$.

For each $k \in 1+2 \mathbb{Z}_{\geq 0}$ and $\ell \in \mathbb{Z}_{>0}$, we have the following quantitative refinements of (2.50) for $u \in(0,1)$ :

$$
\begin{aligned}
O_{k}^{\ell}(u) & :=(2 k+1) \mathcal{F}_{2 k-1,1}^{\ell}(u)+\sum_{b=2}^{k} \frac{1+(-1)^{b-1}}{2(-1)^{\left\lfloor\frac{b-1}{2}\right\rfloor}}\left(\begin{array}{c}
k \\
b-1
\end{array}\right) \mathcal{F}_{2 k-1, b}^{\ell}(u)+\sum_{b=k+1}^{2 k-1} \frac{1+(-1)^{b-k}}{(-1)^{\left\lfloor\frac{b-k}{2}\right\rfloor}}\left(\begin{array}{c}
k \\
b-k+1
\end{array}\right) \mathcal{F}_{2 k-1, b}^{\ell}(u) \\
& =4^{\ell}\left(u D^{2}+D^{1}\right)^{\ell} \int_{0}^{\infty} \frac{\left[\pi I_{0}(t)+i K_{0}(t)\right]^{k}-\left[\pi I_{0}(t)-i K_{0}(t)\right]^{k}}{(-1)^{\frac{k+1}{2}} 2^{k} \pi i}\left[K_{0}(t)\right]^{k} L_{k}(u, t) \mathrm{d} t ; \\
\hat{O}_{k}^{\ell}(u) & :=(2 k+1) \dot{\mathcal{F}}_{2 k-1,1}^{\ell}(u)+\sum_{b=2}^{k} \frac{1+(-1)^{b-1}}{2(-1)^{\left\lfloor\frac{b-1}{2}\right\rfloor}}\left(\begin{array}{c}
k \\
b-1
\end{array}\right) \dot{\mathcal{F}}_{2 k-1, b}^{\ell}(u)+\sum_{b=k+1}^{2 k-1} \frac{1+(-1)^{b-k}}{(-1)^{\left\lfloor\frac{b-k}{2}\right\rfloor}}\left(\begin{array}{c}
k \\
b-k+1
\end{array}\right) \dot{\mathcal{F}}_{2 k-1, b}^{\ell}(u) \\
& =4^{\ell} \sqrt{u} D^{1}\left(u D^{2}+D^{1}\right)^{\ell} \int_{0}^{\infty} \frac{\left[\pi I_{0}(t)+i K_{0}(t)\right]^{k}-\left[\pi I_{0}(t)-i K_{0}(t)\right]^{k}}{(-1)^{\frac{k+1}{2}} 2^{k-1} \pi i}\left[K_{0}(t)\right]^{k} L_{k}(u, t) \mathrm{d} t .
\end{aligned}
$$

\footnotetext{
${ }^{8}$ The asymptotic behavior of $\mathcal{F}_{2 k-1,2 k-1}^{\ell}(u)$ and $\mathcal{F}_{2 k-1,2 k-1}^{\ell}(u)$ was misstated as $O(1 / \sqrt{u})$ (irrespective of $\ell$ ) in [49, Proposition 4.4]. The corrections here do not affect the proof of [49, Proposition 4.4], the latter of which only requires $\lim _{u \rightarrow 0^{+}} u^{k / 2} \mathcal{F}_{2 k-1,2 k-1}^{\ell}(u) \log u=\lim _{u \rightarrow 0^{+}} u^{k / 2} \sqrt{u}_{2 k-1,2 k-1}^{\ell}(u) \log u=0$ for $\ell \in \mathbb{Z} \cap[1, k-1]$ to work properly.
} 
For each $k \in 2 \mathbb{Z}_{>0}$ and $\ell \in \mathbb{Z}_{>0}$, we have the following quantitative refinements of (2.50) in the $u \rightarrow 0^{+}$regime:

$$
\begin{aligned}
E_{k}^{\ell}(u):= & \sum_{b=k+1}^{2 k-1} \frac{1-(-1)^{b-k}}{(-1)^{\left\lfloor\frac{b-k}{2}\right\rfloor}}\left(\begin{array}{c}
k \\
b-k+1
\end{array}\right) \mathcal{F}_{2 k-1, b}^{\ell}(u)+\frac{2 \log \frac{\sqrt{u}}{2}}{\pi}\left[(2 k+1) \mathcal{F}_{2 k-1,1}^{\ell}(u)+\right. \\
& \left.+\sum_{b=k+1}^{2 k-1} \frac{1+(-1)^{b-k}}{(-1)^{\left\lfloor\frac{b-k}{2}\right\rfloor}}\left(\begin{array}{c}
k \\
b-k+1
\end{array}\right) \mathcal{F}_{2 k-1, b}^{\ell}(u)\right]-\frac{\log ^{2} \frac{\sqrt{u}}{2}}{\pi^{2}} \sum_{b=2}^{k} \frac{1+(-1)^{b}}{(-1)^{\left\lfloor\frac{b}{2}\right\rfloor}}\left(\begin{array}{c}
k \\
b-1
\end{array}\right) \mathcal{F}_{2 k-1, b}^{\ell}(u) \\
= & \mathscr{E}_{k}^{\ell}(u)+\sum_{n \in \mathbb{Z} \cap\left[1, \max \left\{0, \ell-\frac{k}{2}\right\}\right]} \frac{2^{2(\ell-n-k)+1}\left[\left(\ell-n-\frac{k}{2}\right) !\right]^{2}}{(-1)^{n+k / 2} \pi u^{\ell-n+1-\frac{k}{2}}} \mathrm{H}_{k, n}, \\
\dot{E}_{k}^{\ell}(u):= & \sum_{b=k+1}^{2 k-1} \frac{1-(-1)^{b-k}}{(-1)^{\left\lfloor\frac{b-k}{2}\right\rfloor}}\left(\begin{array}{c}
k \\
b-k+1
\end{array}\right) \dot{\mathcal{F}}_{2 k-1, b}^{\ell}(u)+\frac{2 \log \frac{\sqrt{u}}{2}}{\pi}\left[(2 k+1) \dot{\mathcal{F}}_{2 k-1,1}^{\ell}(u)+\right. \\
& \left.+\sum_{b=k+1}^{2 k-1} \frac{1+(-1)^{b-k}}{(-1)^{\left\lfloor\frac{b-k}{2}\right\rfloor}}\left(\begin{array}{c}
k \\
b-k+1
\end{array}\right) \dot{\mathcal{F}}_{2 k-1, b}^{\ell}(u)\right]-\frac{\log ^{2} \frac{\sqrt{u}}{2}}{\pi^{2}} \sum_{b=2}^{k} \frac{1+(-1)^{b}}{(-1)^{\left\lfloor\frac{b}{2}\right\rfloor}}\left(\begin{array}{c}
k \\
b-1
\end{array}\right) \dot{\mathcal{F}}_{2 k-1, b}^{\ell}(u) \\
= & \frac{\mathscr{E}_{k}^{\ell}(u)}{\sqrt{u}+} \sum_{n \in \mathbb{Z} \cap\left[1, \max \left\{0, \ell-\frac{k}{2}\right\}\right]} \frac{4^{\ell-n-k+1}\left(\ell-n-\frac{k}{2}\right) !\left(\ell-n-\frac{k}{2}+1\right) !}{(-1)^{n-1+k / 2} \pi u^{\ell-n+\frac{3}{2}-\frac{k}{2}}} \mathrm{H}_{k, n},
\end{aligned}
$$

where $\mathscr{E}_{k}^{\ell}(u)$ and $\mathscr{E}_{k}^{\ell}(u)$ are holomorphic in a non-void $\mathbb{C}$-neighborhood of $u=0$.

Furthermore, if a function in $\operatorname{span}_{\mathbb{C}}\left\{\mathcal{F}_{2 k-1, b}(u), u \in(0, \varepsilon) \mid b \in \mathbb{Z} \cap(\{1\} \cup[k+1,2 k-1])\right\}$ (where $0<$ $\varepsilon<1$ ) extends to a holomorphic function in a non-void $\mathbb{C}$-neighborhood of $u=0$, then it must be identically zero.

(b) If a function in $\operatorname{span}_{\mathbb{C}}\left\{\mathcal{F}_{2 k, b}(u), u \in(0, \varepsilon) \mid b \in \mathbb{Z} \cap(\{1\} \cup[k+2,2 k])\right\}$ (where $\left.0<\varepsilon<1\right)$ extends to a holomorphic function in a non-void $\mathbb{C}$-neighborhood of $u=0$, then it must be identically zero.

Proof. (a) From $\sup _{t>0} t^{\varepsilon} I_{0}(t) K_{0}(t)<\infty$ for any $\varepsilon \in(0,1]$, we can deduce that (cf. [49, (2.27), (2.30)]

$$
\begin{aligned}
& \int_{0}^{\infty} K_{0}(\sqrt{u} t)\left[I_{0}(t) K_{0}(t)\right]^{k} t^{2 \ell-1} \mathrm{~d} t=O\left(\int_{0}^{\infty} K_{0}(\sqrt{u} t) \mathrm{d} t\right)=O\left(\frac{1}{\sqrt{u}}\right), \\
& \int_{0}^{\infty} \sqrt{u} K_{1}(\sqrt{u} t)\left[I_{0}(t) K_{0}(t)\right]^{k} t^{2 \ell} \mathrm{d} t=O\left(\int_{0}^{\infty} \sqrt{u} K_{1}(\sqrt{u} t) t \mathrm{~d} t\right)=O\left(\frac{1}{\sqrt{u}}\right),
\end{aligned}
$$

when $2 \ell-1 \leq k$. Meanwhile, from Heaviside's integral formula [46, $\S 13.21(8)]$ for $\int_{0}^{\infty} K_{0}(t) t^{n-1} \mathrm{~d} t$, we have

$$
\begin{aligned}
& \frac{1}{2^{k}} \int_{0}^{\infty} K_{0}(\sqrt{u} t) t^{2 \ell-1-k} \mathrm{~d} t+\int_{0}^{\infty} K_{0}(\sqrt{u} t)\left\{\left[I_{0}(t) K_{0}(t)\right]^{k}-\frac{1}{(2 t)^{k}}\right\} t^{2 \ell-1} \mathrm{~d} t \\
= & \frac{\left[\Gamma\left(\ell-\frac{k}{2}\right)\right]^{2}}{4^{k-\ell+1}(\sqrt{u})^{2 \ell-k}}+O\left(\int_{0}^{\infty} K_{0}(\sqrt{u} t) t^{2 \ell-3-k} \mathrm{~d} t\right)
\end{aligned}
$$

when $2 \ell-1>k$, so the first half of (2.50) follows from induction on $\ell$. The second half of (2.50) founds on a similar argument. 
Recall the notation $h_{k, N}(z)$ for $k, N \in \mathbb{Z}_{>0}$ from (2.46) and set $h_{k, 0}(z)=\left[\frac{\pi}{2} H_{0}^{(1)}(z) H_{0}^{(2)}(z)\right]^{k}$. The following contour integral

$$
\begin{aligned}
& i(-1)^{\ell-1}\left(\frac{2}{\pi}\right)^{k+1} \int_{0}^{\infty} K_{0}(\sqrt{u} t)\left[K_{0}(t)\right]^{k}\left[K_{0}(t)-i \pi I_{0}(t)\right]^{k} t^{2 \ell-1} \mathrm{~d} t \\
& -\sum_{n \in \mathbb{Z} \cap\left[1, \max \left\{0,\left\lfloor\ell-\frac{k-1}{2}\right\rfloor\right\}\right]} \frac{(2 i)^{2(\ell-n)+1-k}\left[\Gamma\left(\ell-n+1-\frac{k}{2}\right)\right]^{2}}{\pi(\sqrt{u})^{2(\ell-n+1)-k}} \mathrm{H}_{k, n} \\
= & \int_{0}^{i \infty} H_{0}^{(1)}(\sqrt{u} z) h_{k, \max \left\{0,\left\lfloor\ell-\frac{k-1}{2}\right\rfloor\right\}}(z) z^{2 \ell-1} \mathrm{~d} z
\end{aligned}
$$

can be identified with

$$
\int_{0}^{\infty} H_{0}^{(1)}(\sqrt{u} x) h_{k, \max \left\{0,\left\lfloor\ell-\frac{k-1}{2}\right\rfloor\right\}}(x) x^{2 \ell-1} \mathrm{~d} x,
$$

because Jordan's lemma allows us to rotate the contour of integration $90^{\circ}$ clockwise, from the positive $\operatorname{Im} z$-axis to the positive $\operatorname{Re} z$-axis, as in [51, §2].

When $k$ is odd, we can relate the real parts of the last two displayed equations as follows:

$$
\begin{aligned}
& 2^{k}(-1)^{\ell-1}\left[(2 k+1) \mathcal{F}_{2 k-1,1}^{\ell}(u)-\frac{\widetilde{\mathbf{I}} \mathbf{M}(1,2 k, 2 \ell-1 \mid u)}{\pi^{k}}+\sum_{b=k+1}^{2 k-1} \frac{1+(-1)^{b-k}}{\left.(-1)^{\frac{b-k}{2}}\right]}\left(\begin{array}{c}
k \\
b-k+1
\end{array}\right) \mathcal{F}_{2 k-1, b}^{\ell}(u)\right] \\
& -(-1)^{\frac{k-1}{2}} 4^{\ell}\left(u D^{2}+D^{1}\right)^{\ell} \sum_{n \in \mathbb{Z} \cap\left[1, \max \left\{0, \ell-\frac{k-1}{2}\right\}\right]} \frac{\pi u^{n+\frac{k}{2}-1}(-1)^{n+1}}{2^{2 n+k-1}\left[\Gamma\left(n+\frac{k}{2}\right)\right]^{2}} \mathrm{H}_{k, n} \\
= & \int_{0}^{\infty} J_{0}(\sqrt{u} x) h_{k, \max \left\{0, \ell-\frac{k-1}{2}\right\}}(x) x^{2 \ell-1} \mathrm{~d} x,
\end{aligned}
$$

where $J_{0}(z)=\frac{1}{2}\left[H_{0}^{(1)}(z)+H_{0}^{(2)}(z)\right]$ is the Bessel function of the first kind, satisfying $\left|J_{0}(x)\right| \leq 1$ for all real-valued $x$. Here, the function $h_{k, \max \left\{0, \ell-\frac{k-1}{2}\right\}}(x) x^{2 \ell-1}$ is bounded in the $x \rightarrow 0^{+}$regime, and has $O\left(x^{-2}\right)$ behavior as $x \rightarrow \infty$, so we can invoke the dominated convergence theorem to show that

$$
\lim _{u \rightarrow 0^{+}} \int_{0}^{\infty} J_{0}(\sqrt{u} x) h_{k, \max \left\{0, \ell-\frac{k-1}{2}\right\}}(x) x^{2 \ell-1} \mathrm{~d} x=\int_{0}^{\infty} h_{k, \max \left\{0, \ell-\frac{k-1}{2}\right\}}(x) x^{2 \ell-1} \mathrm{~d} x .
$$

After rotating the contour of integration $90^{\circ}$ counterclockwise, we can identify the right-hand side of the last equation with

$$
\begin{aligned}
& \operatorname{Re} \int_{0}^{i \infty} h_{k, \max \left\{0, \ell-\frac{k-1}{2}\right\}}(z) z^{2 \ell-1} \mathrm{~d} z \\
= & (-1)^{\ell}\left(\frac{2}{\pi}\right)^{k} \operatorname{Re} \int_{0}^{\infty}\left[K_{0}(t)\right]^{k}\left[K_{0}(t)-i \pi I_{0}(t)\right]^{k} t^{2 \ell-1} \mathrm{~d} t \\
= & 2^{k}(-1)^{\ell} \lim _{u \rightarrow 0^{+}}\left[\frac{\widetilde{\mathbf{I} K M}(1,2 k, 2 \ell-1 \mid u)}{\pi^{k}}+\sum_{b=2}^{k} \frac{1+(-1)^{b-1}}{2(-1)^{\left\lfloor\frac{b-1}{2}\right\rfloor}}\left(\begin{array}{c}
k \\
b-1
\end{array}\right) \mathcal{F}_{2 k-1, b}^{\ell}(u)\right] .
\end{aligned}
$$

So far, we have already arrived at a precursor to (2.51), namely $\lim _{u \rightarrow 0^{+}}\left[O_{k}^{\ell}(u)-O_{k}^{\ell}(u)\right]=0$, where $O_{\sim}^{\ell}(u)$ denotes the right-hand side of (2.51). This precursor can be rewritten as

$$
\lim _{u \rightarrow 0^{+}}\left(u D^{2}+D^{1}\right)^{\ell-1}\left[O_{k}^{1}(u)-O_{k}^{1}(u)\right]=0,
$$

for all $\ell \in \mathbb{Z}_{>0}$, since we have $O_{k}^{\ell}(u)=4^{\ell-1}\left(u D^{2}+D^{1}\right)^{\ell-1} O_{k}^{1}(u)$ by the Bessel differential equation. Now that $O_{k}^{1}(u), u \in\left(0, \varepsilon_{k}\right)$ is equal to a generalized power series (by Corollary 2.2) for a suitably chosen $\varepsilon_{k} \in(0,1)$, and so is $O_{k}^{1}(u), u \in(0,1)$ (by its construction and the dominated convergence 
theorem), the vanishing derivatives in (2.61) bring us $O_{k}^{1}(u)=O_{\sim}^{1}(u), u \in\left(0, \varepsilon_{k}\right)$. By real-analytic continuations and differentiations, we can establish (2.51) and (2.51) in their entirety.

When $k$ is even, we can identify the imaginary parts of (2.56) and (2.57) as follows:

$$
\begin{aligned}
& 2^{k}(-1)^{\ell-1}\left[\frac{2 \mathbf{I} \widetilde{\mathbf{K}} \mathbf{M}(0,2 k+1,2 \ell-1 \mid u)}{\pi^{k+1}}-\sum_{b=k+1}^{2 k-1} \frac{1-(-1)^{b-k}}{(-1)^{\left\lfloor\frac{b-k}{2}\right\rfloor}}\left(\begin{array}{c}
k \\
b-k+1
\end{array}\right) \mathcal{F}_{2 k-1, b}^{\ell}(u)\right] \\
& -\sum_{n \in \mathbb{Z} \cap\left[1, \max \left\{0, \ell-\frac{k}{2}\right\}\right]} \frac{(-4)^{\ell-n} 2^{1-k}\left[\left(\ell-n-\frac{k}{2}\right) !\right]^{2}}{(-1)^{k / 2} \pi u^{\ell-n+1-\frac{k}{2}}} \mathrm{H}_{k, n} \\
= & \int_{0}^{\infty} Y_{0}(\sqrt{u} x) h_{k, \max \left\{0, \ell-\frac{k}{2}\right\}}(x) x^{2 \ell-1} \mathrm{~d} x,
\end{aligned}
$$

where $Y_{0}(z)=\frac{1}{2 i}\left[H_{0}^{(1)}(z)-H_{0}^{(2)}(z)\right]$ is the Bessel function of the second kind. Meanwhile, the real parts of (2.56) and (2.57) leave us

$$
\begin{aligned}
& 2^{k}(-1)^{\ell-1}\left[(2 k+1) \mathcal{F}_{2 k-1,1}^{\ell}(u)-\frac{\widetilde{\mathbf{I K M}}(1,2 k, 2 \ell-1 \mid u)}{\pi^{k}}+\sum_{b=k+1}^{2 k-1} \frac{1+(-1)^{b-k}}{(-1)^{\left\lfloor\frac{b-k}{2}\right\rfloor}}\left(\begin{array}{c}
k \\
b-k+1
\end{array}\right) \mathcal{F}_{2 k-1, b}^{\ell}(u)\right] \\
= & \int_{0}^{\infty} J_{0}(\sqrt{u} x) h_{k, \max \left\{0, \ell-\frac{k}{2}\right\}}(x) x^{2 \ell-1} \mathrm{~d} x,
\end{aligned}
$$

which is subtly different from (2.58). Here, we point out that

$$
\begin{aligned}
& \mathbf{\mathbf { K }} \mathbf{M}(0,2 k+1,2 \ell-1 \mid u)+\widetilde{\mathbf{I}} \mathbf{K M}(1,2 k, 2 \ell-1 \mid u) \log \frac{\sqrt{u}}{2} \\
= & \int_{0}^{\infty}\left[K_{0}(\sqrt{u} t)+I_{0}(\sqrt{u} t) \log \frac{\sqrt{u}}{2}\right]\left[K_{0}(t)\right]^{2 k} t^{2 \ell-1} \mathrm{~d} t
\end{aligned}
$$

equals its own Maclaurin series for $u \in\left(0,(2 k)^{2}\right)$, because the dominated convergence theorem allows us to perform termwise integration on the convergent series [46, §3.71(14)]

$$
K_{0}(\sqrt{u} t)+I_{0}(\sqrt{u} t) \log \frac{\sqrt{u} t}{2}=\sum_{m=0}^{\infty} \frac{\psi^{(0)}(m+1)}{(m !)^{2}}\left(\frac{u t^{2}}{4}\right)^{m}
$$

where $\psi^{(0)}(m+1):=\frac{1}{m !} \int_{0}^{\infty} x^{m} e^{-x} \log x \mathrm{~d} x$ goes like $\log m+O\left(\frac{1}{m}\right)$ as $m \rightarrow \infty$. As we may recall from [46, §3.51], the following limit

$$
\lim _{u \rightarrow 0^{+}}\left[Y_{0}(\sqrt{u} x)-\frac{2}{\pi} J_{0}(\sqrt{u} x) \log \frac{\sqrt{u}}{2}\right]=\frac{2\left(\gamma_{0}+\log x\right)}{\pi}
$$

holds for all $x>0$, where $\gamma_{0}=-\psi^{(0)}(1)$ is the Euler-Mascheroni constant. Meanwhile, we note that the function $\widetilde{h}_{k, \ell}(x) x^{2 \ell-1}:=h_{k, \max \left\{0, \ell-\frac{k}{2}\right\}}(x) x^{2 \ell-1}-\mathrm{H}_{k, \max \left\{0, \ell+1-\frac{k}{2}\right\}} h_{2,0}(x) x$ is bounded in the $x \rightarrow 0^{+}$ regime, and has $O\left(x^{-3}\right)$ behavior as $x \rightarrow \infty$, so the dominated convergence theorem allows us to conclude that

$$
\lim _{u \rightarrow 0^{+}} \int_{0}^{\infty}\left[Y_{0}(\sqrt{u} x)-\frac{2}{\pi} J_{0}(\sqrt{u} x) \log \frac{\sqrt{u}}{2}\right] \widetilde{h}_{k, \ell}(x) x^{2 \ell-1} \mathrm{~d} x=\frac{2}{\pi} \int_{0}^{\infty} \widetilde{h}_{k, \ell}(x) z^{2 \ell-1}\left(\gamma_{0}+\log x\right) \mathrm{d} x
$$

is a finite real number. To compensate for the difference $h_{k, \max \left\{0, \ell-\frac{k}{2}\right\}}(x) x^{2 \ell-1}-\widetilde{h}_{k, \ell}(x) x^{2 \ell-1}$, we need the explicit knowledge that (cf. [52, Lemma 3.2])

$$
\mathcal{F}_{3,1}(u)=\frac{\sqrt{3}}{20 \pi^{1 / 2}} \frac{1}{2 \pi i} \int_{\frac{1}{4}-i \infty}^{\frac{1}{4}+i \infty} \frac{\Gamma\left(\frac{1}{3}-s\right) \Gamma\left(\frac{2}{3}-s\right)[\Gamma(s)]^{2}}{(4-u) \Gamma(1-s) \Gamma\left(s+\frac{1}{2}\right)}\left[\frac{108 u}{(4-u)^{3}}\right]^{-s} \mathrm{~d} s
$$




$$
\begin{aligned}
& =-\frac{\log \frac{u}{(4-u)^{3}}}{10(4-u)}+\frac{O\left(\frac{u}{(4-u)^{3}}\right)}{4-u}, \\
\mathcal{F}_{3,3}(u) & =\frac{\sqrt{3}}{8 \pi^{5 / 2}} \frac{1}{2 \pi i} \int_{\frac{1}{4}-i \infty}^{\frac{1}{4}+i \infty} \frac{\Gamma\left(\frac{1}{3}-s\right) \Gamma\left(\frac{1}{2}-s\right) \Gamma\left(\frac{2}{3}-s\right)[\Gamma(s)]^{3}}{4-u}\left[\frac{108 u}{(4-u)^{3}}\right]^{-s} \mathrm{~d} s-\mathcal{F}_{3,2}(u) \\
& =\frac{\pi^{2}+3 \log ^{2} \frac{u}{(4-u)^{3}}}{24 \pi(4-u)}+\frac{O\left(\frac{u}{(4-u)^{3}}\right)}{4-u}
\end{aligned}
$$

hold in the $u \rightarrow 0^{+}$regime (where the logarithmic terms are attributed to residues at $s=0$ ), so as to deduce

$$
\begin{aligned}
& \mathrm{H}_{k, \max \left\{0, \ell+1-\frac{k}{2}\right\}} \int_{0}^{\infty}\left[Y_{0}(\sqrt{u} x)-\frac{2}{\pi} J_{0}(\sqrt{u} x) \log \frac{\sqrt{u}}{2}\right] h_{2,0}(x) x \mathrm{~d} x \\
= & \mathrm{H}_{k, \max \left\{0, \ell+1-\frac{k}{2}\right\}}\left\{-8 \mathcal{F}_{3,3}(u)-\frac{40}{\pi} \mathcal{F}_{3,1}(u) \log \frac{\sqrt{u}}{2}+\right. \\
& \left.+\left(\frac{2}{\pi}\right)^{3} \int_{0}^{\infty}\left[K_{0}(\sqrt{u} t)+I_{0}(\sqrt{u} t) \log \frac{\sqrt{u}}{2}\right]\left[K_{0}(t)\right]^{4} t \mathrm{~d} t\right\} \\
= & \frac{2^{k}(-1)^{\ell} \log ^{2} \frac{\sqrt{u}}{2}}{\pi^{2}} \sum_{b=2}^{k} \frac{1+(-1)^{b}}{(-1)^{\left\lfloor\frac{b}{2}\right\rfloor}}\left(\begin{array}{c}
k \\
b-1
\end{array}\right) \mathcal{F}_{2 k-1, b}^{\ell}(0)+O(1) .
\end{aligned}
$$

Here, in the last step, we have combined (2.47) with the generalized Bailey-Borwein-BroadhurstGlasser sum rule [53, (5)] into the following form for $k \in 2 \mathbb{Z}_{>0}$ and $\ell \in \mathbb{Z}_{>0}$ :

$$
(-1)^{\ell-\frac{k}{2}} \mathrm{H}_{k, \max \left\{0, \ell+1-\frac{k}{2}\right\}}=\int_{0}^{\infty} \frac{\left[\pi I_{0}(t)+i K_{0}(t)\right]^{k}-\left[\pi I_{0}(t)-i K_{0}(t)\right]^{k}}{\pi i}\left[\frac{2 K_{0}(t)}{\pi}\right]^{k} t^{2 \ell-1} \mathrm{~d} t .
$$

Let $E_{k}^{\ell}(u)$ be the finite sum on the right-hand side of (2.52), then the efforts so far lead us to $E_{k}^{\ell}(u)-$ $\underset{\sim}{E}(u)=O(1)$ as $u \rightarrow 0^{+}$. Before proceeding further, we note that the sequence

$$
\mathrm{b}_{n}(u, t):=\left[t^{2 n}-4^{n}\left(u D^{2}+D^{1}\right)^{n}\right]\left[K_{0}(\sqrt{u} t) \log \frac{\sqrt{u}}{2}+\frac{1}{2} I_{0}(\sqrt{u} t) \log ^{2} \frac{\sqrt{u}}{2}\right]
$$

satisfies a recursion

$$
4\left(u D^{2}+D^{1}\right) \mathrm{b}_{n}(u, t)-\mathrm{b}_{n+1}(u, t)=\frac{t^{2 n}}{u}\left\{I_{0}(\sqrt{u} t)+2 \sqrt{u} t\left[I_{1}(\sqrt{u} t) \log \frac{\sqrt{u}}{2}-K_{1}(\sqrt{u} t)\right]\right\}
$$

with the initial condition $\mathrm{b}_{0}(u, t)=0$, and [46, §3.71(15)]

$$
\sqrt{u} t\left[I_{1}(\sqrt{u} t) \log \frac{\sqrt{u} t}{2}-K_{1}(\sqrt{u} t)\right]=-1+\frac{1}{2} \sum_{m=0}^{\infty} \frac{\psi^{(0)}(m+1)+\psi^{(0)}(m+2)}{m !(m+1) !}\left(\frac{u t^{2}}{4}\right)^{m+1},
$$

so we can show inductively that

$$
\mathrm{b}_{n}(u, t)-\sum_{m=1}^{n} \frac{4^{m-1}[(m-1) !]^{2}}{u^{m}} t^{2(n-m)}=\sum_{m=0}^{\infty} \mathrm{a}_{n, m}(t) u^{m}
$$

defines a holomorphic function in $u \in \mathbb{C}$ for each fixed $t \in(0, \infty)$ and $n \in \mathbb{Z}_{\geq 0}$. Therefore, we have $E_{k}^{\ell}(u)-E_{\sim}^{\ell}(u)=4^{\ell-1}\left(u D^{2}+D^{1}\right)^{\ell-1} E_{k}^{1}(u)+O(1)$ as $u \rightarrow 0^{+}$. By Corollary 2.2, there exists a suitable $\varepsilon_{k} \in(0,1)$ such that the function $E_{k}^{1}(u), u \in\left(0, \varepsilon_{k}\right)$ equals a generalized power series in (fractional) powers of $u$ and $\log u$; such a series is in fact a Maclaurin series, the coefficients of which are computable from the finite numbers $\lim _{u \rightarrow 0^{+}}\left(u D^{2}+D^{1}\right)^{\ell-1} E_{k}^{1}(u)$ for $\ell \in \mathbb{Z}_{>0}$. This reveals $E_{k}^{1}(u)=\mathscr{E}_{k}^{1}(u)$ as a holomorphic function in a non-void $\mathbb{C}$-neighborhood of $u=0$. The full version of (2.52) then 
follows from repeated applications of the Bessel differentiation operator $u D^{2}+D^{1}$ and invocations of the Laurent expansion for $\mathrm{b}_{n}(u, t)$, as given in (2.75). To prove (2.52), we differentiate (2.52) while noting that

$$
\begin{aligned}
& 2 u D^{1}\left[K_{0}(\sqrt{u} t) \log \frac{\sqrt{u}}{2}+\frac{1}{2} I_{0}(\sqrt{u} t) \log ^{2} \frac{\sqrt{u}}{2}\right]-\sqrt{u} t\left[-K_{1}(\sqrt{u} t) \log \frac{\sqrt{u}}{2}+\frac{1}{2} I_{1}(\sqrt{u} t) \log ^{2} \frac{\sqrt{u}}{2}\right] \\
= & K_{0}(\sqrt{u} t)+I_{0}(\sqrt{u} t) \log \frac{\sqrt{u}}{2}
\end{aligned}
$$

extends to a holomorphic function of $u \in \mathbb{C}$.

According to (2.65), we know that $\pi \mathcal{F}_{2 k-1,1}(u)+\frac{2 k}{2 k+1} \mathcal{F}_{2 k-1,2}(u) \log \frac{\sqrt{u}}{2}$ and $\pi \mathcal{F}_{2 k-1, b}(u)+$ $\mathcal{F}_{2 k-1, b-k+2}(u) \log \frac{\sqrt{u}}{2}$ for $b \in \mathbb{Z} \cap[k+1,2 k-2]$ are holomorphic in a non-void $\mathbb{C}$-neighborhood of $u=0$. From (2.51) and (2.52), we know that the generalized power series expansion for $\mathcal{F}_{2 k-1,2 k-1}(u)$ additionally involve $u^{\mathbb{Z}+\frac{1}{2}}$ or $u^{\mathbb{Z}} \log ^{2} \frac{\sqrt{u}}{2}$ terms, according as $k$ is odd or even.

If a linear combination of these functions [say, $\left.c_{1} \pi^{k} \mathcal{F}_{2 k-1,1}(u)+\sum_{b=k+1}^{2 k-1} c_{b} \pi^{2 k-b} \mathcal{F}_{2 k-1, b}(u)\right]$ is holomorphic near the origin, then it should not involve $u^{\mathbb{Z}+\frac{1}{2}}$ or $u^{\mathbb{Z}} \log ^{2} \frac{\sqrt{u}}{2}$ terms, hence $c_{2 k-1}=0$. Furthermore, we require that in the generalized power series expansion for $c_{1} \pi^{k} \mathcal{F}_{2 k-1,1}(u)+\sum_{b=k+1}^{2 k-2} c_{b} \pi^{2 k-b}$ $\mathcal{F}_{2 k-1, b}(u)$, the coefficients for $u^{n} \log \frac{\sqrt{u}}{2}, n \in \mathbb{Z} \cap[0, k-2]$ should all vanish, that is,

$$
\frac{2 k c_{1} \pi^{k-1}}{2 k+1} \mathcal{F}_{2 k-2,1}(1)+\sum_{b=k+1}^{2 k-2} \frac{c_{b} \mathcal{F}_{2 k-2, b-k+1}(1)}{\pi^{b-2 k+1}}=0, \quad \ell \in \mathbb{Z} \cap[1, k-1]
$$

Since $\operatorname{det} \mathbf{N}_{k-1}=\operatorname{det}\left(\pi^{k+\frac{1}{2}-b} \mathcal{F}_{2 k-2, b}^{\ell}(1)\right)_{1 \leq b, \ell \leq k-1} \neq 0$, we must have $c_{b}=0$ for $b \in \mathbb{Z} \cap(\{1\} \cup[k+1,2 k-$ 2]).

(b) In a non-void $\mathbb{C}$-neighborhood of $u=0$, we have holomorphic functions $\pi \mathcal{F}_{2 k, 1}(u)+\frac{2 k+1}{2 k+2} \mathcal{F}_{2 k, 2}(u) \log \frac{\sqrt{u}}{2}$ and $\pi \mathcal{F}_{2 k, b}(u)+\mathcal{F}_{2 k, b-k+1}(u) \log \frac{\sqrt{u}}{2}$ for $b \in \mathbb{Z} \cap[k+2,2 k]$. The conclusion follows from the coefficients for $u^{n} \log \frac{\sqrt{u}}{2}, n \in \mathbb{Z} \cap[0, k-1]$ and the fact that $\operatorname{det} \mathbf{M}_{k}=\operatorname{det}\left(\pi^{k+1-b} \mathcal{F}_{2 k-1, b}^{\ell}(1)\right)_{1 \leq b, \ell \leq k} \neq 0$.

Corollary 2.11 (Determination of $\mathbf{S}_{3}$ ). The matrix

$$
\mathbf{S}_{3}=\left(\begin{array}{rrr}
-25 & & \\
& -\frac{4}{3} & 4 \\
4 &
\end{array}\right)
$$

fits into a special case of (2.36), namely

$$
\frac{\sqrt{\left|\mathcal{L}_{3}(u)\right|}}{\Lambda_{3}}\left(\begin{array}{r}
W\left[\mathcal{F}_{3,2}(u), \mathcal{F}_{3,3}(u)\right] \\
-W\left[\mathcal{F}_{3,1}(u), \mathcal{F}_{3,3}(u)\right] \\
W\left[\mathcal{F}_{3,1}(u), \mathcal{F}_{3,2}(u)\right]
\end{array}\right)=\mathbf{S}_{3}\left(\begin{array}{c}
\mathcal{F}_{3,1}(u) \\
\mathcal{F}_{3,2}(u) \\
\mathcal{F}_{3,3}(u)
\end{array}\right)
$$

for $\left|\mathcal{L}_{3}(u)\right|=u^{2}(4-u)(16-u), u \in(0,4)$ and $\Lambda_{3}=\frac{1}{20}$.

Proof. During the proof of [49, Proposition 5.5], we have already pointed out that the left-hand side of (2.79) is annihilated by $\widetilde{L}_{3}$ (which is a special case of Proposition 3.1 below), and have evaluated the first row of $\mathbf{S}_{3}$. 
To compute the last row of $\mathbf{S}_{3}$, simply check that $\widetilde{\mathbf{I K M}}(2,3 ; 1 \mid u)=\mathbf{I K M}(1,3 ; 1)\left[1+O\left(u^{2}\right)\right]=\frac{\pi^{2}}{16}[1+$ $\left.O\left(u^{2}\right)\right][3,(55)]$ as well as

$$
\begin{aligned}
& \lim _{u \rightarrow 0^{+}} \sqrt{\left|\mathcal{L}_{3}(u)\right|} W\left[\mathcal{F}_{3,1}(u), \mathcal{F}_{3,2}(u)=\lim _{u \rightarrow 0^{+}} \frac{32}{5 \pi^{3}} \operatorname{det}\left(\begin{array}{rr}
D^{0} \widetilde{\mathbf{I}} \mathbf{M}(1,4 ; 1 \mid u) & D^{0} \widetilde{\mathbf{I}} \mathbf{K M}(2,3 ; 1 \mid u) \\
u D^{1} \widetilde{\mathbf{I}} \mathbf{M}(1,4 ; 1 \mid u) & u D^{1} \widetilde{\mathbf{I}} \mathbf{K}(2,3 ; 1 \mid u)
\end{array}\right)\right. \\
= & \lim _{u \rightarrow 0^{+}} \frac{32}{5 \pi^{3}} \operatorname{det}\left(\begin{array}{rr}
-\frac{\pi^{2} \log u}{32}+O(u \log u) & \frac{\pi^{2}}{16}+O\left(u^{2}\right) \\
-\frac{\pi^{2}}{32}+O(u \log u) & O(u)
\end{array}\right)=\frac{\pi}{80}=\frac{1}{5} \mathcal{F}_{3,2}(0) .
\end{aligned}
$$

This accounts for the only non-vanishing element in the last row of $\mathbf{S}_{3}$, since any non-trivial linear combination of $\mathcal{F}_{3,1}(u)=-\frac{\log u}{40}[1+O(u)]$ (cf. [3, (55)] or (2.68) above) and $\mathcal{F}_{3,3}(u)=\frac{\log ^{2} u}{32 \pi}+O(\log u)$ (cf. [49, (5.43)] or (2.69) above) in the $u \rightarrow 0^{+}$regime is incompatible with such a finite limit. [To argue for $\mathbf{S}_{2 k-1}, k \in \mathbb{Z}_{>2}$, one will need sum rules (2.51) and (2.52) in Proposition 2.10, instead of the explicit formulae like (2.68) and (2.69).]

To compute the second row of $\mathbf{S}_{3}$, we quote in advance the symmetry $\mathbf{S}_{3}=\mathbf{S}_{3}^{\mathrm{T}}$ from Corollary 3.3 , which enables us to set up an equation $-\sqrt{\left|\mathcal{L}_{3}(u)\right|} W\left[\mathcal{F}_{3,1}(u), \mathcal{F}_{3,3}(u)\right]=c \mathcal{F}_{3,2}(u)+\frac{1}{5} \mathcal{F}_{3,3}(u)$ for a certain constant $c$. We observe that $\lim _{u \rightarrow 4^{-}} \sqrt{\left|\mathcal{L}_{3}(u)\right|} W\left[\mathcal{F}_{3,1}(u), \mathcal{F}_{3,3}(u)\right]=0$ (since $W\left[\mathcal{F}_{3,1}(u), \mathcal{F}_{3,3}(u)\right]$ is holomorphic in a neighborhood of $u=4$ ) and that $\mathcal{F}_{3,2}(4)-3 \mathcal{F}_{3,3}(4)=0$ follows from the sum rule $\operatorname{Im} F_{2,1}^{1}(4)=0$, a special case of Proposition 2.9(a). Thus, we must have $c=-\frac{1}{15}$.

\section{3. $\mathbf{W}^{\star}$ ALGEBRA}

The main purpose of this section is to construct analogs of Corollary 2.11 for matrices of all sizes, and explore their consequences. In other words, for each $m \in \mathbb{Z}_{>2}$, we will build a matrix $\mathbf{S}_{m} \in \mathbb{Q}^{m \times m}$ that fits into (2.36) - the rational entries of $\mathbf{S}_{m}$ enable us to express the Wrońskian determinant $W\left[f_{1}(u), \ldots, f_{m-1}(u)\right]$ as a $\mathbb{Q}$-linear combination of $\left\{\left|\mathcal{L}_{m}(u)\right|^{(2-m) / 2} \mathcal{F}_{m, j}(u) \mid j \in \mathbb{Z} \cap[1, m]\right\}$, whenever $\left\{f_{n} \mid n \in \mathbb{Z} \cap[1, m-1]\right\}$ is a subset of $\left\{\mathcal{F}_{m, j} \mid j \in \mathbb{Z} \cap[1, m]\right\}$.

This master plan decomposes into several subtasks to be completed in the subsections to follow.

In $\$ 3.1$, we study algebraically all the cofactors of the Wrońskian matrix $\mathbf{W}\left[h_{1}(u), \ldots, h_{m}(u)\right]$, where $h_{1}(u), \ldots, h_{m}(u)$ are annihilated by a differential operator $\widetilde{L}_{m}=\sum_{j=0}^{m} \ell_{m, j}(u) D^{j}=(-1)^{m} \widetilde{L}_{m}^{*}$, with smooth coefficients $\ell_{m, j}(u)$ The algebraic properties of these cofactors account for not only the Vanhove matrix $\mathbf{V}_{m}(u)$ in Theorem 1.1, but also the parity relation $\mathbf{S}_{m}=(-1)^{m+1} \mathbf{S}_{m}^{\mathrm{T}}$.

In $\$ 3.2$, we scrutinize the adjugate matrix for $\mathbf{W}\left[\mathcal{F}_{m, 1}(u), \ldots, \mathcal{F}_{m, m}(u)\right]$ analytically, by revisiting some themes in $\$ 2$, namely Bessel differential equations and sum rules for Bessel moments. In particular, we will show that the local expansions of certain Wrońskian cofactors and certain linear combinations of $\mathcal{F}_{m, 1}(u), \ldots, \mathcal{F}_{m, m}(u)$ match each other, when $u$ approaches a threshold value.

In $\$ 3.3$, we prove Theorem 1.1 in its entirety, by carefully handling real-analytic continuations of Wrońskian cofactors across threshold values. In addition to proving the representation of $\mathbf{S}_{m}$ given by Theorem 1.1, we will also explore the recursive structures of the combinatorial sums therein. Such combinatorial analysis will prepare us for the inversion of $\mathbf{S}_{m}$ later in $\$ 4.1$

Simply put, in $\$ 3.1$ we settle the qualitative viability of 2.36 through differential equations, and augment it to $\mathbf{W}_{m}^{\star}(u)=\frac{\Lambda_{m}}{\left|\mathcal{L}_{m}(u)\right|^{(m-2) / 2}} \mathbf{S}_{m} \mathbf{W}_{m}^{\mathrm{T}}(u) \mathbf{V}_{m}(u)$; in $\$ 3.2$ we perform quantitative analysis on both sides of (2.36) near thresholds, preparing us for a complete calculation of $\mathbf{S}_{m}$ in $\$ 3.3$, as well as an off-shell quadratic relation that descends from $\mathbf{W}_{m}^{\star}(u)=\frac{\Lambda_{m}}{\left|\mathcal{L}_{m}(u)\right|^{m / 2}}\left[\mathbf{W}_{m}(u)\right]^{-1}$.

\footnotetext{
${ }^{9}$ Being indifferent to detailed structures of the polynomials $\ell_{m, j}(u)$, these algebraic mechanisms extend naturally to differential systems other than Vanhove's operators. It is our hope that such extensions will enable us to construct other types of quadratic relations among periods of connections [24], going beyond the cases of Bessel moments treated in [25] and the current work.
} 
3.1. Cofactors of Wrońskians. The adjugate $\mathbf{W}^{\star}$ of a Wrońskian matrix $\mathbf{W}=\mathbf{W}\left[f_{1}(u), \ldots, f_{m}(u)\right]$ is the transpose of its cofactor matrix cof $\mathbf{W}$. Each entry in the last column of $\mathbf{W}^{\star}=(\operatorname{cof} \mathbf{W})^{\mathrm{T}}$ is expressible as a Wrońskian determinant $W\left[f_{i_{1}}(u), \ldots, f_{i_{m-1}}(u)\right]$, where $\left\{i_{1}, \ldots, i_{m-1}\right\} \subset \mathbb{Z} \cap[1, m]$.

Proposition 3.1 (Vanhove duality). For any smooth differential operator $\widetilde{L}_{m}=\mathcal{L}_{m}(u) D^{m}+\cdots($ omitting lower order terms) satisfying the parity relation $\widetilde{L}_{m}^{*}=(-1)^{m} \widetilde{L}_{m}$, and $\left\{f_{n}(u), u \in I \mid n \in \mathbb{Z} \cap[1, m-1]\right\} \subset$ $C^{\infty}(I) \cap \operatorname{ker} \widetilde{L}_{m}$ for an open interval $I$, we have

$$
\widetilde{L}_{m}\left(\left|\mathcal{L}_{m}(u)\right|^{(m-2) / 2} W\left[f_{1}(u), \ldots, f_{m-1}(u)\right]\right)=0
$$

on the same open interval.

In particular, this is true when $\widetilde{L}_{m}$ is the $m$-th order Vanhove operator (defined in Proposition 2.1) with parity $(-1)^{m}$ (proved in Proposition 2.3), and $\mathcal{L}_{m}(u)$ is the polynomial specified by (1.8).

Proof. Without loss of generality, we may assume that the functions $f_{1}, \ldots, f_{m-1}$ are linearly independent. We may further assume that there exists a function $f_{m}$, such that $\operatorname{span}_{\mathbb{C}}\left\{f_{j}(u), u \in I \mid j \in \mathbb{Z} \cap[1, m]\right\}=$ $C^{\infty}(I) \cap \operatorname{ker} \widetilde{L}_{m}$.

Using Ince's notation [31, §5.21, p. 120] for Wrońskian determinants

$$
\Delta_{0}=1 ; \quad \Delta_{r}=W\left[f_{1}(u), \ldots, f_{r}(u)\right] \text { where } r \in \mathbb{Z} \cap[1, m],
$$

we have

$$
(-1)^{m} \frac{\widetilde{L}_{m} f(u)}{\mathcal{L}_{m}(u)}=\frac{W\left[f(u), f_{1}(u), \ldots, f_{m}(u)\right]}{\Delta_{m}}=(-1)^{m} \frac{\Delta_{m}}{\Delta_{m-1}} \frac{\mathrm{d}}{\mathrm{d} u} \frac{\Delta_{m-1}^{2}}{\Delta_{m} \Delta_{m-2}} \frac{\mathrm{d}}{\mathrm{d} u} \cdots \frac{\mathrm{d}}{\mathrm{d} u} \frac{\Delta_{1}^{2}}{\Delta_{2} \Delta_{0}} \frac{\mathrm{d}}{\mathrm{d} u} \frac{\Delta_{0} f(u)}{\Delta_{1}} .
$$

From the combinatorial constraint (2.13) that directly descends from the parity relation $\widetilde{L}_{m}^{*}=(-1)^{m} \widetilde{L}_{m}$, we conclude that $\widetilde{L}_{m}=\mathcal{L}_{m}(u) D^{m}+\frac{m}{2} \frac{\mathrm{d} \mathcal{L}_{m}(u)}{\mathrm{d} u} D^{m-1} \ldots$ [cf. (2.3)], up to sub-leading order, so $\Delta_{m}$ is proportional to $\left|\mathcal{L}_{m}(u)\right|^{-m / 2}$. In view of the linear independence of $f_{1}, \ldots, f_{m}$, we know that $\Delta_{m}=C\left|\mathcal{L}_{m}(u)\right|^{-m / 2}, u \in$ $I$ for a non-vanishing constant $C$. Appealing again to the parity relation $\widetilde{L}_{m}^{*}=(-1)^{m} \widetilde{L}_{m}$, we subsequently have

$$
\widetilde{L}_{m} f(u)=\frac{C \Delta_{0}}{\Delta_{1}} \frac{\mathrm{d}}{\mathrm{d} u} \frac{\Delta_{1}^{2}}{\Delta_{2} \Delta_{0}} \frac{\mathrm{d}}{\mathrm{d} u} \cdots \frac{\mathrm{d}}{\mathrm{d} u} \frac{\Delta_{m-1}^{2}}{\Delta_{m} \Delta_{m-2}} \frac{\mathrm{d}}{\mathrm{d} u} \frac{\mathcal{L}_{m}(u) f(u)}{\Delta_{m-1}\left|\mathcal{L}_{m}(u)\right|^{m / 2}},
$$

so (3.1) follows immediately.

Remark The Vanhove duality relations for Vanhove's operators $\widetilde{L}_{3}$ and $\widetilde{L}_{4}$ (see Table \) have already appeared in [49, (5.34) and (5.67)].

In the next proposition, we show that all the cofactors of $\mathbf{W}_{m}$ are expressible via linear combinations of fundamental solutions to Vanhove's differential equation, together with their derivatives.

Proposition 3.2 (Vanhove matrix $\mathbf{V}_{m}(u)$ ). Fix an integer $m \in \mathbb{Z}_{>1}$. For a smooth differential operator $\widetilde{L}_{m}=\sum_{j=0}^{m} \ell_{m, j}(u) D^{j}=(-1)^{m} \widetilde{L}_{m}^{*}$ where $\ell_{m, j} \in C^{\infty}(I)$, consider $f_{1}, \ldots, f_{m-1} \in C^{\infty}(I) \cap \operatorname{ker} \widetilde{L}_{m}$. Define

$$
F_{r}(u):=\left|\ell_{m, m}(u)\right|^{\frac{m-2}{2}} \operatorname{det}\left(D^{i-1} f_{j}(u)\right)_{i \in \mathbb{Z} \cap([1, m-1-r] \cup[m+1-r, m]), j \in \mathbb{Z} \cap[1, m-1]}
$$

for $r \in \mathbb{Z} \cap[0, m-1]$, where all the row indices are arranged in increasing order (even when they are not consecutive integers). We have

$$
\begin{aligned}
& \left((-1)^{m-1} F_{m-1}(u), \ldots,(-1)^{r} F_{r}(u), \ldots, F_{0}(u)\right) \\
= & (-1)^{m+1}\left(D^{0} F_{0}(u), \ldots, D^{m-r} F_{0}(u), \ldots, D^{m-1} F_{0}(u)\right) \mathbf{V}_{m}^{\mathrm{T}}(u)
\end{aligned}
$$

where

$$
\left(\mathbf{V}_{m}(u)\right)_{a, b}:=\sum_{n=a+b-1}^{m}(-1)^{a+n+m+1}\left(\begin{array}{l}
n-a \\
b-1
\end{array}\right) \frac{D^{n-a-b+1} \ell_{m, n}(u)}{\ell_{m, m}(u)}
$$

for $a, b \in \mathbb{Z} \cap[1, m]$. 
In particular, when $\widetilde{L}_{m}$ is the m-th order Vanhove operator (defined in Proposition 2.1) with parity $(-1)^{m}$ (proved in Proposition 2.3), this explains the origin of the Vanhove matrix $\mathbf{V}_{m}(u)$ defined in (1.11). Proof. Set $\mathcal{L}_{m}(u):=\ell_{m, m}(u)$. If $f_{1}, \ldots, f_{m-1} \in C^{\infty}(I) \cap \operatorname{ker} \widetilde{L}_{m}$, then the first-order derivative of

$$
F_{0}(u):=\left|\mathcal{L}_{m}(u)\right|^{\frac{m-2}{2}} \operatorname{det}\left(D^{i-1} f_{j}(u)\right)_{i, j \in \mathbb{Z} \cap[1, m-1]}=\left|\mathcal{L}_{m}(u)\right|^{\frac{m-2}{2}} W\left[f_{1}(u), \ldots, f_{m-1}(u)\right] \in C^{\infty}(I) \cap \operatorname{ker} \widetilde{L}_{m}
$$

reads

$$
\begin{aligned}
D^{1} F_{0}(u) & =\left|\mathcal{L}_{m}(u)\right|^{\frac{m-2}{2}} \operatorname{det}\left(D^{i-1} f_{j}(u)\right)_{i \in \mathbb{Z} \cap([1, m-2] \cup\{m\}), j \in \mathbb{Z} \cap[1, m-1]}+\frac{m-2}{2} \frac{F_{0}(u)}{\mathcal{L}_{m}(u)} D^{1} \mathcal{L}_{m}(u) \\
& =F_{1}(u)+\frac{m-2}{2} \frac{F_{0}(u)}{\mathcal{L}_{m}(u)} D^{1} \mathcal{L}_{m}(u) .
\end{aligned}
$$

For higher order derivatives, we have the following recursion:

$$
\begin{aligned}
\frac{F_{r}(u)}{\left|\mathcal{L}_{m}(u)\right|^{\frac{m-2}{2}}:=} & \operatorname{det}\left(D^{i-1} f_{j}(u)\right)_{i \in \mathbb{Z} \cap([1, m-1-r] \cup[m+1-r, m]), j \in \mathbb{Z} \cap[1, m-1]} \\
= & D^{1} \operatorname{det}\left(D^{i-1} f_{j}(u)\right)_{i \in \mathbb{Z} \cap([1, m-r] \cup[m+2-r, m]), j \in \mathbb{Z} \cap[1, m-1]} \\
& -\operatorname{det}\left(D^{i-1} f_{j}(u)\right)_{i \in \mathbb{Z} \cap([1, m-r] \cup[m+2-r, m-1] \cup\{m+1\}), j \in \mathbb{Z} \cap[1, m-1]}
\end{aligned}
$$

according to fundamental properties of determinants. Upon invoking the differential equations $\widetilde{L}_{m} f_{j}(u)=$ $0, j \in \mathbb{Z} \cap[1, m-1]$, we can further convert this recursion into

$$
F_{r}(u)=D^{1} F_{r-1}(u)+\frac{F_{r-1}(u)}{\mathcal{L}_{m}(u)} D^{1} \mathcal{L}_{m}(u)+\frac{(-1)^{r} \ell_{m, m-r}(u)}{\mathcal{L}_{m}(u)} F_{0}(u) .
$$

Such a recursion is actually retroactively compatible with the $r=1$ case in (3.9), since $\ell_{m, m-1}(u)=$ $\frac{m}{2} \frac{\mathrm{d}}{\mathrm{d} u} \mathcal{L}_{m}(u)$ is a consequence of the parity relation $\widetilde{L}_{m}=\sum_{j=0}^{m} \ell_{m, j}(u) D^{j}=(-1)^{m} \widetilde{L}_{m}^{*}$. In other words, to build a linear combination

$$
F_{r}(u)=\sum_{q} \varphi_{r, q}(u) D^{q} F_{0}(u)
$$

for $r \in \mathbb{Z} \cap[0, m-1]$, it would suffice to set $\varphi_{r, q}(u) \equiv 0$ for $q \in \mathbb{Z} \backslash[0, r]$, and

$$
\left\{\begin{array}{l}
\varphi_{0,0}(u) \equiv 1, \\
\varphi_{r, q}(u)=\varphi_{r-1, q-1}(u)+\frac{D^{1}\left[\mathcal{L}_{m}(u) \varphi_{r-1, q}(u)\right]}{\mathcal{L}_{m}(u)}+\frac{(-1)^{r} \ell_{m, m-r}(u)}{\mathcal{L}_{m}(u)} \varphi_{0, q}(u) .
\end{array}\right.
$$

It is then not hard to verify that

$$
\varphi_{r, q}(u)=\sum_{p=m-r+q}^{m}(-1)^{p+1}\left(\begin{array}{c}
r+p-m \\
q
\end{array}\right) \frac{D^{r+p-m-q} \ell_{m, p}(u)}{\mathcal{L}_{m}(u)}
$$

for $q \in \mathbb{Z} \cap[0, r]$ is the unique solution to the aforementioned recursion. This explains the origin of (3.7).

Suppose that the Wrońskian matrix $\mathbf{W}_{m}(u)=\mathbf{W}\left[f_{1}(u), \ldots, f_{m}(u)\right]$ of our concern has determinant $\Lambda_{m}\left|\mathcal{L}_{m}(u)\right|^{-m / 2}$, where $\Lambda_{m} \neq 0$. The statement of Proposition 3.1 guarantees the existence of a constant matrix $\mathbf{S}_{m}$ such that the last column of $\mathbf{W}^{\star}=\mathbf{W}_{m}^{\star}(u)$ satisfies [cf. (2.36)]

$$
\frac{\left|\mathcal{L}_{m}(u)\right|^{\frac{m-2}{2}}}{\Lambda_{m}}\left(\left(\mathbf{W}^{\star}\right)_{1, m}, \ldots,\left(\mathbf{W}^{\star}\right)_{m, m}\right)^{\mathrm{T}}=\mathbf{S}_{m}\left(f_{1}(u), \ldots, f_{m}(u)\right)^{\mathrm{T}} .
$$


The result in Proposition 3.2 then tells us that $\mathbf{W}_{m}^{\star}(u)=\frac{(-1)^{m+1} \Lambda_{m}}{\left|\mathcal{L}_{m}(u)\right|^{(m-2) / 2}} \mathbf{S}_{m} \mathbf{W}_{m}^{\mathrm{T}}(u) \mathbf{V}_{m}^{\mathrm{T}}(u)$, where $\left|\operatorname{det} \mathbf{V}_{m}^{\mathrm{T}}(u)\right|=$ 1 , so the adjugate equation $\mathbf{W} \mathbf{W}^{\star}=\operatorname{det}(\mathbf{W}) \mathbf{I}$ for $\mathbf{W}=\mathbf{W}_{m}(u)$ and $\operatorname{det}(\mathbf{W})=\Lambda_{m}\left|\mathcal{L}_{m}(u)\right|^{-m / 2}$ translates into

$$
\mathbf{S}_{m}=(-1)^{m+1} \frac{\left[\mathbf{W}_{m}(u)\right]^{-1}\left[\mathbf{V}_{m}^{\mathrm{T}}(u)\right]^{-1}\left[\mathbf{W}_{m}^{\mathrm{T}}(u)\right]^{-1}}{\left|\mathcal{L}_{m}(u)\right|}
$$

Corollary 3.3 ((Skew) symmetries of certain matrices). Under the same assumptions as Proposition 3.2 we have the relation $\mathbf{V}_{m}(u)=(-1)^{m+1} \mathbf{V}_{m}^{\mathrm{T}}(u)$, which entails $\mathbf{S}_{m}=(-1)^{m+1} \mathbf{S}_{m}^{\mathrm{T}}$.

Proof. First we show that $\left(\mathbf{V}_{2 k-1}(u)\right)_{a, b}=\left(\mathbf{V}_{2 k-1}(u)\right)_{b, a}$ for $k \in \mathbb{Z}_{>0}$.

Along the main anti-diagonal, we have $\left(\mathbf{V}_{2 k-1}(u)\right)_{a, 2 k-a}=(-1)^{a-1}=\left(\mathbf{V}_{2 k-1}(u)\right)_{2 k-a, a}$ for $a \in \mathbb{Z} \cap[1,2 k-$ 1]. As we march away from the main anti-diagonal, and work with $a+b \in \mathbb{Z} \cap(2,2 k), a \in \mathbb{Z} \cap[1,2 k-$ $2], b \in \mathbb{Z} \cap[2,2 k-2]$, we have the following recursion:

$$
\begin{aligned}
\left(\mathbf{V}_{2 k-1}(u)\right)_{a, b}+\left(\mathbf{V}_{2 k-1}(u)\right)_{a+1, b-1} & =\sum_{n=a+b-1}^{2 k-1}(-1)^{a+n}\left[\left(\begin{array}{c}
n-a \\
b-1
\end{array}\right)-\left(\begin{array}{c}
n-a-1 \\
b-2
\end{array}\right)\right] \frac{D^{n-a-b+1} \ell_{2 k-1, n}(u)}{\mathcal{L}_{2 k-1}(u)} \\
& =\sum_{n=a+b}^{2 k-1}(-1)^{a+n}\left(\begin{array}{c}
n-a-1 \\
b-1
\end{array}\right) \frac{D^{n-a-b+1} \ell_{2 k-1, n}(u)}{\mathcal{L}_{2 k-1}(u)} \\
& =-\frac{D^{1}\left[\mathcal{L}_{2 k-1}(u)\left(\mathbf{V}_{2 k-1}(u)\right)_{a+1, b}\right]}{\mathcal{L}_{2 k-1}(u)}
\end{aligned}
$$

In view of this recursion, in order to show that $\left(\mathbf{V}_{2 k-1}(u)\right)_{a, 2 k-1-a}=\left(\mathbf{V}_{2 k-1}(u)\right)_{2 k-1-a, a}$ for all $a \in$ $\mathbb{Z} \cap[1,2 k-2]$, it would suffice to verify that $\left(\mathbf{V}_{2 k-1}(u)\right)_{1,2 k-2}=\left(\mathbf{V}_{2 k-1}(u)\right)_{2 k-2,1}$. Direct computations reveal that

$$
\begin{aligned}
\left(\mathbf{V}_{2 k-1}(u)\right)_{1,2 k-2}-\left(\mathbf{V}_{2 k-1}(u)\right)_{2 k-2,1} & =\sum_{n=2 k-2}^{2 k-1}(-1)^{1+n}\left[\left(\begin{array}{c}
n-1 \\
2 k-3
\end{array}\right)+\left(\begin{array}{c}
n-2 k+2 \\
0
\end{array}\right)\right] \frac{D^{n-2 k+2} \ell_{2 k-1, n}(u)}{\mathcal{L}_{2 k-1}(u)} \\
& =\frac{1}{\mathcal{L}_{2 k-1}(u)}\left[(2 k-1) D^{1} \ell_{2 k-1,2 k-1}(u)-2 \ell_{2 k-1,2 k-2}(u)\right]=0
\end{aligned}
$$

according to (2.13).

The efforts in the last paragraph amount to a verification that $\left(\mathbf{V}_{2 k-1}(u)\right)_{a, b}=\left(\mathbf{V}_{2 k-1}(u)\right)_{b, a}$ holds for $a+b=2 k-1$. To show that the same identity holds true for smaller values of $a+b$, we exploit (3.17) again, and build up on the "boundary conditions" $\left(\mathbf{V}_{2 k-1}(u)\right)_{1, b}=\left(\mathbf{V}_{2 k-1}(u)\right)_{b, 1}$ for $b \in \mathbb{Z} \cap[2,2 k-3]$. By (3.7) and elementary combinatorics, we have

$$
\begin{aligned}
\left(\mathbf{V}_{2 k-1}(u)\right)_{1, b}-\left(\mathbf{V}_{2 k-1}(u)\right)_{b, 1} & =\sum_{n=b}^{2 k-1}\left[(-1)^{1+n}\left(\begin{array}{l}
n-1 \\
b-1
\end{array}\right)-(-1)^{b+n}\left(\begin{array}{c}
n-b \\
0
\end{array}\right)\right] \frac{D^{n-b} \ell_{2 k-1, n}(u)}{\mathcal{L}_{2 k-1}(u)} \\
& =\sum_{n=b}^{2 k-1}\left[(-1)^{1+n}\left(\begin{array}{l}
n \\
b
\end{array}\right)+(-1)^{n}\left(\begin{array}{c}
n-1 \\
b
\end{array}\right)-(-1)^{b+n}\right] \frac{D^{n-b} \ell_{2 k-1, n}(u)}{\mathcal{L}_{2 k-1}(u)} .
\end{aligned}
$$

Referring back to the combinatorial constraint in (2.12), which says

$$
-\ell_{2 k-1, b}(u)=\sum_{n=b}^{2 k-1}(-1)^{n}\left(\begin{array}{l}
n \\
b
\end{array}\right) D^{n-b} \ell_{2 k-1, n}(u),
$$


we may further reduce (3.19) into

$$
\begin{aligned}
& \left(\mathbf{V}_{2 k-1}(u)\right)_{1, b}-\left(\mathbf{V}_{2 k-1}(u)\right)_{b, 1} \\
= & \frac{\ell_{2 k-1, b}(u)-\ell_{2 k-1, b}(u)}{\mathcal{L}_{2 k-1}(u)}-\sum_{n=b+1}^{2 k-1}\left[(-1)^{1+n}\left(\begin{array}{c}
n-1 \\
b+1-1
\end{array}\right)-(-1)^{b+1+n}\right] \frac{D^{n-b-1+1} \ell_{2 k-1, n}(u)}{\mathcal{L}_{2 k-1}(u)} \\
= & -\frac{D^{1}\left\{\mathcal{L}_{2 k-1}(u)\left[\left(\mathbf{V}_{2 k-1}(u)\right)_{1, b+1}-\left(\mathbf{V}_{2 k-1}(u)\right)_{b+1,1}\right]\right\}}{\mathcal{L}_{2 k-1}(u)} .
\end{aligned}
$$

Thus, it is clear that $\left(\mathbf{V}_{2 k-1}(u)\right)_{1, b}=\left(\mathbf{V}_{2 k-1}(u)\right)_{b, 1}$ indeed holds for $b \in \mathbb{Z} \cap[2,2 k-3]$, so $\mathbf{V}_{2 k-1}(u)=$ $\mathbf{V}_{2 k-1}^{\mathrm{T}}(u)$ is true.

Since $\left[\mathbf{W}_{2 k-1}^{\mathrm{T}}(u)\right]^{-1}$ is the transpose of $\left[\mathbf{W}_{2 k-1}(u)\right]^{-1}$, the relation $(3.16)$ allows us to deduce $\mathbf{S}_{2 k-1}=$ $\mathbf{S}_{2 k-1}^{\mathrm{T}}$ from $\mathbf{V}_{2 k-1}(u)=\mathbf{V}_{2 k-1}^{\mathrm{T}}(u)$.

The aforementioned techniques also lead us to $\left(\mathbf{V}_{2 k}(u)\right)_{1, b}=-\left(\mathbf{V}_{2 k}(u)\right)_{b, 1}$ for $b \in \mathbb{Z} \cap[2,2 k]$, and consequently $\left(\mathbf{V}_{2 k}(u)\right)_{a, b}=-\left(\mathbf{V}_{2 k}(u)\right)_{b, a}$ for $a+b \in \mathbb{Z} \cap[3,2 k+1]$. What remains to be investigated is the case where $a=b=1$. By direct computation, we have

$$
\begin{aligned}
& \left(\mathbf{V}_{2 k}(u)\right)_{1,1}=\sum_{n=1}^{2 k}(-1)^{n}\left(\begin{array}{c}
n-1 \\
0
\end{array}\right) \frac{D^{n-1} \ell_{2 k, n}(u)}{\mathcal{L}_{2 k}(u)}=\sum_{n=1}^{2 k}(-1)^{n} \frac{D^{n-1} \ell_{2 k, n}(u)}{\mathcal{L}_{2 k}(u)} \\
& \left(\mathbf{V}_{2 k}(u)\right)_{2,1}=\sum_{n=2}^{2 k}(-1)^{n+1}\left(\begin{array}{c}
n-2 \\
0
\end{array}\right) \frac{D^{n-2} \ell_{2 k, n}(u)}{\mathcal{L}_{2 k}(u)}=\sum_{n=2}^{2 k}(-1)^{n+1} \frac{D^{n-2} \ell_{2 k, n}(u)}{\mathcal{L}_{2 k}(u)} \\
& \left(\mathbf{V}_{2 k}(u)\right)_{1,2}=\sum_{n=2}^{2 k}(-1)^{n}\left(\begin{array}{c}
n-1 \\
1
\end{array}\right) \frac{D^{n-2} \ell_{2 k, n}(u)}{\mathcal{L}_{2 k}(u)}=\sum_{n=2}^{2 k}(-1)^{n}(n-1) \frac{D^{n-2} \ell_{2 k, n}(u)}{\mathcal{L}_{2 k}(u)}
\end{aligned}
$$

so another invocation of (2.12) brings us

$$
\begin{aligned}
2\left(\mathbf{V}_{2 k}(u)\right)_{1,1} & =2\left(\mathbf{V}_{2 k}(u)\right)_{1,1}+\frac{D^{1}\left\{\mathcal{L}_{2 k}(u)\left[\left(\mathbf{V}_{2 k}(u)\right)_{2,1}+\left(\mathbf{V}_{2 k}(u)\right)_{1,2}\right]\right\}}{\mathcal{L}_{2 k}(u)} \\
& =-\frac{2 \ell_{2 k, 1}(u)}{\mathcal{L}_{2 k}(u)}+\sum_{n=2}^{2 k}(-1)^{n}\left(\begin{array}{c}
n \\
1
\end{array}\right) \frac{D^{n-1} \ell_{2 k, n}(u)}{\mathcal{L}_{2 k}(u)} \\
& =\frac{-2 \ell_{2 k, 1}(u)+\ell_{2 k, 1}(u)+\ell_{2 k, 1}(u)}{\mathcal{L}_{2 k}(u)}=0,
\end{aligned}
$$

as expected.

Now that we have $\mathbf{V}_{2 k}(u)=-\mathbf{V}_{2 k}^{\mathrm{T}}(u)$, the skew symmetry $\mathbf{S}_{2 k}=-\mathbf{S}_{2 k}^{\mathrm{T}}$ follows from (3.16).

3.2. Threshold behavior of Wrońskian cofactors. Naturally, our next goal is to seek explicit formulae (in terms of fundamental solutions to Vanhove's differential equations) for the cofactor Wrońskian determinants

$$
\begin{aligned}
(-1)^{r+m}\left(\mathbf{W}_{m}^{\star}(u)\right)_{r, m} & =W\left[\mathcal{F}_{m, 1}(u), \ldots, \widehat{\mathcal{F}_{m, r}(u)}, \ldots, \mathcal{F}_{m, m}(u)\right] \\
& =\operatorname{det}\left(D^{i-1} \mathcal{F}_{m, j}(u)\right)_{i \in \mathbb{Z} \cap[1, m-1], j \in \mathbb{Z} \cap([1, r-1] \cup[r+1, m])},
\end{aligned}
$$

for all $r \in \mathbb{Z} \cap[1, m]$. This will amount to the complete characterizations of the matrix $\mathbf{S}_{m}$ that fits into the identity (2.36) for $u \in(0,1)$.

Even though the (skew) symmetry relations in Corollary 3.3 may nearly halve our workload on the elements of $\mathbf{S}_{m}$, we still need somewhat extensive preparations in Lemmata 3.4 and 3.5 below, before carrying out our computations for $k \in \mathbb{Z}_{>2}$ in Proposition 3.6. 
Let $C^{\omega}(a, b)$ be the totality of real-analytic functions 10 on the interval $(a, b)$. The following patterns

$$
\begin{gathered}
\frac{\left|\mathcal{L}_{2 k-1}(u)\right|^{k-\frac{3}{2}}}{|4-u|^{k-\frac{3}{2}}} W\left[\mathcal{F}_{2 k-1,1}(u), \ldots, \overline{\mathcal{F}_{2 k-1, k}(u)}, \ldots, \mathcal{F}_{2 k-1,2 k-1}(u)\right] \in C^{\omega}(0,16), \\
\frac{\left|\mathcal{L}_{2 k}(u)\right|^{k-1}}{|1-u|^{k-1}} W\left[\mathcal{F}_{2 k, 1}(u), \ldots, \overline{\mathcal{F}_{2 k, k+1}(u)}, \ldots, \mathcal{F}_{2 k, 2 k}(u)\right] \in C^{\omega}(0,9)
\end{gathered}
$$

motivate us to search for certain linear combinations of Bessel moments with suitable orders of vanishing and analytic behavior as $u$ approaches a threshold, in the next lemma.

Lemma 3.4 (Local behavior near thresholds). In what follows, the exact value of a positive number $\varepsilon$ may vary from context to context.

(a) For each $s \in \mathbb{Z} \cap[1, k]$, there exists a real-analytic function $\mathrm{f}_{k, s} \in C^{\omega}\left((2 s)^{2}-\varepsilon,(2 s)^{2}+\varepsilon\right)$ such that we have

$$
\operatorname{ReF}_{k, s}^{1}(u)=\frac{(-4 s)^{1-k}}{(2 k-3) ! !} \frac{(-1)^{\left\lfloor\frac{k-s}{2}\right\rfloor+(k-1)(k-s)}}{2}\left|u-(2 s)^{2}\right|^{k-\frac{3}{2}}\left\{1+\left[u-(2 s)^{2}\right] f_{k, s}(u)\right\},
$$

for $\left((2 s)^{2}-u\right)(-1)^{k-s} \in[0, \varepsilon)$, and

$$
\operatorname{ImF}_{k, s}^{1}(u)=\frac{(-4 s)^{1-k}}{(2 k-3) ! !} \frac{(-1)^{\left\lfloor\frac{k-s-1}{2}\right\rfloor+(k-1)(k-s-1)}}{2}\left|u-(2 s)^{2}\right|^{k-\frac{3}{2}}\left\{1+\left[u-(2 s)^{2}\right] \mathrm{f}_{k, s}(u)\right\},
$$

for $\left((2 s)^{2}-u\right)(-1)^{k-s-1} \in[0, \varepsilon)$.

(b) For each $s \in \mathbb{Z} \cap[1, k+1]$, there exists a real-analytic function $\mathrm{g}_{k, s} \in C^{\omega}\left((2 s-1)^{2}-\varepsilon,(2 s-1)^{2}+\varepsilon\right)$ such that we have

$$
\begin{aligned}
\tilde{\mathfrak{b}}_{k, s}(u) & :=\frac{(-1)^{\left\lfloor\frac{k-s}{2}\right\rfloor+k-s}}{2^{2 k}(k-1) !} \sqrt{\frac{\pi}{(2 s-1)^{2 k-1}}}\left[u-(2 s-1)^{2}\right]^{k-1}\left\{1+\left[u-(2 s-1)^{2}\right] \mathrm{g}_{k, s}(u)\right\} \\
& = \begin{cases}\operatorname{ReG}_{k, s}^{1}(u), & (-1)^{k-s}=+1, \\
\operatorname{ImG}_{k, s}^{1}(u), & (-1)^{k-s}=-1,\end{cases}
\end{aligned}
$$

and the expression

$$
\begin{aligned}
& \mathfrak{Q}_{k, s}(u) \\
:= & \begin{cases}\mathcal{I}_{2 k, s}^{1}(u)+\frac{(-1)^{k-s-1}-i}{\pi(1-i)} \mathfrak{5}_{k, s}(u)\left[\log \left|u-(2 s-1)^{2}\right|-\frac{i \pi}{2}\right], & u \in\left((2 s-1)^{2}-\varepsilon,(2 s-1)^{2}\right), \\
\mathcal{K}_{2 k, s}^{1}(u)+\frac{(-1)^{k-s-1}-i}{\pi(1-i)}\left(\mathfrak{b}_{k, s}(u)\left[\log \left|u-(2 s-1)^{2}\right|+\frac{i \pi}{2}\right],\right. & u \in\left((2 s-1)^{2},(2 s-1)^{2}+\varepsilon\right)\end{cases}
\end{aligned}
$$

extends to a real-analytic function in $C^{\omega}\left((2 s-1)^{2}-\varepsilon,(2 s-1)^{2}+\varepsilon\right)$.

Proof. (a) If $k-s$ is even, then $\operatorname{ReF}_{k, s}^{1}(u)$ [see (2.38) and (2.39) for explicit formulae] is well-defined and real-analytic for $u \in\left((2 s-2)^{2},(2 s)^{2}\right)$, and the function $(-1)^{\left\lfloor\frac{k-s}{2}\right\rfloor}\left(1+2 k \delta_{s, k}\right) \mathcal{F}_{2 k-1,2\left\lfloor\frac{k-s}{2}\right\rfloor+1}(u)=$ $(-1)^{\left\lfloor\frac{k-s}{2}\right\rfloor}\left(1+2 k \delta_{s, k}\right) \mathcal{F}_{2 k-1, k-s+1}(u)$ is the only addend that fails to be real-analytic in a neighborhood of $u=(2 s)^{2}$. Moreover, the function $\operatorname{Re}_{k, s}^{1}(u)$ has vanishing derivatives up to order $k-2=$ $\max \left\{2\left(\left\lfloor\frac{k}{2}\right\rfloor-1\right), 2\left\lfloor\frac{k-1}{2}\right\rfloor-1\right\}$, according to Proposition 2.9(a). We claim that the leading order behavior for $\operatorname{ReF}_{k, s}^{1}(u)$ is $A\left[(2 s)^{2}-u\right]^{k-\frac{3}{2}}[1+o(1)]$, where the constant $A$ can be determined through

\footnotetext{
${ }^{10}$ For each $u_{0} \in(a, b)$, there exists a positive number $\varepsilon$, such that the Taylor series $\sum_{n=0}^{\infty} \frac{\left(u-u_{0}\right)^{n}}{n !} D^{n} f\left(u_{0}\right)$ of a real-analytic function $f \in C^{\omega}(a, b)$ converges to $f(u)$ for all $u \in\left(u_{0}-\varepsilon, u_{0}+\varepsilon\right)$. As a slight abuse of notations, we sometimes use the same symbol for a real-analytic function and its real-analytic continuation to a larger domain of definition.
} 
l'Hôpital's rule. Concretely speaking, when $k$ is odd, we have

$$
\begin{aligned}
A & =\lim _{u \rightarrow(2 s)^{2}-0^{+}} \frac{\operatorname{ReF}_{k, s}^{1}(u)}{\left[(2 s)^{2}-u\right]^{k-\frac{3}{2}}}=\lim _{u \rightarrow(2 s)^{2}-0^{+}} \frac{D^{k-1} \operatorname{ReF}_{k, s}^{1}(u)}{D^{k-1}\left\{\left[(2 s)^{2}-u\right]^{k-\frac{3}{2}}\right\}} \\
& =\frac{\left(1+2 k \delta_{s, k}\right)(-1)^{\left\lfloor\frac{k-s}{2}\right\rfloor}}{(-2)^{1-k}(2 k-3) ! !} \lim _{u \rightarrow(2 s)^{2}-0^{+}} \sqrt{(2 s)^{2}-u} D^{k-1} \mathcal{F}_{2 k-1, k-s+1}(u),
\end{aligned}
$$

where

$$
\begin{aligned}
& \lim _{u \rightarrow(2 s)^{2}-0^{+}} \sqrt{(2 s)^{2}-u} D^{k-1} \mathcal{F}_{2 k-1, k-s+1}(u) \\
= & \frac{1}{(4 s)^{k-1}} \lim _{u \rightarrow(2 s)^{2}-0^{+}} \sqrt{(2 s)^{2}-u} \mathcal{F}_{2 k-1, k-s+1}^{\frac{k+1}{2}}(u) \\
= & \frac{1}{(4 s)^{k-1}} \lim _{u \rightarrow(2 s)^{2}-0^{+}} \frac{\sqrt{(2 s)^{2}-u}}{\pi^{s}\left(1+2 k \delta_{s, k}\right)} \int_{0}^{\infty} \frac{e^{\sqrt{u} t}}{\sqrt{2 \pi \sqrt{u} t}}\left(\frac{e^{t}}{\sqrt{2 \pi t}}\right)^{k-s}\left(\sqrt{\frac{\pi}{2 t}} e^{-t}\right)^{k+s} t^{k} \mathrm{~d} t \\
= & \frac{2^{2-3 k} s^{1-k}}{1+2 k \delta_{s, k}}
\end{aligned}
$$

follows from the asymptotic behavior [46, §7.23]

$$
I_{0}(t)=\frac{e^{t}}{\sqrt{2 \pi t}}\left[1+O\left(\frac{1}{t}\right)\right] \quad \text { and } \quad K_{0}(t)=\sqrt{\frac{\pi}{2 t}} e^{-t}\left[1+O\left(\frac{1}{t}\right)\right],
$$

along with Bessel's differential equations $\left(u D^{2}+D^{1}\right) I_{0}(t)=\frac{t^{2}}{4} I_{0}(t)$ and $\left(u D^{2}+D^{1}\right) K_{0}(t)=\frac{t^{2}}{4} K_{0}(t)$. This shows that $A=\frac{(-4 s)^{1-k}}{(2 k-3) ! !} \frac{(-1)^{\left\lfloor\frac{k-s}{2}\right\rfloor}}{2}$ when $k$ is odd. Based on the asymptotic behavior [46, §7.23]

$$
I_{1}(t)=\frac{e^{t}}{\sqrt{2 \pi t}}\left[1+O\left(\frac{1}{t}\right)\right] \text { and } K_{1}(t)=\sqrt{\frac{\pi}{2 t}} e^{-t}\left[1+O\left(\frac{1}{t}\right)\right],
$$

one can perform a similar analysis when $k$ is even.

If $k-s$ is odd, then $\operatorname{ReF}_{k, s}^{1}(u)$ is well-defined and real-analytic for $u \in\left((2 s)^{2},(2 s+2)^{2}\right)$, and the function $(-1)^{\left\lfloor\frac{k+s-1}{2}\right\rfloor} \mathcal{F}_{2 k-1,2\left\lfloor\frac{k+s-1}{2}\right\rfloor+k}^{\ell}(u)=(-1)^{\left\lfloor\frac{k+s-1}{2}\right\rfloor} \mathcal{F}_{2 k-1,3 k+s-1}^{\ell}(u)$ is the only addend that fails to be real-analytic in a neighborhood of $u=(2 s)^{2}$. One can repeat the remaining procedures in the last paragraph to find the leading order behavior in such scenarios.

By the Fuchs condition [31, §15.3], the operator $\widetilde{L}_{2 k-1}$ has a regular singular point at $u=(2 s)^{2}$ for each $s \in \mathbb{Z} \cap[1, k]$, so the left-hand sides of (3.28) and (3.29) should be equal to convergent generalized power series. According to the Frobenius method [31, §16.1], such series may (in principle) involve both integer powers $\left[u-(2 s)^{2}\right]^{\mathbb{Z}}$ and half-integer powers $\left|u-(2 s)^{2}\right|^{\mathbb{Z}+\frac{1}{2}}$. Our next task is to show that integer powers $\left[u-(2 s)^{2}\right]^{\mathbb{Z}}$ actually will not appear in these scenarios.

Without loss of generality, we only elaborate on the cases where $u<(2 s)^{2}, k-s$ is even, and $k$ is odd. We start from the following asymptotic expansions for $M \in \mathbb{Z}_{\geq 0}, t \rightarrow \infty$ :

$$
\begin{aligned}
\mathscr{F}_{k, s, M}(u, t) t^{k+2 M}:= & \left\{I_{0}(\sqrt{u} t)\left[-i K_{0}(t)\right]^{k+s} \frac{\left[\pi I_{0}(t)+i K_{0}(t)\right]^{k-s}+\left[\pi I_{0}(t)-i K_{0}(t)\right]^{k-s}}{2}\right. \\
& +i K_{0}(\sqrt{u} t)\left[-i K_{0}(t)\right]^{k+s} \frac{\left[\pi I_{0}(t)+i K_{0}(t)\right]^{k-s}-\left[\pi I_{0}(t)-i K_{0}(t)\right]^{k-s}}{2} \\
& \left.+i K_{0}(\sqrt{u} t)\left[-i K_{0}(t)\right]^{k-s} \frac{\left[\pi I_{0}(t)+i K_{0}(t)\right]^{k+s}-\left[\pi I_{0}(t)-i K_{0}(t)\right]^{k+s}}{2}\right\} \frac{t^{k+2 M}}{\pi^{k+1}}
\end{aligned}
$$




$$
-\frac{e^{(\sqrt{u}-2 s) t}}{\sqrt{t}} \sum_{n=0}^{2 M} \frac{f_{k, s, n}(u)}{t^{n-2 M}}=O\left(\frac{e^{(\sqrt{u}-2 s) t}}{|t|^{3 / 2}}\right)
$$

where $f_{k, s, n}(u)$ is holomorphic in a $\mathbb{C}$-neighborhood of $u=(2 s)^{2}$. Integrating the left-hand side of the equation above over $t \in(0, \infty)$, while invoking the dominated convergence theorem, we obtain

$$
\lim _{u \rightarrow(2 s)^{2}-0^{+}}\left[\operatorname{ReF}_{k, s}^{\frac{k}{2}+M}(u)-\sum_{n=0}^{2 M} \frac{f_{k, s, n}(u) \Gamma\left(2 M-n+\frac{1}{2}\right)}{|\sqrt{u}-2 s|^{2 M-n+\frac{1}{2}}}\right]=\int_{0}^{\infty} \mathscr{F}_{k, s, M}\left((2 s)^{2}, t\right) t^{k+2 M} \mathrm{~d} t .
$$

The right-hand side of the equation above vanishes, because it is a constant multiple of [cf. (2.42)]

$$
\operatorname{Re} \int_{-i \infty}^{i \infty}\left\{H_{0}^{(2)}(2 s z)\left[H_{0}^{(1)}(z)\right]^{2 s}\left[H_{0}^{(1)}(z) H_{0}^{(2)}(z)\right]^{k-s} z^{k+2 M}-\frac{1}{\sqrt{z}} \sum_{n=0}^{2 M} \frac{h_{k, s, n}}{z^{n-2 M}}\right\} \mathrm{d} z,
$$

where the integrand has $O\left(|z|^{-3 / 2}\right.$ ) asymptotics for $|z| \rightarrow \infty,|\arg z| \leq \frac{\pi}{2}$, and the contour closes to the right. So far, we know that the generalized power series expansion for $\operatorname{Re}_{k, s}^{\frac{k}{2}+M}(u)$ contains no $[\sqrt{u}-2 s]^{\mathbb{Z}_{\leq 0}}$ terms, for each $M \in \mathbb{Z}_{\geq 0}$. Likewise, one can argue that the same property applies to $\operatorname{Re}^{\frac{k}{2}+M}(u), M \in \mathbb{Z}_{\geq 0}$. This proves that the generalized power series expansion for $\mathrm{F}_{k, s}^{1}(u)$ contains no $[\sqrt{u}-2 s]^{\mathbb{Z}}$ terms, and equivalently, no $\left[u-(2 s)^{2}\right]^{\mathbb{Z}}$ terms, as claimed.

(b) Without loss of generality, we will focus on the scenarios where $k-s$ is even, and $k$ is odd. Under such assumptions, one can show (through direct examination of the integral representations for offshell Bessel moments) that $\operatorname{Re}_{k, s}^{1}(u)$ is real-analytic for $u \in\left((\max \{0,2 s-3\})^{2},(2 s+1)^{2}\right)$. At the threshold $u=(2 s-1)^{2}$, this function has vanishing derivatives up to $(k-2)$ nd order, according to Proposition 2.9(b).

As a variation on our proof [53, Lemma 2] of the integral formulae for generalized Crandall numbers 2.47), we consider

$$
\left(\int_{-i T}^{-i \varepsilon}+\int_{C_{\varepsilon}}+\int_{i \varepsilon}^{i T}\right)\left\{\frac{H_{0}^{(2)}((2 s-1) z)}{(2 / \pi)^{k+1}}\left[H_{0}^{(1)}(z)\right]^{2 s-1}\left[H_{0}^{(1)}(z) H_{0}^{(2)}(z)\right]^{k+1-s} z^{k}-\frac{(-1)^{\left\lfloor\frac{s}{2}\right\rfloor}}{\sqrt{2 s-1} z}\right\} \mathrm{d} z
$$

where $C_{\varepsilon}$ is a semi-circular arc in the right half-plane, joining $-i \varepsilon$ to $i \varepsilon$. For each fixed $\varepsilon>0$, we can close the contour to the right, as $T \rightarrow \infty$. Noting that $\int_{C_{\varepsilon}} \frac{\mathrm{d} z}{z}=\pi i$, we may read off the imaginary part of the last displayed equation as $\operatorname{Re}_{k, s}^{\frac{k+1}{2}}\left((2 s-1)^{2}\right)=\frac{(-1)^{\left\lfloor\frac{s}{2}\right\rfloor}}{2^{k+1}} \sqrt{\frac{\pi}{2 s-1}}$. Falling back on l'Hôpital's rule, we can deduce $\operatorname{Re}_{k, s}^{1}(u)=\frac{(-1)^{\left.\frac{k-s}{2}\right\rfloor}}{2^{2 k}(k-1) !} \sqrt{\frac{\pi}{(2 s-1)^{2 k-1}}}\left[u-(2 s-1)^{2}\right]^{k-1}[1+o(1)]$ as $u \rightarrow(2 s-1)^{2}$. Here, the $o(1)$ term can be rewritten as $\left[u-(2 s-1)^{2}\right] \mathrm{g}_{k, s}(u)$ with $\mathrm{g}_{k, s} \in C^{\omega}\left((2 s-1)^{2}-\varepsilon,(2 s-1)^{2}+\varepsilon\right)$, because $\operatorname{Re}_{k, s}^{1}(u)$ is real-analytic as $u$ approaches $(2 s-1)^{2}$.

One can represent the Taylor coefficients $g_{k, s, n}=n ! D^{n} \mathrm{~g}_{k, s}\left((2 s-1)^{2}\right), n \in \mathbb{Z}_{\geq 0}$ through the offshell moments $\operatorname{ReG}_{k, s}^{\frac{k+1}{2}+M}\left((2 s-1)^{2}\right)$ and $\operatorname{Re}_{k, s}^{\frac{k+1}{2}+M}\left((2 s-1)^{2}\right)$ for $M \in \mathbb{Z}_{\geq 0}$. Here, one can evaluate $\operatorname{Re} G_{k, s}^{\frac{k+1}{2}+M}\left((2 s-1)^{2}\right)$ via a contour integral over

$$
\frac{H_{0}^{(2)}((2 s-1) z)}{(2 / \pi)^{k+1}}\left[H_{0}^{(1)}(z)\right]^{2 s-1}\left[H_{0}^{(1)}(z) H_{0}^{(2)}(z)\right]^{k+1-s} z^{k+2 M}-\sum_{n=1}^{2 M+1} \frac{\eta_{k, s, n}}{z^{n-2 M}}=O\left(\frac{1}{z^{2}}\right),
$$

akin to our treatment of (3.39). It then follows that $\operatorname{Re}_{k, s}^{\frac{k+1}{2}+M}\left((2 s-1)^{2}\right)=\frac{\sqrt{\pi}}{2^{k+1}}(-1)^{M} \eta_{k, s, 2 M+1}$. There is a similar relation that expresses $\operatorname{Re}_{k, s}^{\frac{k+1}{2}+M}\left((2 s-1)^{2}\right)$ through coefficients of asymptotic expansions. 
Now, for $M \in \mathbb{Z}_{\geq 0}$, we consider

$$
\begin{aligned}
\mathscr{H}_{k, s, M}(u, z) z^{k+2 M}:= & \frac{H_{0}^{(2)}(\sqrt{u} z)}{(2 / \pi)^{k+1}}\left[H_{0}^{(1)}(z)\right]^{2 s-1}\left[H_{0}^{(1)}(z) H_{0}^{(2)}(z)\right]^{k+1-s} z^{k+2 M} \\
& -e^{-i[\sqrt{u}-(2 s-1)] z}\left[\frac{\eta_{k, s, 2 M+1}(u)}{z+1}+\sum_{n=1}^{2 M} \frac{\eta_{k, s, n}(u)}{z^{n-2 M}}\right],
\end{aligned}
$$

where the functions $\eta_{k, s, n}(u)$ are holomorphic in a $\mathbb{C}$-neighborhood of $u=(2 s-1)^{2}$, and $\mathscr{H}_{k, s, M}(u, z)=$ $O\left(e^{-i[\sqrt{u}-(2 s-1)] z} / z^{2-k-2 M}\right)$ for fixed $u$ and $|z| \rightarrow \infty,-\frac{\pi}{2} \leq \arg z \leq \frac{\pi}{2}$. For $u \in\left((2 s-1)^{2}-\varepsilon,(2 s-1)^{2}\right)$, we can invoke Jordan's lemma in the form of

$$
\int_{0}^{i \infty} \mathscr{H}_{k, s, M}(u, z) z^{k+2 M} \mathrm{~d} z=\int_{0}^{\infty} \mathscr{H}_{k, s, M}(u, x) x^{k+2 M} \mathrm{~d} x .
$$

In view of (2.41), we rewrite the left-hand side of (3.42) as

$$
\begin{aligned}
& \left(\frac{2}{\pi i}\right)^{k+1}(-1)^{M} \int_{0}^{\infty}\left[i \pi I_{0}(\sqrt{u} t)-K_{0}(\sqrt{u} t)\right]\left[K_{0}(t)\right]^{k+s}\left[i \pi I_{0}(t)-K_{0}(t)\right]^{k+1-s} t^{k+2 M} \mathrm{~d} t \\
& -\eta_{k, s, 2 M+1}(u) \int_{0}^{i \infty} \frac{e^{-i[\sqrt{u}-(2 s-1)] z}}{z+1} \mathrm{~d} z-\sum_{n=1}^{2 M} \frac{\eta_{k, s, n}(u)(2 M-n) !}{\{i[\sqrt{u}-(2 s-1)]\}^{2 M+1-n}},
\end{aligned}
$$

where $\eta_{k, s, 2 M+1}\left((2 s-1)^{2}\right)=\frac{2^{k+1}}{\sqrt{\pi}}(-1)^{M} \operatorname{Re}_{k, s}^{\frac{k+1}{2}+M}\left((2 s-1)^{2}\right)$ and

$$
\int_{0}^{i \infty} \frac{e^{-i[\sqrt{u}-(2 s-1)] z}}{z+1} \mathrm{~d} z=-\log |2 s-1-\sqrt{u}|-\gamma_{0}+\frac{i \pi}{2}+o(1)
$$

as $u \rightarrow(2 s-1)^{2}-0^{+}$. Meanwhile, the right-hand side of (3.42) is continuous across the threshold $u=(2 s-1)^{2}$, and admits analytic continuation to $u \in\left((2 s-1)^{2},(2 s-1)^{2}+\varepsilon\right)$, in the following form:

$$
\begin{aligned}
& \int_{0}^{\infty} \mathscr{H}_{k, s, M}(u, x) x^{k+2 M} \mathrm{~d} x=\int_{0}^{-i \infty} \mathscr{H}_{k, s, M}(u, z) z^{k+2 M} \mathrm{~d} z \\
= & \left(\frac{2}{\pi i}\right)^{k+1}(-1)^{M} \int_{0}^{\infty} K_{0}(\sqrt{u} t)\left[K_{0}(t)\right]^{k+1-s}\left[i \pi I_{0}(t)+K_{0}(t)\right]^{k+s} t^{k+2 M} \mathrm{~d} t \\
& -\eta_{k, s, 2 M+1}(u) \int_{0}^{-i \infty} \frac{e^{-i[\sqrt{u}-(2 s-1)] z}}{z+1} \mathrm{~d} z-\sum_{n=1}^{2 M} \frac{\eta_{k, s, n}(u)(2 M-n) !}{\{i[\sqrt{u}-(2 s-1)]\}^{2 M+1-n}},
\end{aligned}
$$

where

$$
\int_{0}^{-i \infty} \frac{e^{-i[\sqrt{u}-(2 s-1)] z}}{z+1} \mathrm{~d} z=-\log |2 s-1-\sqrt{u}|-\gamma_{0}-\frac{i \pi}{2}+o(1)
$$

as $u \rightarrow(2 s-1)^{2}+0^{+}$. Therefore, with $\mathrm{h}_{k, s}^{M}(u):=\sum_{n=1}^{2 M} \eta_{k, s, n}(u)(2 M-n) !\{i[\sqrt{u}-(2 s-1)]\}^{n-1} \in C^{\omega}((2 s-$ $\left.1)^{2}-\varepsilon,(2 s-1)^{2}+\varepsilon\right)$ and the understanding that $h_{k, s}^{0}(u) \equiv 0$, we have the following continuity condition for every $M \in \mathbb{Z}_{\geq 0}$ :

$$
\begin{aligned}
& \lim _{u \rightarrow(2 s-1)^{2}-0^{+}}\left\{\int_{0}^{\infty}\left[i \pi I_{0}(\sqrt{u} t)-K_{0}(\sqrt{u} t)\right]\left[K_{0}(t)\right]^{k+s}\left[i \pi I_{0}(t)-K_{0}(t)\right]^{k+1-s} t^{k+2 M} \mathrm{~d} t+\right. \\
&\left.+\left(\frac{\pi i}{2}\right)^{k+1}\left[\frac{2^{k+1}}{\sqrt{\pi}}\left(\log |2 s-1-\sqrt{u}|-\frac{i \pi}{2}\right) \operatorname{Re}_{k, s}^{\frac{k+1}{2}+M}\left((2 s-1)^{2}\right)-\frac{\mathrm{h}_{k, s}^{M}(u)}{[\sqrt{u}-(2 s-1)]^{2 M}}\right]\right\} \\
&=\lim _{u \rightarrow(2 s-1)^{2}+0^{+}}\left\{\int_{0}^{\infty} K_{0}(\sqrt{u} t)\left[K_{0}(t)\right]^{k+1-s}\left[i \pi I_{0}(t)+K_{0}(t)\right]^{k+s} t^{k+2 M} \mathrm{~d} t+\right.
\end{aligned}
$$




$$
\left.+\left(\frac{\pi i}{2}\right)^{k+1}\left[\frac{2^{k+1}}{\sqrt{\pi}}\left(\log |2 s-1-\sqrt{u}|+\frac{i \pi}{2}\right) \operatorname{ReG}_{k, s}^{\frac{k+1}{2}+M}\left((2 s-1)^{2}\right)-\frac{\mathrm{h}_{k, s}^{M}(u)}{[\sqrt{u}-(2 s-1)]^{2 M}}\right]\right\} .
$$

There is a similar continuity condition relating derivatives of these off-shell Bessel moments (with respect to $u$ ) to $\operatorname{Re}_{k, s}^{\frac{k+1}{2}+M}\left((2 s-1)^{2}\right)$.

Combining the input from the last two paragraphs, one sees that both $\mathcal{I}_{2 k, s}^{1}(u)-\frac{i}{\pi}\left(\mathfrak{b}_{k, s}(u)[\log \mid u-\right.$ $\left.(2 s-1)^{2} \mid-\frac{i \pi}{2}\right], u \in\left((2 s-1)^{2}-\varepsilon,(2 s-1)^{2}\right)$ and $\mathcal{K}_{2 k, s}^{1}(u)-\frac{i}{\pi}\left(\mathfrak{b}_{k, s}(u)\left[\log \left|u-(2 s-1)^{2}\right|+\frac{i \pi}{2}\right], u \in((2 s-\right.$ $\left.1)^{2},(2 s-1)^{2}+\varepsilon\right)$ are equal to their respective Taylor series in powers of $\left[u-(2 s-1)^{2}\right]^{\mathbb{Z} \geq 0}$, and all the corresponding Taylor coefficients agree.

The next lemma will provide us with special values of the determinants occurring in (3.26) and (3.27), among other things.

Lemma 3.5 (Some special determinants). Fix an integer $m \in \mathbb{Z}_{>1}$. For every $s \in \mathbb{Z} \cap\left[1,\left\lfloor\frac{m}{2}\right\rfloor+1\right]$, define thresholds $\theta_{m, s}:=\left(2 s-\frac{1+(-1)^{m}}{2}\right)^{2}$ and $\theta_{m,\lfloor m / 2\rfloor+2}=\infty$, along with Wrońskian determinants

$$
\begin{aligned}
W_{m, s}(u) & :=\operatorname{det}\left(D^{i-1} \mathcal{F}_{m, j}(u)\right)_{i \in \mathbb{Z} \cap[1, m], j \in \mathbb{Z} \cap([1,\lfloor m / 2\rfloor+1-s] \cup[\lfloor m / 2\rfloor+2, m+s])}, \\
w_{m, s}(u) & :=\operatorname{det}\left(D^{i-1} \mathcal{F}_{m, j}(u)\right)_{i \in \mathbb{Z} \cap[1, m-1], j \in \mathbb{Z} \cap([1,\lfloor m / 2\rfloor+1-s] \cup[\lfloor m / 2\rfloor+2, m-1+s]),}
\end{aligned}
$$

so that we have

$$
\begin{aligned}
& C^{\infty}\left(\theta_{m, s}, \theta_{m, s+1}\right) \cap \operatorname{ker} \widetilde{L}_{m} \\
= & \operatorname{span}_{\mathbb{C}}\left\{\mathcal{F}_{m, j}(u), u \in\left(\theta_{m, s}, \theta_{m, s+1}\right) \mid j \in \mathbb{Z} \cap\left[1,\left\lfloor\frac{m}{2}\right\rfloor+1-s\right] \cup\left[\left\lfloor\frac{m}{2}\right\rfloor+2, m+s\right]\right\}
\end{aligned}
$$

and

$$
\begin{aligned}
& W_{m, s}(u)=\frac{1+(m+1) \delta_{s,\lfloor m / 2\rfloor+1}}{(-1)^{\frac{s(s+1)}{2}}-\left(\left\lfloor\frac{m}{2}\right\rfloor+1\right) s} \frac{\Lambda_{m}}{\left|\mathcal{L}_{m}(u)\right|^{m / 2}}, \quad u \in\left(\theta_{m, s}, \theta_{m, s+1}\right), \\
& w_{m, s}\left(\theta_{m, s}\right)=\frac{1+(m+1) \delta_{s,\lfloor m / 2\rfloor+1}}{(-1)^{\frac{s(s+1)}{2}}-\left(\left\lfloor\frac{m}{2}\right\rfloor+1\right) s} \frac{2^{m+\left\lfloor\frac{m-1}{2}\right\rfloor} \theta_{m, s}^{\frac{m-1}{4}}}{(m-2) ! !} \frac{(-\sqrt{\pi})^{\frac{1+(-1)^{m}}{2}} \Lambda_{m}}{\left|\mathcal{L}_{m}^{\prime}\left(\theta_{m, s}\right)\right|^{m / 2}},
\end{aligned}
$$

where $\Lambda_{m}$ is defined in (2.37) and $\mathcal{L}_{m}(u)$ in (1.8). The expression

$$
\begin{aligned}
\mathcal{L}_{m}^{\prime}\left(\theta_{m, s}\right) & =\theta_{m, s}^{\left\lfloor\left\lfloor\frac{m+1}{2}\right\rfloor\right.} \prod_{j \in \mathbb{Z} \cap([1, s-1] \cup[s+1,\lfloor m / 2\rfloor+1])}\left[\theta_{m, s}-\left(2 j-\frac{1+(-1)^{m}}{2}\right)^{2}\right] \\
& =(-1)^{\left\lfloor\frac{m}{2}\right\rfloor+1-s} 2^{m} \theta_{m, s}^{\frac{m-1}{2}}\left(\left\lfloor\frac{m}{2}\right\rfloor+1-s\right) !\left(\left\lfloor\frac{m+1}{2}\right\rfloor+s\right) !
\end{aligned}
$$

evaluates the derivative $\mathcal{L}_{m}^{\prime}(u)=D^{1} \mathcal{L}_{m}(u)$ at $u=\theta_{m, s}$.

Proof. In view of the arguments in [49, Lemma 4.2], one can show that each off-shell Bessel moment $\mathcal{F}_{m, j}(u)$ listed in (3.50) indeed resides in the kernel space of Vanhove's operator of order $m$. What remains to be elucidated is the mechanism by which $W_{m, s-1}(u), u \in\left(\theta_{m, s-1}, \theta_{m, s}\right)$ and $W_{m, s}(u), u \in\left(\theta_{m, s}, \theta_{m, s+1}\right)$ are relayed to each other [see (3.51)], as well as the rationale behind (3.52).

Without loss of generality, we assume that $m=2 k-1$ is an odd number.

By cofactor expansion, one can compute

$$
\begin{aligned}
& \lim _{u \rightarrow(2 s)^{2}-0^{+}} \frac{W_{2 k, s-1}(u)}{\left|(2 s)^{2}-u\right|^{\frac{1}{2}-k}}=(-1)^{k-s} w_{2 k-1, s}\left((2 s)^{2}\right) \lim _{u \rightarrow(2 s)^{2}-0^{+}} \frac{D^{2 k-2} \mathcal{F}_{2 k-1, k+1-s}(u)}{\left|(2 s)^{2}-u\right|^{\frac{1}{2}-k}}, \\
& \lim _{u \rightarrow(2 s)^{2}+0^{+}} \frac{W_{2 k, s}(u)}{\left|(2 s)^{2}-u\right|^{\frac{1}{2}-k}}=w_{2 k-1, s}\left((2 s)^{2}\right) \lim _{u \rightarrow(2 s)^{2}+0^{+}} \frac{D^{2 k-2} \mathcal{F}_{2 k-1,2 k-1+s}(u)}{\left|(2 s)^{2}-u\right|^{\frac{1}{2}-k}},
\end{aligned}
$$


which involve a common factor $w_{2 k-1, s}\left((2 s)^{2}\right)$. When $s \in \mathbb{Z} \cap[1, k)$, by a procedure akin to the proof of (3.33), one can show that

$$
\begin{aligned}
& \lim _{u \rightarrow(2 s)^{2}-0^{+}}\left|(2 s)^{2}-u\right|^{k-\frac{1}{2}} D^{2 k-2} \mathcal{F}_{2 k-1, k+1-s}(u) \\
= & \frac{1}{(4 s)^{2 k-2}} \lim _{u \rightarrow(2 s)^{2}-0^{+}}\left|(2 s)^{2}-u\right|^{k-\frac{1}{2}} \mathcal{F}_{2 k-1, k+1-s}^{k}(u) \\
= & \lim _{u \rightarrow(2 s)^{2}-0^{+}} \frac{\left|(2 s)^{2}-u\right|^{k-\frac{1}{2}}}{\pi^{s}(4 s)^{2 k-2}} \int_{0}^{\infty} \frac{e^{\sqrt{u} t}}{\sqrt{2 \pi \sqrt{u} t}}\left(\frac{e^{t}}{\sqrt{2 \pi t}}\right)^{k-s}\left(\sqrt{\frac{\pi}{2 t}} e^{-t}\right)^{k+s} t^{2 k-1} \mathrm{~d} t \\
= & \frac{\Gamma\left(k-\frac{1}{2}\right)}{\sqrt{\pi}} 2^{2-3 k} s^{1-k}=(2 k-3) ! ! 2^{3-4 k} s^{1-k}
\end{aligned}
$$

and that $\lim _{u \rightarrow(2 s)^{2}+0^{+}}\left|(2 s)^{2}-u\right|^{k-\frac{1}{2}} D^{2 k-2} \mathcal{F}_{2 k-1,2 k-1+s}(u)$ evaluates to exactly the same number. This shows that the asymptotic behavior of our Wrońskian determinants match at $u \rightarrow(2 s)^{2} \pm 0^{+}$, up to a trackable sign. Therefore, we can verify (3.51) for $s \in \mathbb{Z} \cap[1, k)$ by successively applying such a matching procedure to (1.9). When $s=k$, one must exert extra care, in that

$$
\lim _{u \rightarrow(2 k)^{2}-0^{+}}\left|(2 k)^{2}-u\right|^{k-\frac{1}{2}} D^{2 k-2} \mathcal{F}_{2 k-1,1}(u)=\lim _{u \rightarrow(2 k)^{2}-0^{+}} \frac{\left|(2 k)^{2}-u\right|^{k-\frac{1}{2}}}{\pi^{k}} \frac{D^{2 k-2} \widetilde{\mathbf{I}} \mathbf{K M}(1,2 k ; 1 \mid u)}{2 k+1}
$$

carries a factor of $\frac{1}{2 k+1}$.

As a by-product, the common factor $w_{2 k-1, s}\left((2 s)^{2}\right)$ during this matching procedure can be identified with the right-hand side of (3.52).

3.3. Quadratic relations for off-shell Bessel moments. With the preparations in the last two lemmata, we can compute $\mathbf{S}_{m}$ for all $m \in \mathbb{Z}_{>0}$.

Proposition 3.6 (Explicit formulae for $\mathbf{S}_{m}$ ). (a) If $a \in \mathbb{Z} \cap[1, k]$, then

$$
\begin{aligned}
& \frac{(a-1) !(2 k+1-a) !\left(\mathbf{S}_{2 k-1}\right)_{a, b}}{2^{2 k-1}(-1)^{\left\lfloor\frac{a}{2}\right\rfloor-k}\left(1+2 k \delta_{a, 1}\right)} \\
& = \begin{cases}-\frac{1+(-1)^{a+1}}{2}\left(\begin{array}{c}
2 k-a \\
k
\end{array}\right)(2 k+1), & b=1, \\
\frac{1+(-1)^{a+b} k-a+1}{\left.2(-1)^{\frac{b}{2}}\right]} \sum_{s=1}(-1)^{s}\left(\begin{array}{c}
2 k+1-a \\
k+s
\end{array}\right)\left(\begin{array}{c}
k-s \\
b-1
\end{array}\right), & b \in \mathbb{Z} \cap[2, k], \\
\frac{(-1)^{a-1}+(-1)^{b-k}}{2(-1)^{\frac{b-k}{2}}} \sum_{s=1}^{k-a+1}(-1)^{s}\left(\begin{array}{c}
2 k+1-a \\
k+s
\end{array}\right)\left[\left(\begin{array}{c}
k-s \\
b-k+1
\end{array}\right)+(-1)^{b-k}\left(\begin{array}{c}
k+s \\
b-k+1
\end{array}\right)\right], & b \in \mathbb{Z} \cap[k+1,2 k-1],\end{cases}
\end{aligned}
$$

where the first row can be rewritten as

$$
\frac{(k !)^{2}\left(\mathbf{S}_{2 k-1}\right)_{1, b}}{(-4)^{k-1}(2 k+1)}= \begin{cases}2 k+1, & b=1, \\
\frac{1+(-1)^{b+1}}{\left.(-1)^{\frac{b}{2}}\right]} \frac{b}{2 k+1-b}\left(\begin{array}{l}
k \\
b
\end{array}\right), & b \in \mathbb{Z} \cap[2, k], \\
\frac{1+(-1)^{b-k}}{\left.(-1) \frac{b-k}{2}\right]}\left(\begin{array}{c}
k \\
b-k+1
\end{array}\right), & b \in \mathbb{Z} \cap[k+1,2 k-1],\end{cases}
$$

which entails

$$
\left(\mathbf{S}_{2 k-1}\right)_{a, b}=\frac{(-4)^{k-1}}{(k !)^{2}} \frac{\left[1+(-1)^{a-k}\right]\left[1+(-1)^{b-k}\right]}{(-1)^{\left\lfloor\frac{a-k}{2}\right\rfloor+\left\lfloor\frac{b-k}{2}\right\rfloor}}\left(\begin{array}{c}
k \\
a-k+1
\end{array}\right)\left(\begin{array}{c}
k \\
b-k+1
\end{array}\right)
$$

for $a, b \in \mathbb{Z} \cap[k+1,2 k-1]$. 
(b) If $a \in \mathbb{Z} \cap[1, k]$, then

$$
\begin{aligned}
& \frac{(a-1) !(2 k+2-a) !\left(\mathbf{S}_{2 k}\right)_{a, b}}{2^{2 k-1}(-1)^{k}\left[1+(2 k+1) \delta_{a, 1}\right]} \\
& = \begin{cases}\frac{1+(-1)^{a+b+1}}{(-1)^{\left.\frac{a}{2}\right\rfloor+\left[\frac{b-1}{2}\right]-1}}\left[1+(2 k+1) \delta_{b, 1}\right] \sum_{s=1}^{k+2-a}(-1)^{s}\left(\begin{array}{c}
2 k+2-a \\
k+s
\end{array}\right)\left(\begin{array}{c}
k+1-s \\
b-1
\end{array}\right), & b \in \mathbb{Z} \cap[1, k+1], \\
\frac{1+(-1)^{a+b-k-1}}{(-1)^{\left[\frac{a-1}{2}\right]+\left[\frac{b-k}{2}\right]}} \sum_{s=1}^{k+2-a}(-1)^{s}\left(\begin{array}{c}
2 k+2-a \\
k+s
\end{array}\right)\left[\left(\begin{array}{c}
k+1-s \\
b-k
\end{array}\right)+(-1)^{b-k-1}\left(\begin{array}{c}
k+s \\
b-k
\end{array}\right)\right], & b \in \mathbb{Z} \cap[k+2,2 k],\end{cases}
\end{aligned}
$$

where the first row can be rewritten as

$$
\left(\mathbf{S}_{2 k}\right)_{1, b}= \begin{cases}\frac{2^{2 k} b}{(k !)^{2}} \frac{1+(-1)^{b}}{(-1)^{\frac{b-1}{2}} \frac{\left(\begin{array}{c}
k+1 \\
b
\end{array}\right)}{2 k+2-b},} & b \in \mathbb{Z} \cap[1, k+1] \\
0, & b \in \mathbb{Z} \cap[k+2,2 k] .\end{cases}
$$

If $a, b \in \mathbb{Z} \cap[k+2,2 k]$, then $\left(\mathbf{S}_{2 k}\right)_{a, b}=0$.

Proof. In the proof below, we will repeatedly use the Wrońskian determinant $w_{m, s}(u)$ introduced in 3.49.

(a) The inputs from Lemma 3.4 (a) and Lemma $3.5(m=2 k-1)$ allow us to set up the following identities:

$$
\begin{aligned}
& \frac{4^{2-3 k}\left(-s^{2}\right)^{1-k}}{(-1)^{k s-\frac{s(s+1)}{2}}} \frac{\left|\mathcal{L}_{2 k-1}^{\prime}\left((2 s)^{2}\right)\right|}{1+2 k \delta_{s, k}} \frac{\left|\mathcal{L}_{2 k-1}(u)\right|^{k-\frac{3}{2}}}{\Lambda_{2 k-1}} w_{2 k-1, s}(u) \\
= & \begin{cases}(-1)^{\left\lfloor\frac{k-s}{2}\right\rfloor+(k-1)(k-s)} \operatorname{ReF}_{k, s}^{1}(u), & (-1)^{k-s}=+1, \\
(-1)^{\left\lfloor\frac{k-s-1}{2}\right\rfloor+(k-1)(k-s-1)} \operatorname{ImF}_{k, s}^{1}(u), & (-1)^{k-s}=-1,\end{cases}
\end{aligned}
$$

when $u \in\left((2 s-2)^{2},(2 s)^{2}\right)$, and

$$
\begin{aligned}
& \frac{4^{2-3 k}\left(-s^{2}\right)^{1-k}}{(-1)^{k s-\frac{s(s+1)}{2}}} \frac{\left|\mathcal{L}_{2 k-1}^{\prime}\left((2 s)^{2}\right)\right|}{1+2 k \delta_{s, k}} \frac{\left|\mathcal{L}_{2 k-1}(u)\right|^{k-\frac{3}{2}}}{\Lambda_{2 k-1}} w_{2 k-1, s}(u) \\
= & \begin{cases}(-1)^{\left\lfloor\frac{k-s}{2}\right\rfloor+(k-1)(k-s)} \operatorname{ReF}_{k, s}^{1}(u), & (-1)^{k-s}=-1, \\
(-1)^{\left\lfloor\frac{k-s-1}{2}\right\rfloor+(k-1)(k-s-1)} \operatorname{ImF}_{k, s}^{1}(u), & (-1)^{k-s}=+1,\end{cases}
\end{aligned}
$$

when $u \in\left((2 s)^{2},(2 s+2)^{2}\right)$. Here, the left-hand sides of (3.62) and (3.63) behave like a constant multiple of $1+\left[u-(2 s)^{2}\right] f_{k, s}(u)$ as $u \rightarrow(2 s)^{2}$, where $f_{k, s}(u)$ is the real-analytic function mentioned in Lemma 3.4(a), Thus, one can establish equalities in (3.62) and (3.63) after comparing leading order asymptotic behavior.

Without loss of generality, we momentarily consider the cases where $k$ is odd. We begin with the $k$ th row of $\mathbf{S}_{2 k-1}$ [cf. (2.36)], which concerns

$$
W\left[\mathcal{F}_{2 k-1,1}(u), \ldots, \overline{\mathcal{F}_{2 k-1, k}(u)}, \ldots, \mathcal{F}_{2 k-1,2 k-1}(u)\right]=w_{2 k-1,1}(u) .
$$

Setting $s=1$ in (3.62), while simplifying $\left|\mathcal{L}_{2 k-1}^{\prime}(4)\right|=2^{4 k-3}(k+1)(k-1) ! k$ ! and $\left.(-1)^{\left\lfloor\frac{k-s}{2}\right\rfloor+(k-1)(k-s)+(k-1)+k s-\frac{s(s+1)}{2}}\right|_{s=1}=(-1)^{\left\lfloor\frac{k-1}{2}\right\rfloor+k-1}$, we may spell out

$$
\begin{aligned}
& \frac{\left|\mathcal{L}_{2 k-1}(u)\right|^{k-\frac{3}{2}}}{\Lambda_{2 k-1}}(-1)^{k} w_{2 k-1,1}(u)=\frac{2^{2 k-1}(-1)^{\left\lfloor\frac{k-1}{2}\right\rfloor-1}}{(k-1) !(k+1) !} \operatorname{Re}_{k, 1}^{1}(u) \\
= & \frac{2^{2 k-1}(-1)^{\left\lfloor\frac{k-1}{2}\right\rfloor-1}}{(k-1) !(k+1) !}\left\{(2 k+1) \mathcal{F}_{2 k-1,1}^{\ell}(u)+\sum_{j=1}^{\left\lfloor\frac{k-1}{2}\right\rfloor}(-1)^{j}\left(\begin{array}{c}
k-1 \\
2 j
\end{array}\right) \mathcal{F}_{2 k-1,2 j+1}^{\ell}(u)\right.
\end{aligned}
$$




$$
\left.+\sum_{j=1}^{\left\lfloor\frac{k}{2}\right\rfloor}(-1)^{j}\left[\left(\begin{array}{c}
k-1 \\
2 j+1
\end{array}\right)+\left(\begin{array}{c}
k+1 \\
2 j+1
\end{array}\right)\right] \mathcal{F}_{2 k-1,2 j+k}^{\ell}(u)\right\}, \quad u \in(0,16),
$$

which explains why

$$
\frac{(k-1) !(k+1) !\left(\mathbf{S}_{2 k-1}\right)_{k, b}}{2^{2 k-1}(-1)\left\lfloor\frac{k-1}{2}\right\rfloor-1}= \begin{cases}2 k+1, & b=1, \\
\frac{1-(-1)^{b}}{\left.2(-1) \frac{b}{2}\right\rfloor}\left(\begin{array}{c}
k-1 \\
b-1
\end{array}\right), & b \in \mathbb{Z} \cap[2, k], \\
\frac{1-(-1)^{b}}{2(-1) ! \frac{b-k}{2}}\left[\left(\begin{array}{c}
k-1 \\
b-k+1
\end{array}\right)+\left(\begin{array}{c}
k+1 \\
b-k+1
\end{array}\right)\right], & b \in \mathbb{Z} \cap[k+1,2 k-1],\end{cases}
$$

when $k$ is odd. Then we proceed to the $(k-1)$-st row. We observe that

$$
\begin{aligned}
& \frac{(-1)^{k-1} W\left[\mathcal{F}_{2 k-1,1}(u), \ldots, \overline{\mathcal{F}_{2 k-1, k-1}(u)}, \ldots, \mathcal{F}_{2 k-1,2 k-1}(u)\right]}{\left|\mathcal{L}_{2 k-1}(u)\right|^{\frac{3}{2}-k} \Lambda_{2 k-1}} \\
= & \frac{W\left[\mathcal{F}_{2 k-1,1}(u), \ldots, \mathcal{F}_{2 k-1, k-2}(u), \mathcal{F}_{2 k-1, k}(u), \ldots, \mathcal{F}_{2 k-1,2 k-1}(u)\right]}{\left|\mathcal{L}_{2 k-1}(u)\right|^{\frac{3}{2}-k} \Lambda_{2 k-1}} \\
= & \frac{W\left[\mathcal{F}_{2 k-1,1}(u), \ldots, \mathcal{F}_{2 k-1, k-2}(u), \operatorname{Re} F_{k, 1}^{1}(u), \mathcal{F}_{2 k-1, k+1}(u), \ldots, \mathcal{F}_{2 k-1,2 k-1}(u)\right]}{\left.(-1)^{\left\lfloor\frac{k-1}{2}\right.}\right\rfloor\left|\mathcal{L}_{2 k-1}(u)\right|^{\frac{3}{2}-k} \Lambda_{2 k-1}}
\end{aligned}
$$

can be developed into a Taylor series centered at $u_{0}=4$ for $u \in(4-\varepsilon, 4)$, by virtue of (3.28). Such a function admits real-analytic continuation to

$$
-\frac{W\left[\mathcal{F}_{2 k-1,1}(u), \ldots, \mathcal{F}_{2 k-1, k-2}(u), \operatorname{ImF}_{k, 1}^{1}(u), \mathcal{F}_{2 k-1, k+1}(u), \ldots, \mathcal{F}_{2 k-1,2 k-1}(u)\right]}{(-1)^{\left\lfloor\frac{k-1}{2}\right\rfloor}\left|\mathcal{L}_{2 k-1}(u)\right|^{\frac{3}{2}-k} \Lambda_{2 k-1}}
$$

for $u \in(4,16)$, by a comparison between (3.28) and (3.29). Since (2.38) and (2.39) tell us that

$$
\begin{aligned}
& \operatorname{ImF}_{k, 1}^{1}(u)-\left\{(-1)^{\left\lfloor\frac{k}{2}\right\rfloor-1}(k-1) \mathcal{F}_{2 k-1, k-1}(u)+(-1)^{\left\lfloor\frac{k+1}{2}\right\rfloor} \mathcal{F}_{2 k-1,2 k}(u)\right\} \\
\in & \operatorname{span}_{\mathbb{R}}\left\{\mathcal{F}_{2 k-1, j}(u), u \in(4,16) \mid j \in \mathbb{Z} \cap([1, k-2] \cup[k+1,2 k-1])\right\},
\end{aligned}
$$

we can convert (3.68) into [cf. (3.62) and (3.63)] a $\mathbb{Q}$-linear combination of $\left|\mathcal{L}_{2 k-1}(u)\right|^{k-\frac{3}{2}} w_{2 k-1,1}(u)$ and $\left|\mathcal{L}_{2 k-1}(u)\right|^{k-\frac{3}{2}} w_{2 k-1,2}(u)$, in the following manner:

$$
\left.\begin{array}{rl} 
& \frac{\left|\mathcal{L}_{2 k-1}(u)\right|^{k-\frac{3}{2}}}{\Lambda_{2 k-1}}\left\{W\left[\mathcal{F}_{2 k-1,1}(u), \ldots, \mathcal{F}_{2 k-1, k-2}(u),(k-1) \mathcal{F}_{2 k-1, k-1}(u), \mathcal{F}_{2 k-1, k+1}(u), \ldots, \mathcal{F}_{2 k-1,2 k-1}(u)\right]\right. \\
& \left.+W\left[\mathcal{F}_{2 k-1,1}(u), \ldots, \mathcal{F}_{2 k-1, k-2}(u), \mathcal{F}_{2 k-1,2 k}(u), \mathcal{F}_{2 k-1, k+1}(u), \ldots, \mathcal{F}_{2 k-1,2 k-1}(u)\right]\right\} \\
= & \frac{2^{2 k-1}(-1)^{\left\lfloor\frac{k-1}{2}\right\rfloor-1}}{(k-1) !(k+1) !}\left[(k-1) \operatorname{Im}_{k, 1}^{1}(u)-\frac{k-1}{k+2} \operatorname{Im}_{k, 2}^{1}(u)\right] \\
= & \frac{2^{2 k-1}(-1)^{\left\lfloor\frac{k-1}{2}\right\rfloor-1}}{(k-2) !(k+2) !}\left\{\sum_{j=0}^{\left\lfloor\frac{k}{2}\right\rfloor-1}(-1)^{j}\left[(k+2)\left(\begin{array}{c}
k-1 \\
2 j+1
\end{array}\right)-\left(\begin{array}{c}
k-2 \\
2 j+1
\end{array}\right)\right] \mathcal{F}_{2 k-1,2 j+2}^{\ell}(u)\right. \\
& -\sum_{j=1}^{\left\lfloor\frac{k-1}{2}\right\rfloor}(-1)^{j}\left[(k+2)\left(\begin{array}{c}
k-1 \\
2 j
\end{array}\right)-(k+2)\left(\begin{array}{c}
k+1 \\
2 j
\end{array}\right)-\left(\begin{array}{c}
k-2 \\
2 j
\end{array}\right)+\left(\begin{array}{c}
k+2 \\
2 j
\end{array}\right)\right] \mathcal{F}_{2 k-1,2 j+k-1}^{\ell}(u)
\end{array}\right\} .
$$

On the right-hand side of this expression, the last summands of $\operatorname{ImF}_{k, 1}^{1}(u)$ and $\frac{1}{k+2} \operatorname{ImF}_{k, 2}^{1}(u)[$ which are $(-1)^{\frac{k+1}{2}}\left(\begin{array}{l}k+1 \\ k+1\end{array}\right) \mathcal{F}_{2 k-1,2 k}^{\ell}(u)$ and $(-1)^{\frac{k+1}{2}} \frac{1}{k+2}\left(\begin{array}{l}k+2 \\ k+1\end{array}\right) \mathcal{F}_{2 k-1,2 k}^{\ell}(u)$, respectively] cancel each other. Thanks to this cancelation of terms undefined for $u \in(0,4)$, the right-hand side of (3.70) real-analytically continues to $u \in(0,16)$, and is compatible with our statement about $\left(\mathbf{S}_{2 k-1}\right)_{k-1, b}$ in (3.57). 
Proceeding as the last paragraph, we obtain the following real-analytic continuations of (3.62):11

$$
\begin{aligned}
& \frac{(a-1) !(2 k+1-a) !}{2^{2 k-1}\left(1+2 k \delta_{a, 1}\right)} \frac{\left|\mathcal{L}_{2 k-1}(u)\right|^{k-\frac{3}{2}}}{\Lambda_{2 k-1}} \frac{\operatorname{det}\left(D^{i-1} \mathcal{F}_{2 k-1, j}(u)\right)_{i \in \mathbb{Z} \cap[1,2 k-2], j \in \mathbb{Z} \cap([1, a-1] \cup[a+1, k-t] \cup[k+1,2 k-1+t])}}{(-1)^{k t-\frac{t(t-1)}{2}}(-1)^{\left\lfloor\frac{a}{2}\right\rfloor-k}(-1)^{a+1}} \\
= & \sum_{b=1}^{k-t}\left[\left(1+2 k \delta_{b, 1}\right) \frac{1+(-1)^{a+b}}{2(-1)^{\left\lfloor\frac{b}{2}\right\rfloor}} \sum_{s=t+1}^{k+1-a}(-1)^{s}\left(\begin{array}{c}
2 k+1-a \\
k+s
\end{array}\right)\left(\begin{array}{c}
k-s \\
b-1
\end{array}\right)\right] \mathcal{F}_{2 k-1, b}(u) \\
& +\sum_{b=k+1}^{2 k-1+t}\left\{\frac{(-1)^{a-1}+(-1)^{b+k}}{2(-1)^{\left\lfloor\frac{b-k}{2}\right\rfloor}} \sum_{s=t+1}^{k+1-a}(-1)^{s}\left(\begin{array}{c}
2 k+1-a \\
k+s
\end{array}\right) \times\right. \\
& \left.\times\left[\left(\begin{array}{c}
k-s \\
b-k+1
\end{array}\right)-(-1)^{b-k+1}\left(\begin{array}{c}
k+s \\
b-k+1
\end{array}\right)\right]\right\} \mathcal{F}_{2 k-1, b}(u),
\end{aligned}
$$

where $t \in \mathbb{Z} \cap[0, k-1], a=k-1-t, u \in\left((2 t)^{2},(2 t+2)^{2}\right)$. [If we set $t \in \mathbb{Z} \cap[0, k-1], a=k-t, u \in$ $\left((2 t)^{2},(2 t+2)^{2}\right)$ in 3.62 , we recover the original (3.62).] Recursively performing real-analytic continuations with the aid of (3.63), down-stepping $\sqrt{u}$ by an amount of 2 every time, we can further extend the validity of (3.62*) to $t \in \mathbb{Z} \cap[0, k-1], a \in \mathbb{Z} \cap[1, k-t], u \in\left((2 t)^{2},(2 t+2)^{2}\right)$. In particular, setting $t=0$ in (3.62 $)$, one can verify the expression $\left(\mathbf{S}_{2 k-1}\right)_{a, b}$ in (3.57), for each $a \in \mathbb{Z} \cap[1, k]$.

One can readily generalize the procedures in the last two paragraphs [which essentially revolve around real-analytic continuations of $w_{2 k-1, s}(u)$ ] to even numbers $k$, thus establishing (3.57) in its entirety.

Plugging two combinatorial identities

$$
\begin{aligned}
& \sum_{s=1}^{k}(-1)^{s}\left(\begin{array}{c}
2 k \\
k+s
\end{array}\right)\left(\begin{array}{c}
k-s \\
n
\end{array}\right)=-\frac{2 k}{2 k-n}\left(\begin{array}{c}
2 k-1 \\
k
\end{array}\right)\left(\begin{array}{c}
k-1 \\
n
\end{array}\right), \\
& \sum_{s=1}^{k}(-1)^{s}\left(\begin{array}{c}
2 k \\
k+s
\end{array}\right)\left(\begin{array}{c}
k+s \\
n
\end{array}\right)=-\frac{2 k}{2 k-n}\left(\begin{array}{c}
2 k-1 \\
k
\end{array}\right)\left(\begin{array}{l}
k \\
n
\end{array}\right)
\end{aligned}
$$

into (3.57) for the expression $\left(\mathbf{S}_{2 k-1}\right)_{1, b}$, we obtain (3.58).

Let $k \in \mathbb{Z}_{\geq 2}$ and $r \in \mathbb{Z} \cap[k+1,2 k-1]$. Through elementary manipulations of rows and columns in a determinant (bearing in mind that $\left|\operatorname{det} \boldsymbol{\beta}_{2 k-2}(u)\right|=2^{(k-1)(2 k-3)}|u|^{(k-1)^{2}}$ ), one can identify

$$
\begin{aligned}
& \left|\mathcal{L}_{2 k-1}(u)\right|^{k-\frac{3}{2}}\left\{(-1)^{r+1} W\left[\mathcal{F}_{2 k-1,1}(u), \ldots, \overline{\mathcal{F}_{2 k-1, r}(u)}, \ldots, \mathcal{F}_{2 k-1,2 k-1}(u)\right]\right. \\
& \left.-\frac{1+(-1)^{r-k}}{(-1)^{\left.\frac{r-k}{2}\right\rfloor}}\left(\begin{array}{c}
k \\
r-k+1
\end{array}\right) W\left[\overline{\mathcal{F}_{2 k-1,1}(u)}, \ldots, \mathcal{F}_{2 k-1, r}(u), \ldots, \mathcal{F}_{2 k-1,2 k-1}(u)\right]\right\}
\end{aligned}
$$

for $u \in(0,4)$ with a constant multiple of

$$
\left(\prod_{j=1}^{k}\left[(2 j)^{2}-u\right]\right)^{k-\frac{3}{2}} \operatorname{det}\left(\begin{array}{rr}
\boldsymbol{\iota}_{k-1}(u) & \boldsymbol{\iota}_{k-1}(u) \\
\boldsymbol{\kappa}_{k-1, r}(u) & \dot{\boldsymbol{\kappa}}_{k-1, r}(u)
\end{array}\right),
$$

where the blocks are designed as follows:

(1) We have $\boldsymbol{\iota}_{k-1}(u)=\left(\mathcal{F}_{2 k-1, a+1}^{b}(u)\right)_{1 \leq a, b \leq k-1}$ and $\boldsymbol{\iota}_{k-1}(u)=\left(\sqrt{u} \mathcal{F}_{2 k-1, a+1}^{b}(u)\right)_{1 \leq a, b \leq k-1}$;

(2) The first row of $\boldsymbol{\kappa}_{k-1, r}(u)\left[\operatorname{resp} . \dot{\boldsymbol{\kappa}}_{k-1, r}(u)\right]$ is given by $\left(\boldsymbol{\kappa}_{k-1, r}(u)\right)_{1, b}=\pi \mathcal{F}_{2 k-1,1}^{b}(u)+\frac{2 k}{2 k+1} \mathcal{F}_{2 k-1,2}^{b}(u) \log \frac{\sqrt{u}}{2}$ $\left[\operatorname{resp} .\left(\boldsymbol{\kappa}_{k-1, r}(u)\right)_{1, b}=\sqrt{u}\left[\pi \dot{\mathcal{F}}_{2 k-1,1}^{b}(u)+\frac{2 k}{2 k+1} \dot{\mathcal{F}}_{2 k-1,2}^{b}(u) \log \frac{\sqrt{u}}{2}\right]\right]$;

\footnotetext{
${ }^{11}$ Similar to Proposition 3.2, here we require that all the column indices be arranged in increasing order (even when they are not consecutive integers).
} 
(3) The next $k-3$ rows in $\boldsymbol{\kappa}_{k-1, r}(u)\left[\operatorname{resp} . \boldsymbol{\kappa}_{k-1, r}(u)\right]$ form a subset of $\left\{\left(\pi \mathcal{F}_{2 k-1, a}^{b}(u)+\mathcal{F}_{2 k-1, a-k+2}^{b}(u)\right.\right.$ $\left.\left.\log \frac{\sqrt{u}}{2}\right)_{1 \leq b \leq k-1} \mid a \in \mathbb{Z} \cap[k+1,2 k-2]\right\}\left[\operatorname{resp} .\left\{\left(\sqrt{u}\left[\pi \dot{\mathcal{F}}_{2 k-1, a}^{b}(u)+\dot{\mathcal{F}}_{2 k-1, a-k+2}^{b}(u) \log \frac{\sqrt{u}}{2}\right]\right)_{1 \leq b \leq k-1} \mid a \in\right.\right.$ $\mathbb{Z} \cap[k+1,2 k-2]\}]$

(4) The last row of $\boldsymbol{\kappa}_{k-1, r}(u)$ is specified by [cf. (2.51) and (2.52)]

$$
\left(\boldsymbol{\kappa}_{k-1, r}(u)\right)_{k-1, b}= \begin{cases}u^{(k-2) / 2} O_{k}^{b}(u), & k \in 1+2 \mathbb{Z}_{>0}, \\ u^{(k-2) / 2} E_{k}^{b}(u), & k \in 2 \mathbb{Z}_{>0}, r \neq 2 k-1, \\ u^{(k-2) / 2}\left[\pi \mathcal{F}_{2 k-1,2 k-2}^{b}(u)+\mathcal{F}_{2 k-1, k}^{b}(u) \log \frac{\sqrt{u}}{2}\right], & k \in 2 \mathbb{Z}_{>0}, r=2 k-1,\end{cases}
$$

while the last row of $\boldsymbol{\kappa}_{k-1, r}(u)$ is specified by [cf. (2.51] and 2.52]]

$$
\left(\dot{\boldsymbol{\kappa}}_{k-1, r}(u)\right)_{k-1, b}= \begin{cases}u^{(k-1) / 2} \hat{O}_{k}^{b}(u), & k \in 1+2 \mathbb{Z}_{>0}, \\ u^{(k-1) / 2} \dot{E}_{k}^{b}(u), & k \in 2 \mathbb{Z}_{>0}, r \neq 2 k-1, \\ u^{(k-1) / 2}\left[\pi \dot{\mathcal{F}}_{2 k-1,2 k-2}^{b}(u)+\dot{\mathcal{F}}_{2 k-1, k}^{b}(u) \log \frac{\sqrt{u}}{2}\right], & k \in 2 \mathbb{Z}_{>0}, r=2 k-1 .\end{cases}
$$

[For $k=2$, the instructions in items (3) and (4) must be ignored, whereupon we are reduced to the case already treated in Corollary 2.11]. According to the arguments in Proposition 2.10(a), the expression in (3.74) defines a holomorphic function in a non-void $\mathbb{C}$-neighborhood of $u=0$, hence a member of $\operatorname{span}_{\mathbb{C}}\left\{\mathcal{F}_{2 k-1, a}(u) \mid a \in \mathbb{Z} \cap[2, k]\right\}$. This completes the proof of (3.59).

(b) By (3.31), the expression

$$
\left[u-(2 s-1)^{2}\right]^{k-1}\left\{D^{n} \mathcal{I}_{2 k, s}^{1}(u)+\frac{(-1)^{k-s-1}-i}{\pi(1-i)}\left[D^{n} \mathfrak{5}_{k, s}(u)\right]\left[\log \left|u-(2 s-1)^{2}\right|-\frac{i \pi}{2}\right]-D^{n} \mathfrak{L}_{k, s}(u)\right\}
$$

for $n \in \mathbb{Z} \cap[0,2 k-1]$ is equal to a convergent Taylor series in powers of $\left[u-(2 s-1)^{2}\right]^{\mathbb{Z}_{\geq 0}}$ for $u \in$ $\left((2 s-1)^{2}-\varepsilon,(2 s-1)^{2}\right)$, where $\varepsilon>0$. Accordingly, for each $s \in \mathbb{Z} \cap[1, k]$, there exists a non-vanishing constant $c_{k, s}$ that allows us to identify the following formula

$$
\begin{aligned}
& {\left[\mathcal{L}_{2 k}(u)\right]^{k-1}\left\{W \left[\mathcal{F}_{2 k, 1}(u), \ldots, \mathcal{F}_{2 k, k-s}(u), \mathcal{F}_{2 k, k+2-s}(u),\right.\right.} \\
& \left.\left.\mathcal{F}_{2 k, k+2}(u), \ldots, \mathcal{F}_{2 k, 2 k-1+s}(u)\right]+c_{k, s} w_{2 k, s}(u) \log \left|u-(2 s-1)^{2}\right|\right\}
\end{aligned}
$$

with another convergent Taylor series in powers of $\left[u-(2 s-1)^{2}\right]^{\mathbb{Z} \geq 0}$ for $u \in\left((2 s-1)^{2}-\varepsilon,(2 s-1)^{2}\right)$. In the light of this, the function $\left[\mathcal{L}_{2 k}(u)\right]^{k-1} w_{2 k, s}(u)$ must be a constant multiple of $\tilde{5}_{k, s}(u)$, and the constant in question can be determined through Lemma $3.5(m=2 k)$.

In fact, the arguments in the last paragraph lead us to

$$
\frac{\left|\mathcal{L}_{2 k}(u)\right|^{k-1}}{\Lambda_{2 k}} w_{2 k, s}(u)=-\frac{2^{4 k}(2 s-1)^{2 k-1}}{(-1)^{\left.\frac{k-s}{2}\right\rfloor+\frac{(2 k-s)(s+1)}{2}}} \frac{\left(\mathfrak{G}_{k, s}(u)\right.}{\left|\mathcal{L}_{k}^{\prime}\left((2 s-1)^{2}\right)\right|}
$$

for $u \in\left((\max \{0,2 s-3\})^{2},(2 s+1)^{2}\right), s \in \mathbb{Z} \cap[1, k]$. Setting $s=1$ in the formula above, we recover the expression for $\left(\mathbf{S}_{2 k}\right)_{k+1, b}, b \in \mathbb{Z} \cap[1,2 k]$, as claimed in (3.60). Setting $s=2$ in the last displayed formula, and performing real-analytic continuation of $\mathfrak{L}_{k, 1}(u)$ [which allows us to trade $\mathcal{F}_{2 k, 2 k+1}(u), u \in(1,9)$ for $\mathcal{F}_{2 k, k+1}(u), u \in(0,1)$, up to some trailing terms contributed by $\left.\mathfrak{b}_{k, 1}(u)\right]$, we can verify the formula for $\left(\mathbf{S}_{2 k}\right)_{k, b}, b \in \mathbb{Z} \cap[1,2 k]$, as claimed in (3.60). Subsequently, after finitely many steps of real-analytic continuations, we can check the combinatorial expression for $\left(\mathbf{S}_{2 k}\right)_{a, b}, a \in \mathbb{Z} \cap[2, k], b \in \mathbb{Z} \cap[1,2 k]$.

We can directly compute $\left(\mathbf{S}_{2 k}\right)_{1, b}, b \in \mathbb{Z} \cap[2, k]$ from our knowledge of $-\left(\mathbf{S}_{2 k}\right)_{b, 1}, b \in \mathbb{Z} \cap[2, k]$.

We can deduce $\left(\mathbf{S}_{2 k}\right)_{a, b}=0$ for $a, b \in \mathbb{Z} \cap(\{1\} \cup[k+2,2 k])$ from Proposition 2.10(b).

At this point, we have verified all the statements in Theorem 1.1, effectively proving the following matrix inversion for $u \in(0,1)$ :

$$
\left[\mathbf{W}_{m}(u)\right]^{-1}=\left|\mathcal{L}_{m}(u)\right| \mathbf{S}_{m} \mathbf{W}_{m}^{\mathrm{T}}(u) \mathbf{V}_{m}(u),
$$


which in turn, can be recast into the following alternative formulation of quadratic relations for off-shell Bessel moments:

$$
\mathbf{W}_{m}^{\mathrm{T}}(u) \mathbf{V}_{m}(u) \mathbf{W}_{m}(u)=\frac{\mathbf{S}_{m}^{-1}}{\left|\mathcal{L}_{m}(u)\right|}
$$

To prepare for an inductive computation of $\mathbf{S}_{m}^{-1}$ in Proposition 4.2, we wrap up this section with a

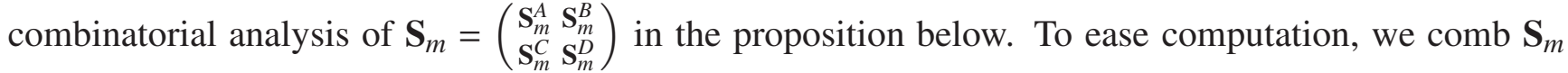
with a reshuffling-rescaling matrix $\boldsymbol{\xi}_{m} \in \mathbb{Q}^{m \times m}$ whose elements are

$$
\left(\boldsymbol{\xi}_{m}\right)_{a, b}:= \begin{cases}\delta_{a, b-1}, & a \in \mathbb{Z} \cap\left[1,\left\lfloor\frac{m}{2}\right\rfloor\right], \\ \frac{m+2}{m+1} \delta_{1, b}, & a=\left\lfloor\frac{m}{2}\right\rfloor+1, \\ \delta_{a, b}, & a \in \mathbb{Z} \cap\left[\left\lfloor\frac{m}{2}\right\rfloor+2, m\right] .\end{cases}
$$

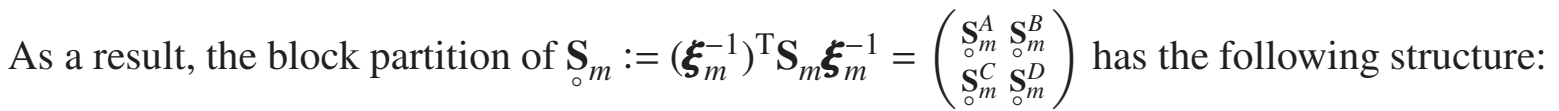

$$
\begin{cases}\left(\mathbf{S}_{\circ}^{A}\right)_{a, b}=\left(\mathbf{S}_{m}^{A}\right)_{a+1, b+1}, & a, b \in \mathbb{Z} \cap\left[1,\left\lfloor\frac{m}{2}\right\rfloor\right], \\ \left(\mathbf{S}_{m}^{B}\right)_{a, b}=\left(\mathbf{S}_{m}^{B}\right)_{a+1, b-1}, & a \in \mathbb{Z} \cap\left[1,\left\lfloor\frac{m}{2}\right\rfloor\right], b \in \mathbb{Z} \cap\left[1,\left\lfloor\frac{m+1}{2}\right\rfloor\right], \\ \left(\mathbf{S}_{o}^{D}\right)_{a, b}=\left(\mathbf{S}_{m}^{D}\right)_{a-1, b-1}, & a, b \in \mathbb{Z} \cap\left[1,\left\lfloor\frac{m+1}{2}\right\rfloor\right],\end{cases}
$$

with parity $\left(\mathbf{S}_{\circ}^{B}\right)_{a, b}=(-1)^{m+1}\left(\mathbf{S}_{\circ}^{C}\right)_{b, a}$, where extensions of (1.13)-(1.15) lead us to

$$
\begin{aligned}
& \left(\mathbf{S}_{m}^{A}\right)_{a, b}=\frac{1+(-1)^{a+b+m+1}}{2^{1-m} a !(m+1-a) !} \frac{\sum_{s=1}^{\left\lfloor\frac{m}{2}\right\rfloor+1-a}(-1)^{s}\left(\begin{array}{c}
m+1-a \\
\left\lfloor\frac{m+1}{2}\right\rfloor+s
\end{array}\right)\left(\begin{array}{c}
\left\lfloor\frac{m}{2}\right\rfloor+1-s \\
b
\end{array}\right)}{(-1)^{\left\lfloor\frac{a+1}{2}\right\rfloor+\left\lfloor\frac{b+1}{2}-\frac{1+(-1)^{m}}{4}\right\rfloor-\left\lfloor\frac{m}{2}\right\rfloor-1}}, \\
& \left(\mathbf{S}_{\circ}^{B}\right)_{a, b}=(-1)^{m+1}\left(\mathbf{S}_{o}^{C}\right)_{b, a}=\frac{1+(-1)^{a+b+m}}{2^{1-m} a !(m+1-a) !} \frac{\sum_{s=1}^{\left\lfloor\frac{m}{2}\right\rfloor+1-a}(-1)^{s}\left(\begin{array}{c}
m+1-a \\
\left\lfloor\frac{m+1}{2}\right\rfloor+s
\end{array}\right)\left[\left(\begin{array}{c}
\left\lfloor\frac{m}{2}\right\rfloor+1-s \\
b
\end{array}\right)-(-1)^{b}\left(\begin{array}{c}
\left.\frac{m+1}{2}\right\rfloor+s \\
b
\end{array}\right)\right]}{(-1)^{a m+\left\lfloor\frac{a+1}{2}-\frac{1+(-1)^{m}}{4}\right\rfloor+\left\lfloor\frac{b-1}{2}+\frac{1+(-1)^{m}}{4}\right\rfloor-\left\lfloor\frac{m+1}{2}\right\rfloor}},
\end{aligned}
$$

1.14

and

$$
\left(\mathbf{S}_{\circ}^{D}\right)_{a, b}=\frac{1+(-1)^{m+1}}{2} \frac{(-4)^{\left\lfloor\frac{m+1}{2}\right\rfloor-1}}{\left(\left\lfloor\frac{m+1}{2}\right\rfloor !\right)^{2}} \frac{\left[1-(-1)^{a}\right]\left[1-(-1)^{b}\right]}{(-1)^{\left\lfloor\frac{a-1}{2}\right\rfloor+\left\lfloor\frac{b-1}{2}\right\rfloor}}\left(\begin{array}{c}
\left\lfloor\frac{m+1}{2}\right\rfloor \\
a
\end{array}\right)\left(\begin{array}{c}
\left\lfloor\frac{m+1}{2}\right\rfloor \\
b
\end{array}\right)
$$

for $a, b \in \mathbb{Z}_{\geq 0}$. (It is still understood that empty sums vanish and certain combinatorial expressions are zero.)

Proposition 3.7 (Recursions for $\mathbf{S}_{m}$ ). (a) For $k \in \mathbb{Z}_{>1}$ and $a, b \in \mathbb{Z} \cap[1, k]$, we have

$$
\begin{aligned}
& a(2 k+1-b)\left(\mathbf{S}_{2 k}^{A}\right)_{a, b}=2(2 k+1)\left(\mathbf{S}_{2 k-1}^{A}\right)_{a-1, b}-4\left(\mathbf{S}_{2 k-2}^{A}\right)_{a-1, b-1}, \\
& a(2 k+1-b)\left(\mathbf{S}_{2 k}^{B}\right)_{a, b}=2(2 k+1)\left(\mathbf{S}_{2 k-1}^{B}\right)_{a-1, b}-4\left(\mathbf{S}_{2 k-2}^{B}\right)_{a-1, b-1} .
\end{aligned}
$$

(b) For $k \in \mathbb{Z}_{>1}$, we have

$$
\boldsymbol{\varphi}_{2 k-1}^{-1} \boldsymbol{\alpha}_{k}^{-1} \mathbf{S}_{2 k-1}\left(\boldsymbol{\alpha}_{k}^{-1}\right)^{\mathrm{T}}\left(\boldsymbol{\varphi}_{2 k-1}^{-1}\right)^{\mathrm{T}}=\left(\begin{array}{ll}
\frac{2 k+1}{2} \mathbf{S}_{2 k}^{B} & \\
& -\frac{2}{2 k+1} \mathbf{S}_{2 k-2}^{B}
\end{array}\right)
$$

in block diagonal form, along with a recursion

$$
\frac{4\left(\mathbf{S}_{2 k}^{B}\right)_{a, b}}{(2 k+2-a)(2 k+2-b)}=\left(\mathbf{S}_{\circ}^{B} B+2\right)_{a+1, b+1}-\frac{1+(-1)^{a}}{(-1)^{\left\lfloor\frac{a}{2}\right\rfloor}} \frac{\left(\begin{array}{l}
k+1 \\
a+1
\end{array}\right)\left(\mathbf{S}_{\circ}^{B}\right.}{2 k+2-a}
$$

for $a, b \in \mathbb{Z} \cap[1, k]$. 
Proof. (a) Define

$$
\begin{aligned}
& \mathscr{S}_{m, a, b, s}^{A}:=\frac{\left(\begin{array}{c}
m+1-a \\
\left\lfloor\frac{m+1}{2}\right\rfloor+s
\end{array}\right)\left(\begin{array}{c}
\left\lfloor\frac{m}{2}\right\rfloor+1-s \\
b
\end{array}\right)}{a !(m+1-a) !}=\mathscr{S}_{m, b, a, s}^{A}, \\
& \mathscr{S}_{m, a, b, s}^{B}:=\mathscr{S}_{m, a, b, s}^{A}-\frac{(-1)^{b}\left(\left\lfloor\frac{m+1}{2}\right\rfloor+s\right)}{a !(m+1-a) !}
\end{aligned}
$$

for all the integers $m, a, b, s$ that make these expressions meaningful.

By direct computation, we obtain

$$
a(2 k+1-b) \mathscr{S}_{2 k, a, b, s}^{A}=(2 k+1) \mathscr{S}_{2 k-1, a-1, b, s}^{A}+\mathscr{S}_{2 k-2, a-1, b-1, s}^{A},
$$

for integers $k, a, b, s$ that make each summand well-defined. Multiplying through $(-1)^{s}$ and summing over $s \in \mathbb{Z}_{>1}$, we arrive at the recursion (3.83) that involves

$$
\left(\mathbf{S}_{m}^{A}\right)_{a, b}=\frac{1+(-1)^{a+b+m+1}}{2^{1-m}} \frac{\sum_{s=1}^{\infty}(-1)^{s} \mathscr{S}_{m, a, b, s}^{A}}{(-1)^{\left\lfloor\frac{a+1}{2}\right\rfloor+\left\lfloor\frac{b+1}{2}-\frac{1+(-1)^{m}}{4}\right\rfloor-\left\lfloor\frac{m}{2}\right\rfloor-1}} .
$$

Here, the infinite series over $s$ truncates after finitely many terms, in accordance with the previous definition of $\left(\mathbf{S}_{m}^{A}\right)_{a, b}$ in 1.13 .

Likewise, one can sum over

$$
a(2 k+1-b) \mathscr{S}_{2 k, a, b, s}^{B}=(2 k+1) \mathscr{S}_{2 k-1, a-1, b, s}^{B}+\mathscr{S}_{2 k-2, a-1, b-1, s}^{B}
$$

to arrive at (3.84).

(b) We partition the matrix $\boldsymbol{\alpha}_{k}^{-1} \mathbf{S}_{2 k-1}\left(\boldsymbol{\alpha}_{k}^{-1}\right)^{\mathrm{T}}=\boldsymbol{\alpha}_{k}^{-1}\left(\begin{array}{cc}\mathbf{S}_{2 k-1}^{A} & \mathbf{S}_{2 k-1}^{B} \\ \mathbf{S}_{2 k-1}^{C} & \mathbf{S}_{2 k-1}^{D}\end{array}\right)\left(\boldsymbol{\alpha}_{k}^{-1}\right)^{\mathrm{T}}=\left(\begin{array}{cc}\mathbf{T}_{2 k-1}^{A} & \mathbf{T}_{2 k-1}^{B} \\ \mathbf{T}_{2 k-1}^{C} & \mathbf{T}_{2 k-1}^{D}\end{array}\right)$ into four blocks. Here, the matrix $\mathbf{T}_{2 k-1}^{A}$ also forms the top-left $k \times k$ block of $\boldsymbol{\varphi}_{2 k-1}^{-1} \boldsymbol{\alpha}_{k}^{-1} \mathbf{S}_{2 k-1}\left(\boldsymbol{\alpha}_{k}^{-1}\right)^{\mathrm{T}}\left(\boldsymbol{\varphi}_{2 k-1}^{-1}\right)^{\mathrm{T}}$.

By definition of $\boldsymbol{\alpha}_{k}$ [see (2.23)], we have $\mathbf{T}_{2 k-1}^{D}=\mathbf{S}_{2 k-1}^{D}$.

Thanks to (3.58), we can evaluate the first row and the first column in the top-left $k \times k$ block $\mathbf{T}_{2 k-1}^{A}$ in closed form. This allows us to verify the relations $\left(\mathbf{T}_{2 k-1}^{A}\right)_{1,1}:=\left(\mathbf{S}_{2 k-1}^{A}\right)_{1,1}=\frac{2 k+1}{2}\left(\mathbf{S}_{2 k}^{B}\right)_{1,1}$ and $\left(\mathbf{T}_{2 k-1}^{A}\right)_{1, b} \equiv\left(\mathbf{T}_{2 k-1}^{A}\right)_{b, 1}:=\left(\mathbf{S}_{2 k-1}^{A}\right)_{1, b}+\left(\mathbf{S}_{2 k-1}^{B}\right)_{1, b-1}=\frac{2 k+1}{2}\left(\mathbf{S}_{2 k}^{B}\right)_{1, b}, b \in \mathbb{Z} \cap[2, k]$ explicitly. In general, we may construct a sum rule $\left(\mathbf{S}_{2 k-1}^{A}\right)_{a, b}+\left(\mathbf{S}_{2 k-1}^{B}\right)_{a, b-1}=\frac{a}{2}\left(\mathbf{S}_{2 k}^{B}\right)_{a, b}$ for $a, b \in \mathbb{Z} \cap[2, k]$, using the combinatorial identity $\left(\begin{array}{c}k-s \\ b-1\end{array}\right)+\left(\begin{array}{c}k-s \\ b\end{array}\right)=\left(\begin{array}{c}k+1-s \\ b\end{array}\right)$.

By definition, for $a, b \in \mathbb{Z} \cap[2, k]$, we have $\left(\mathbf{T}_{2 k-1}^{A}\right)_{a, b}:=\left(\mathbf{S}_{2 k-1}^{A}\right)_{a, b}+\left(\mathbf{S}_{2 k-1}^{B}\right)_{a, b-1}+\left(\mathbf{S}_{2 k-1}^{C}\right)_{a-1, b}+$ $\left(\mathbf{S}_{2 k-1}^{D}\right)_{a-1, b-1}$ and $\left(\mathbf{T}_{2 k-1}^{C}\right)_{a-1, b}:=\left(\mathbf{S}_{2 k-1}^{C}\right)_{a-1, b}+\left(\mathbf{S}_{2 k-1}^{D}\right)_{a-1, b-1}$, which together imply $\left(\mathbf{T}_{2 k-1}^{A}\right)_{a, b}=$ $\frac{a}{2}\left(\mathbf{S}_{\circ k}^{B}\right)_{a, b}+\left(\mathbf{T}_{2 k-1}^{C}\right)_{a-1, b}$. In order to identify the last expression with $\frac{2 k+1}{2}\left(\mathbf{S}_{\circ k}^{B}\right)_{a, b}$ [as claimed in the right-hand side of $(3.85)]$, we will prove the relation $\left(\mathbf{T}_{2 k-1}^{C}\right)_{a-1, b}=\frac{2 k+1-a}{2}\left(\mathbf{S}_{0}^{B}\right)_{a, b}$ in the next paragraph.

We aim for an identity that is slightly stronger than what has been just claimed, namely

$$
\left(\mathbf{S}_{\circ}^{B}{ }_{2 k-1}\right)_{a-1, b}+\left(\mathbf{S}_{2 k-1}^{D}\right)_{a, b}=\frac{2 k+1-b}{2}\left(\mathbf{S}_{2 k}^{B}\right)_{a, b}
$$

for all the possible values of $k \in \mathbb{Z}_{>0}$ and $a, b \in \mathbb{Z}$ that make both sides well-defined. For $k=1$, we can check (3.92) by direct computation. The same is true for $a, b \in \mathbb{Z}_{\leq 0}$ and arbitrary $k \in \mathbb{Z}_{>0}$. For larger integer values of $k$, we will verify (3.92) by recursion. Summing over

$$
b(2 k+2-a) \mathscr{S}_{2 k+1, a, b, s}^{A}=(2 k+2) \mathscr{S}_{2 k, a, b-1, s+1}^{A}+\mathscr{S}_{2 k-1, a-1, b-1, s}^{A},
$$

while taking care of contributions from the boundary value (for $s=1$ ) and a combinatorial identity

$$
\sum_{s=1}^{\infty}(-1)^{s}\left(\mathscr{S}_{m, a, b, s}^{B}-\mathscr{S}_{m, a, b, s}^{A}\right)=\frac{\left\lfloor\frac{m+1}{2}\right\rfloor+1-b}{m+1-a-b} \frac{\left(\begin{array}{c}
m+1-a \\
\left\lfloor\frac{m+1}{2}\right\rfloor+1
\end{array}\right)\left(\begin{array}{c}
\left\lfloor\frac{m+1}{2}\right\rfloor+1 \\
b
\end{array}\right)}{(-1)^{b} a !(m+1-a) !},
$$


we reach a result

$$
\begin{aligned}
& b(2 k+2-a)\left(\mathbf{S}_{\circ 2 k+1}^{B}\right)_{a, b}-4(k+1)\left(\mathbf{S}_{2 k}^{B}\right)_{a, b-1}+4\left(\mathbf{S}_{\circ 2 k-1}^{B}\right)_{a-1, b-1} \\
= & (a+1) b\left(\mathbf{S}_{\circ}^{D} D+1\right)_{a+1, b}-4\left(\mathbf{S}_{2 k-1}^{D}\right)_{a, b-1} .
\end{aligned}
$$

We then combine the equation above with (3.84) into a recursion

$$
\begin{aligned}
& (a+1) b\left[\left(\mathbf{S}_{2 k+1}^{B}\right)_{a, b}+\left(\mathbf{S}_{\circ k+1}^{D}\right)_{a+1, b}-\frac{2 k+3-b}{2}\left(\mathbf{S}_{2 k+2}^{B}\right)_{a+1, b}\right] \\
= & 4\left[\left(\mathbf{S}_{\circ 2 k-1}^{B}\right)_{a-1, b-1}+\left(\mathbf{S}_{2 k-1}^{D}\right)_{a, b-1}-\frac{2 k+2-b}{2}\left(\mathbf{S}_{2 k}^{B}\right)_{a, b-1}\right],
\end{aligned}
$$

which proves 3.92 .

The transpose of (3.92) brings us $\frac{2 k+1-a}{2}\left(\mathbf{S}_{2 k}^{B}\right)_{a, b}=\left(\mathbf{T}_{2 k-1}^{C}\right)_{a-1, b}=\frac{2 k+1-a}{2 k+1}\left(\mathbf{T}_{2 k-1}^{A}\right)_{a, b}$ for $a, b \in \mathbb{Z} \cap$ $[2, k]$, while $\left(\mathbf{T}_{2 k-1}^{C}\right)_{a-1,1}=\frac{2 k+1-a}{2 k+1}\left(\mathbf{T}_{2 k-1}^{A}\right)_{a, 1}$ also holds for $a \in \mathbb{Z} \cap[2, k]$. Thus, the top-left, top-right and bottom-left blocks of $\boldsymbol{\varphi}_{2 k-1}^{-1} \boldsymbol{\alpha}_{k}^{-1} \mathbf{S}_{2 k-1}\left(\boldsymbol{\alpha}_{k}^{-1}\right)^{\mathrm{T}}\left(\boldsymbol{\varphi}_{2 k-1}^{-1}\right)^{\mathrm{T}}$ assume the forms described in the righthand side of (3.85).

Eliminating $\left(\mathbf{S}_{2 k-1}^{B}\right)_{a-1, b}$ from (3.84) and (3.92), while noting that $\left(\mathbf{S}_{2 k-1}^{D}\right)_{a, b}=(-1)^{\left\lfloor\frac{a-1}{2}\right\rfloor \frac{1-(-1)^{a}}{2}}$ $\frac{2 k+1-b}{2 k+1}\left(\begin{array}{l}k \\ a\end{array}\right)\left(\mathbf{S}_{\circ}^{B}\right)_{1, b}$, we arrive at (3.86).

With the aid of (3.86), one can verify that the bottom-right $(k-1) \times(k-1)$ block of $\boldsymbol{\varphi}_{2 k-1}^{-1} \boldsymbol{\alpha}_{k}^{-1} \mathbf{S}_{2 k-1}$ $\left(\boldsymbol{\alpha}_{k}^{-1}\right)^{\mathrm{T}}\left(\boldsymbol{\varphi}_{2 k-1}^{-1}\right)^{\mathrm{T}}$ is indeed equal to $-\frac{2}{2 k+1} \mathbf{S}_{2 k-2}^{B}$.

\section{BROADHURST-ROBERTS QUADRATIC RELATIONS}

In $\$ 4.1$, we characterize $\mathbf{S}_{m}^{-1}$ in terms of Bernoulli numbers, thereby connecting sum rules of Feynman diagrams to the Betti matrix $\mathbf{B}_{m-2}$ in the on-shell limit. In the meantime, we will also represent the de Rham matrix $\mathbf{D}_{m-2}$ for Broadhurst-Roberts quadratic relations in the form declared in Theorem 1.2.

In $\$ 4.2$, we explore the Broadhurst-Roberts varieties $V_{m+2}^{\mathrm{BR}}:=\left\{\mathbf{X}_{\left\lfloor\frac{m+1}{2}\right\rfloor} \in \mathbb{C}^{\left\lfloor\frac{m+1}{2}\right\rfloor \times\left\lfloor\frac{m+1}{2}\right\rfloor} \mid \mathbf{X}_{\left\lfloor\frac{m+1}{2}\right\rfloor} \mathbf{D}_{m} \mathbf{X}_{\left\lfloor\frac{m+1}{2}\right\rfloor}^{\mathrm{T}}-\right.$ $\left.\mathbf{B}_{m}=0\right\}$, replacing the Broadhurst-Roberts period matrices $\mathbf{P}_{m}$ with formal variables. We demonstrate algebraically that $V_{2 k+1}^{\mathrm{BR}}$ imposes a constraint on certain minor determinants of $\mathbf{X}_{k}$, in the form of a reflection formula (1.21).

The purpose of $\$ 4.3$ is twofold. First, we display the diversity of de Rham representations in the Wrońskian framework, along with formal proofs. Second, we conclude with an open-ended discussion on other representations of the de Rham matrix $\mathbf{D}_{m}$ outside the Wrońskian framework.

4.1. Quadratic relations for on-shell Bessel moments. It is now clear from Proposition 2.7 that

$$
\left|\mathcal{L}_{2 k-1}(1)\right| \boldsymbol{\alpha}_{k}^{\mathrm{T}} \mathbf{W}_{2 k-1}^{\mathrm{T}}(1) \mathbf{V}_{2 k-1}(1) \mathbf{W}_{2 k-1}(1) \boldsymbol{\alpha}_{k}=\boldsymbol{\alpha}_{k}^{\mathrm{T}} \mathbf{S}_{2 k-1}^{-1} \boldsymbol{\alpha}_{k}
$$

can be rewritten as

$$
\frac{\boldsymbol{\alpha}_{k}^{\mathrm{T}} \mathbf{S}_{2 k-1}^{-1} \boldsymbol{\alpha}_{k}}{\left|\mathcal{L}_{2 k-1}(1)\right|}=\left(\begin{array}{rr}
\mathbf{P}_{2 k-1} & \dot{\mathbf{P}}_{2 k-1} \\
& -\mathbf{P}_{2 k-3}
\end{array}\right)\left[\left(\boldsymbol{\beta}_{2 k-1}^{-1}\right)^{\mathrm{T}} \mathbf{V}_{2 k-1}(1) \boldsymbol{\beta}_{2 k-1}^{-1}\right]\left(\begin{array}{ll}
\mathbf{P}_{2 k-1}^{\mathrm{T}} & \\
\hat{\mathbf{P}}_{2 k-1}^{\mathrm{T}} & -\mathbf{P}_{2 k-3}^{\mathrm{T}}
\end{array}\right),
$$

whose bottom-right $(k-1) \times(k-1)$ block is partly responsible for Theorem $1.2[m=2 k-1$ in (1.20) and (1.22)]. Proposition 2.8 then brings us a further refinement:

$$
\frac{\boldsymbol{\varphi}_{2 k-1}^{\mathrm{T}} \boldsymbol{\alpha}_{k}^{\mathrm{T}} \mathbf{S}_{2 k-1}^{-1} \boldsymbol{\alpha}_{k} \boldsymbol{\varphi}_{2 k-1}}{\left|\mathcal{L}_{2 k-1}(1)\right|}=\left(\begin{array}{ll}
\mathbf{P}_{2 k-1} & \\
& -\mathbf{P}_{2 k-3}
\end{array}\right)\left[\left(\boldsymbol{\vartheta}_{2 k-1}^{-1}\right)^{\mathrm{T}}\left(\boldsymbol{\beta}_{2 k-1}^{-1}\right)^{\mathrm{T}} \mathbf{V}_{2 k-1}(1) \boldsymbol{\beta}_{2 k-1}^{-1} \boldsymbol{\vartheta}_{2 k-1}^{-1}\right]\left(\begin{array}{ll}
\mathbf{P}_{2 k-1}^{\mathrm{T}} & \\
& -\mathbf{P}_{2 k-1}^{\mathrm{T}}
\end{array}\right) .
$$


Here, the bottom right $(k-1) \times(k-1)$ block in $\boldsymbol{\alpha}_{k}^{\mathrm{T}} \mathbf{S}_{2 k-1}^{-1} \boldsymbol{\alpha}_{k}\left[\operatorname{resp} .\left(\boldsymbol{\beta}_{2 k-1}^{-1}\right)^{\mathrm{T}} \mathbf{V}_{2 k-1}(1) \boldsymbol{\beta}_{2 k-1}\right]$ is unaffected by left multiplication of $\boldsymbol{\varphi}_{2 k-1}^{\mathrm{T}}\left[\right.$ resp. $\left.\left(\boldsymbol{\vartheta}_{2 k-1}^{-1}\right)^{\mathrm{T}}\right]$ and right multiplication of $\boldsymbol{\varphi}_{2 k-1}$ (resp. $\left.\boldsymbol{\vartheta}_{2 k-1}^{-1}\right)$.

To obtain similar block (tri)diagonalization for Theorem $1.2(m=2 k)$, we need to expend a little more effort, in the lemma below.

Lemma 4.1 (Margin behavior). For each given $k \in \mathbb{Z}_{>0}$, the $2 k$-th row and the $2 k$-th column of

$$
\lim _{u \rightarrow 1^{-}}\left|\mathcal{L}_{2 k}(u)\right|\left(\boldsymbol{\beta}_{2 k}^{-1}\right)^{\mathrm{T}} \mathbf{V}_{2 k}(u) \boldsymbol{\beta}_{2 k}^{-1}
$$

are filled with zeros.

Proof. We first note that

$$
\begin{aligned}
\left(D^{0} I_{0}(\sqrt{u} t), \ldots, D^{2 k-1} I_{0}(\sqrt{u} t)\right)^{\mathrm{T}}= & {\left[\boldsymbol{\beta}_{2 k}(u)\right]^{-1}\left(I_{0}(\sqrt{u} t),-I_{0}(\sqrt{u} t) t^{2} \ldots,(-1)^{k-1} I_{0}(\sqrt{u} t) t^{2 k-2},\right.} \\
& \left.\sqrt{u} I_{1}(\sqrt{u} t) t,-\sqrt{u} I_{1}(\sqrt{u} t) t^{3} \ldots,(-1)^{k-1} \sqrt{u} I_{1}(\sqrt{u} t) t^{2 k-1}\right)^{\mathrm{T}}
\end{aligned}
$$

entails a characterization of the last column in $\boldsymbol{\beta}_{2 k}^{-1}$ :

$$
\left(\boldsymbol{\beta}_{2 k}^{-1}\right)_{a, 2 k}=\frac{(-1)^{k-1}}{2^{2 k-1}} \delta_{a, 2 k}
$$

Then we observe that $\left(\mathbf{V}_{2 k}(u) \boldsymbol{\beta}_{2 k}^{-1}\right)_{a, 2 k}=\sum_{t=1}^{2 k}\left(\mathbf{V}_{2 k}(u)\right)_{a, t} \frac{(-1)^{k-1}}{2^{2 k-1}} \delta_{t, 2 k}=\frac{(-1)^{k-1}}{2^{2 k-1}}\left(\mathbf{V}_{2 k}(u)\right)_{a, 2 k}=\frac{(-1)^{k-1}}{2^{2 k-1}} \delta_{a, 1}$ and $\left(\left(\boldsymbol{\beta}_{2 k}^{-1}\right)^{\mathrm{T}} \mathbf{V}_{2 k}(u) \boldsymbol{\beta}_{2 k}\right)_{a, 2 k}=\frac{(-1)^{k-1}}{2^{2 k-1}} \delta_{a, 1}$ follow immediately. By skew symmetry, we have $\left(\left(\boldsymbol{\beta}_{2 k}^{-1}\right)^{\mathrm{T}} \mathbf{V}_{2 k}(u) \boldsymbol{\beta}_{2 k}\right)_{2 k, b}=$ $\frac{(-1)^{k}}{2^{2 k-1}} \delta_{1, b}$, so the right and bottom margins of the matrix $\lim _{u \rightarrow 1^{-}}\left|\mathcal{L}_{2 k}(u)\right|\left(\boldsymbol{\beta}_{2 k}^{-1}\right)^{\mathrm{T}} \mathbf{V}_{2 k}(u) \boldsymbol{\beta}_{2 k}$ are indeed filled with zeros.

According to the result from the last lemma, we have (cf. Proposition 2.77)

$$
\begin{aligned}
& \boldsymbol{\alpha}_{k}^{\mathrm{T}} \boldsymbol{\psi}_{k}^{\mathrm{T}} \mathbf{S}_{2 k}^{-1} \boldsymbol{\psi}_{k} \boldsymbol{\alpha}_{k} \\
= & \lim _{u \rightarrow 1^{-}}\left|\mathcal{L}_{2 k}(u)\right| \boldsymbol{\alpha}_{k}^{\mathrm{T}} \boldsymbol{\psi}_{k}^{\mathrm{T}} \mathbf{W}_{2 k}^{\mathrm{T}}(u) \mathbf{V}_{2 k}(u) \mathbf{W}_{2 k}(u) \boldsymbol{\psi}_{k} \boldsymbol{\alpha}_{k} \\
= & \left(\begin{array}{rr}
\mathbf{P}_{2 k} & \dot{\mathbf{P}}_{2 k} \\
& -\mathbf{P}_{2 k-2}
\end{array}\right)\left[\lim _{u \rightarrow 1^{-}}\left|\mathcal{L}_{2 k}(u)\right| \boldsymbol{\varrho}_{k}\left(\boldsymbol{\beta}_{2 k}^{-1}\right)^{\mathrm{T}} \mathbf{V}_{2 k}(u) \boldsymbol{\beta}_{2 k}^{-1} \boldsymbol{\varrho}_{k}^{\mathrm{T}}\right]\left(\begin{array}{ll}
\mathbf{P}_{2 k}^{\mathrm{T}} & \\
\hat{\mathbf{P}}_{2 k}^{\mathrm{T}} & -\mathbf{P}_{2 k-2}^{\mathrm{T}}
\end{array}\right),
\end{aligned}
$$

which is partly responsible for Theorem $1.2[m=2 k$ in $[1.20)$ and (1.22)]. Its block diagonalized counterpart reads (cf. Proposition 2.8)

$$
\begin{aligned}
& \boldsymbol{\varphi}_{2 k}^{\mathrm{T}} \boldsymbol{\alpha}_{k}^{\mathrm{T}} \boldsymbol{\psi}_{k}^{\mathrm{T}} \mathbf{S}_{2 k}^{-1} \boldsymbol{\psi}_{k} \boldsymbol{\alpha}_{k} \boldsymbol{\varphi}_{2 k} \\
= & \left(\begin{array}{cc}
\mathbf{P}_{2 k} & \\
& -\mathbf{P}_{2 k-2}
\end{array}\right)\left(\boldsymbol{\vartheta}_{2 k}^{-1}\right)^{\mathrm{T}}\left[\lim _{u \rightarrow 1^{-}}\left|\mathcal{L}_{2 k}(u)\right| \boldsymbol{\varrho}_{k}\left(\boldsymbol{\beta}_{2 k}^{-1}\right)^{\mathrm{T}} \mathbf{V}_{2 k}(u) \boldsymbol{\beta}_{2 k}^{-1} \boldsymbol{\varrho}_{k}^{\mathrm{T}}\right] \boldsymbol{\vartheta}_{2 k}^{-1}\left(\begin{array}{cc}
\mathbf{P}_{2 k}^{\mathrm{T}} & \\
& -\mathbf{P}_{2 k-2}^{\mathrm{T}}
\end{array}\right) .
\end{aligned}
$$

To complete the proof of Theorem 1.2, we need to express the entries of $\mathbf{S}_{m}^{-1}$ via Bernoulli numbers, in the next proposition.

Proposition 4.2 (Bernoulli representation of $\mathbf{S}_{m}^{-1}$ ). Let $\mathrm{B}_{n}$ be the Bernoulli numbers generated by (1.20).

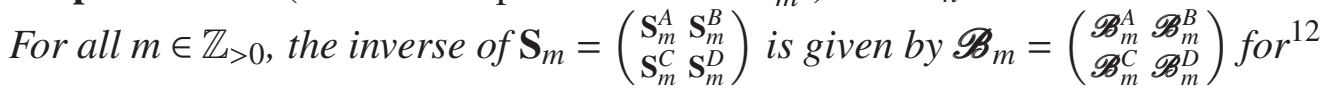

$$
\left(\mathscr{B}_{m}^{A}\right)_{a, b}:=\frac{(m+1) !}{m+2} \frac{\delta_{\min \{a, b\}, 1} \mathrm{~B}_{m+3-a-b}}{2^{m-1}(-1)^{\left\lfloor\frac{m+2-a-b}{2}\right\rfloor+\frac{1+a-(-1)^{m}(1-a)}{2}+\left\lfloor\frac{m+1}{2}\right\rfloor}}
$$

\footnotetext{
${ }^{12}$ It is understood that $\left(\mathbf{S}_{1}\right)_{1,1}=9,\left(\mathscr{B}_{1}\right)_{1,1}=\frac{1}{9}$, and $\mathbf{S}_{2}=\mathbf{S}_{2}^{A}=\left(\mathscr{B}_{2}\right)^{-1}=\left(\mathscr{B}_{2}^{A}\right)^{-1}=\left(\begin{array}{cc}0 & -8 \\ 8 & 0\end{array}\right)$.
} 
where $a, b \in \mathbb{Z} \cap\left[1,\left\lfloor\frac{m}{2}\right\rfloor+1\right]$,

$$
\left(\mathscr{B}_{m}^{B}\right)_{a, b^{\prime}}=(-1)^{m+1}\left(\mathscr{B}_{m}^{C}\right)_{b^{\prime}, a}:=\frac{(m+2-a) !\left(m-b^{\prime}\right) !}{\left(m+2-a-b^{\prime}\right) !} \frac{\left(1-\frac{a+b^{\prime}}{m+2} \delta_{a, 1}\right) \mathrm{B}_{m+2-a-b^{\prime}}}{2^{m-1}(-1)^{\left\lfloor\frac{m+1-a-b^{\prime}}{2}\right\rfloor+b^{\prime}+\left\lfloor\frac{m}{2}\right\rfloor}}
$$

where $a \in \mathbb{Z} \cap\left[1,\left\lfloor\frac{m}{2}\right\rfloor+1\right], b^{\prime} \in \mathbb{Z} \cap\left[1,\left\lfloor\frac{m-1}{2}\right\rfloor\right]$, and

$$
\left(\mathscr{B}_{m}^{D}\right)_{a^{\prime}, b^{\prime}}:=\frac{\left(m-a^{\prime}\right) !\left(m-b^{\prime}\right) !}{\left(m+1-a^{\prime}-b^{\prime}\right) !} \frac{\left(m-a^{\prime}-b^{\prime}\right) \mathrm{B}_{m+1-a^{\prime}-b^{\prime}}}{2^{m-1}(-1)^{\left\lfloor\frac{m-a^{\prime}-b^{\prime}}{2}\right\rfloor+a^{\prime}+\left\lfloor\frac{m-1}{2}\right\rfloor}}
$$

where $a^{\prime}, b^{\prime} \in \mathbb{Z} \cap\left[1,\left\lfloor\frac{m-1}{2}\right\rfloor\right]$.

Consequently, both (4.2) and (4.2) [resp. (4.6) and (4.6)] account for the representations of the de Rham matrix $\mathbf{D}_{2 k-3}$ [resp. $\mathbf{D}_{2 k-2}$ ] per (1.17) and the Betti matrix $\mathbf{B}_{2 k-3}$ [resp. $\mathbf{B}_{2 k-2}$ ] per (1.19).

Proof. By direct computation, one can check that

$$
\boldsymbol{\varphi}_{2 k-1}^{\mathrm{T}} \boldsymbol{\alpha}_{k}^{\mathrm{T}} \mathscr{B}_{2 k-1} \boldsymbol{\alpha}_{k} \boldsymbol{\varphi}_{2 k-1}=\left(\begin{array}{cc}
\frac{2^{4}(-1)^{k-1}}{2 k+1} \mathbf{B}_{2 k-1} & \\
& 2^{2}(2 k+1)(-1)^{k-1} \mathbf{B}_{2 k-3}
\end{array}\right),
$$

and

$$
\boldsymbol{\varphi}_{2 k}^{\mathrm{T}} \boldsymbol{\alpha}_{k}^{\mathrm{T}} \boldsymbol{\psi}_{k}^{\mathrm{T}} \mathscr{B}_{2 k} \boldsymbol{\Psi}_{k} \boldsymbol{\alpha}_{k} \boldsymbol{\varphi}_{2 k}=\left(\begin{array}{ll}
\frac{2^{4}(-1)^{k-1}}{2 k+2} \mathbf{B}_{2 k} & \\
& 2^{2}(2 k+2)(-1)^{k-1} \mathbf{B}_{2 k-2}
\end{array}\right),
$$

where the entries of $\mathbf{B}_{m-2} \in \mathbb{Q}^{\left\lfloor\frac{\lfloor-1}{2}\right\rfloor \times\left\lfloor\frac{m-1}{2}\right\rfloor}$ are given by the last line of (1.19). (This is essentially the original form of Betti matrix conjectured by Broadhurst-Roberts [13, (5.4)], except that all its rows and columns are placed in reverse order.) Therefore, so long as one can verify that $\mathbf{S}_{m}^{-1}=\mathscr{B}_{m}$ for $m \in \mathbb{Z}_{>1}$, one can deduce all the Broadhurst-Roberts quadratic relations $\mathbf{P}_{m} \mathbf{D}_{m} \mathbf{P}_{m}^{\mathrm{T}}=\mathbf{B}_{m}$ from the bottom-right blocks of 4.2 and 4.6 .

Recall the reshuffling-rescaling matrix $\boldsymbol{\xi}_{m}$ from (3.81). In the block partition of $\mathscr{B}_{m}:=\boldsymbol{\xi}_{m} \mathscr{B}_{m} \boldsymbol{\xi}_{m}^{\mathrm{T}}=$ $\left(\begin{array}{r}\mathscr{B}_{m}^{B} \\ \mathscr{B}_{m}^{C} \mathscr{B}_{0}^{D}\end{array}\right)$, the top-left $\left\lfloor\frac{m}{2}\right\rfloor \times\left\lfloor\frac{m}{2}\right\rfloor$ block is filled with zeros, while the remaining blocks are $\left(\mathscr{B}_{m}^{B}\right)_{a, b}=$ $(-1)^{m+1}\left(\mathscr{B}_{\circ}^{C}\right)_{b, a}=\left(\mathscr{B}_{m}^{B}\right)_{a+1, b-1}$ for $a \in \mathbb{Z} \cap\left[1,\left\lfloor\frac{m}{2}\right\rfloor\right], b \in \mathbb{Z} \cap\left[1,\left\lfloor\frac{m+1}{2}\right\rfloor\right]$ and

$$
\left(\mathscr{B}_{0}^{D}\right)_{a, b}=\left(\mathscr{B}_{m}^{D}\right)_{a-1, b-1}\left(1+\frac{\delta_{a, 1}+\delta_{b, 1}}{m+2-a-b}\right)
$$

for $a, b \in \mathbb{Z} \cap\left[1,\left\lfloor\frac{m+1}{2}\right\rfloor\right]$. Here, expressions like $\left(\mathscr{B}_{m}^{B}\right)_{a+1,0},\left(\mathscr{B}_{m}^{D}\right)_{0, b-1}$ and $\left(\mathscr{B}_{m}^{D}\right)_{a-1,0}$ are defined by the right-hand sides of (4.8) and (4.9).

In what follows, we will demonstrate inductively that

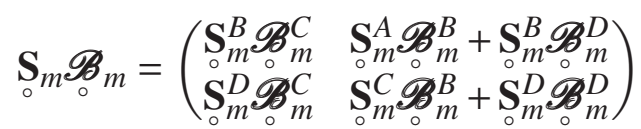

is the $m \times m$ identity matrix $\mathbf{I}_{m}$, for all positive integers $m$. Suppose that we have verified the aforementioned claim up to a certain odd number $m=2 k-1$. We will show that the same is true for $\mathbf{S}_{2 k} \mathscr{B}_{2 k}$ and $\mathbf{S}_{2 k+1} \mathscr{B}_{2 k+1}$. Inductive evaluation of $\mathbf{S}_{2 k} \mathscr{B}_{2 k}$

We note that $\mathbf{S}_{2 k}^{D}$ is filled with zeros. So must be the case for $\mathbf{S}_{2 k}^{D} \mathscr{B}_{2 k}^{C}$, which forms the bottom-left block of $\mathbf{S}_{2 k} \mathscr{B}_{2 k}$.

Paraphrasing our induction hypothesis $\mathbf{S}_{2 k-1}^{-1}=\mathscr{B}_{2 k-1}$ with (3.85) and (4.10), we have $\left(\mathbf{S}_{2 k}^{B}\right)^{-1}=$ $2^{3}(-1)^{k-1} \mathbf{B}_{2 k-1}$. Since $\mathscr{B}_{2 k}^{C}=2^{3}(-1)^{k-1} \mathbf{B}_{2 k-1}$, we can check that the top-left block satisfies $\mathbf{S}_{2 k}^{B} \mathscr{B}_{\circ}^{C}=\mathbf{I}_{k}$ and the bottom-right block has the same behavior: $\mathbf{S}_{2 k}^{C} \mathscr{B}_{2 k}^{B}+\mathbf{S}_{2 k}^{D} \mathscr{B}_{2 k}^{D}=\left(\mathscr{B}_{0}^{C} \mathbf{S}_{2 k}^{B}\right)^{\mathrm{T}}=\mathbf{I}_{k}$. 
In the next three paragraphs, we show that the top-right block $\mathbf{S}_{2 k}^{A} \mathscr{B}_{2 k}^{B}+\mathbf{S}_{2 k}^{B} \mathscr{B}_{2 k}^{D}$ has vanishing entries. This task can be simplified by a similarity transformation

$$
\boldsymbol{\xi}_{2 k}^{\mathrm{T}} \mathbf{S}_{2 k} \mathscr{B}_{2 k}\left(\boldsymbol{\xi}_{2 k}^{\mathrm{T}}\right)^{-1}=\mathbf{S}_{2 k} \mathscr{B}_{2 k}=\left(\begin{array}{lr}
\mathbf{S}_{2 k}^{A} \mathscr{B}_{2 k}^{A}+\mathbf{S}_{2 k}^{B} \mathscr{B}_{2 k}^{C} & \mathbf{S}_{2 k}^{A} \mathscr{B}_{2 k}^{B}+\mathbf{S}_{2 k}^{B} \mathscr{B}_{2 k}^{D} \\
\mathbf{I}_{k}
\end{array}\right) .
$$

Here, the only possible exceptions to $\left(\mathbf{S}_{2 k} \mathscr{B}_{2 k}\right)_{p, q}=\delta_{p, q}$ occur at positions $p \in \mathbb{Z} \cap[2, k+1], q \in \mathbb{Z} \cap$ $(\{1\} \cup[k+2,2 k])$, where we have $\left(\mathbf{S}_{2 k}^{A} \mathscr{B}_{2 k}^{A}+\mathbf{S}_{2 k}^{B} \mathscr{B}_{2 k}^{C}\right)_{a+1,1}=\frac{2 k+1}{2 k+2}\left(\mathbf{S}_{2 k}^{A} \mathscr{B}_{2 k}^{B}+\mathbf{S}_{2 k}^{B} \mathscr{B}_{2 k}^{D}\right)_{a, 1}$ for $a \in \mathbb{Z} \cap[1, k]$ and $\left(\mathbf{S}_{2 k}^{A} \mathscr{B}_{2 k}^{B}+\mathbf{S}_{2 k}^{B} \mathscr{B}_{2 k}^{D}\right)_{a+1, b-1}=\left(\mathbf{S}_{2 k}^{A} \mathscr{B}_{2 k}^{B}+\mathbf{S}_{2 k}^{B} \mathscr{B}_{2 k}^{D}\right)_{a, b}$ for $a \in \mathbb{Z} \cap[1, k], b \in \mathbb{Z} \cap[2, k]$.

First, we show that $\left(\mathbf{S}_{2 k}^{A} \mathscr{B}_{2 k}^{B}+\mathbf{S}_{2 k}^{B} \mathscr{B}_{2 k}^{D}\right)_{a+1, b-1}=0$ for $a \in \mathbb{Z} \cap[1, k]$ and $b \in \mathbb{Z} \cap[3, k]$. Contracting the recursions $\left(\mathscr{B}_{2 k}^{B}\right)_{n+1, b-1}=\frac{2 k+1-n}{2} \frac{1+2 k \delta_{n, 0}}{1+(2 k+1) \delta_{n, 0}}\left(\mathscr{B}_{2 k-1}^{B}\right)_{n+1, b-2}$ for $n \in \mathbb{Z} \cap[0, k], b \in \mathbb{Z} \cap[3, k]$ and $\left(\mathscr{B}_{2 k}^{B}\right)_{n+1, b-1}=\frac{\left[2 k+(1-b)\left(1-\delta_{n, 1}\right)\right](2 k+1-n)}{4}\left(\mathscr{B}_{2 k-2}^{B}\right)_{n, b-2}$ for $n \in \mathbb{Z} \cap[1, k], b \in \mathbb{Z} \cap[3, k]$ with a variation on (3.83), namely

$$
\frac{a(2 k+1-n)\left(\mathbf{S}_{2 k}^{A}\right)_{a+1, n+1}}{1+(2 k+1) \delta_{n, 0}}=\frac{2(2 k+1)\left(\mathbf{S}_{2 k-1}^{A}\right)_{a, n+1}}{\left(1+2 k \delta_{a, 1}\right)\left(1+2 k \delta_{n, 0}\right)}-\frac{4\left(\mathbf{S}_{2 k-2}^{A}\right)_{a, n}}{\left[1+(2 k-1) \delta_{a, 1}\right]\left[1+(2 k-1) \delta_{n, 1}\right]}
$$

for $a \in \mathbb{Z} \cap[1, k], n \in \mathbb{Z} \cap[0, k]$, we compute

$$
\begin{aligned}
\left(\mathbf{S}_{2 k}^{A} \mathscr{B}_{2 k}^{B}\right)_{a+1, b-1}= & \frac{2 k+1}{a} \frac{\left(\mathbf{S}_{2 k-1}^{A} \mathscr{B}_{2 k-1}^{B}\right)_{a, b-2}}{1+2 k \delta_{a, 1}}-\frac{2 k+1-b}{a} \frac{\left(\mathbf{S}_{2 k-2}^{A} \mathscr{B}_{2 k-2}^{B}\right)_{a, b-2}}{1+(2 k-1) \delta_{a, 1}} \\
& +\frac{2 k-b}{a} \frac{\left(\mathbf{S}_{2 k-2}^{A}\right)_{a, 1}\left(\mathscr{B}_{2 k-2}^{B}\right)_{1, b-2}}{1+(2 k-1) \delta_{a, 1}}
\end{aligned}
$$

for $a \in \mathbb{Z} \cap[1, k], b \in \mathbb{Z} \cap[3, k]$, where we have exploited the facts that $\left(\mathbf{S}_{2 k-1}^{A}\right)_{a, k+1}=\left(\mathbf{S}_{2 k-2}^{A}\right)_{a, 0}=0$ by extensions of (1.13)]. Likewise, we can argue that

$$
\begin{aligned}
\left(\mathbf{S}_{2 k}^{B} \mathscr{B}_{2 k}^{D}\right)_{a+1, b-1}= & \frac{2 k+1}{a} \frac{\left(\mathbf{S}_{2 k-1}^{B} \mathscr{B}_{2 k-1}^{D}\right)_{a, b-2}}{1+2 k \delta_{a, 1}}-\frac{2 k+1-b}{a} \frac{\left(\mathbf{S}_{2 k-2}^{B} \mathscr{B}_{2 k-2}^{D}\right)_{a, b-2}}{1+(2 k-1) \delta_{a, 1}} \\
& -\frac{2 k+1-b}{a} \frac{\left(\mathbf{S}_{2 k-2}^{B}\right)_{a, 0}\left(\mathscr{B}_{2 k-2}^{D}\right)_{0, b-2}}{1+(2 k-1) \delta_{a, 1}}
\end{aligned}
$$

for $a \in \mathbb{Z} \cap[1, k], b \in \mathbb{Z} \cap[3, k]$. Adding up (4.15) and (4.16), while falling back on our induction hypothesis that $\mathbf{S}_{2 k-1}^{-1}=\mathscr{B}_{2 k-1}$ and $\mathbf{S}_{2 k-2}^{-1}=\mathscr{B}_{2 k-2}$, we arrive at $\left(\mathbf{S}_{2 k}^{A} \mathscr{B}_{2 k}^{B}+\mathbf{S}_{2 k}^{B} \mathscr{B}_{2 k}^{D}\right)_{a+1, b-1}=\left(\mathbf{S}_{2 k}^{A} \mathscr{B}_{2 k}^{B}+\right.$ $\left.\mathbf{S}_{2 k}^{B} \mathscr{B}_{2 k}^{D}\right)_{a, b}=\frac{2 k+1}{a\left(1+2 k \delta_{a, 1}\right)}\left(\mathbf{S}_{2 k}^{A} \mathscr{B}_{2 k}^{B}+\mathbf{S}_{2 k}^{B} \mathscr{B}_{2 k}^{D}\right)_{a, b-2}-\frac{2 k+1-b}{a\left[1+(2 k-1) \delta_{a, 1}\right]}\left(\mathbf{S}_{2 k-2}^{A} \mathscr{B}_{2 k-2}^{B}+\mathbf{S}_{2 k-2}^{B} \mathscr{B}_{2 k-2}^{D}\right)_{a, b-2}=0$ for $a \in \mathbb{Z} \cap[1, k], b \in \mathbb{Z} \cap[3, k]$.

Second, we prove that $\left(\mathbf{S}_{2 k}^{A} \mathscr{B}_{2 k}^{B}+\mathbf{S}_{2 k}^{B} \mathscr{B}_{2 k}^{D}\right)_{2,1}=0$. Adding up analogs of (4.15) and (4.16) for $a=1, b=$ 2, we obtain

$$
\begin{aligned}
\left(\mathbf{S}_{2 k}^{A} \mathscr{B}_{2 k}^{B}+\mathbf{S}_{2 k}^{B} \mathscr{B}_{2 k}^{D}\right)_{2,1}= & \sum_{n=1}^{k}\left[\left(\mathbf{S}_{2 k-1}^{A}\right)_{1, n}\left(\mathscr{B}_{2 k-1}^{B}\right)_{n, 0}+\left(\mathbf{S}_{2 k-1}^{B}\right)_{1, n}\left(\mathscr{B}_{2 k-1}^{D}\right)_{n, 0}\right] \\
& -\frac{2 k-1}{2 k} \sum_{n=2}^{k}\left[\left(\mathbf{S}_{2 k-2}^{A}\right)_{1, n}\left(\mathscr{B}_{2 k-2}^{B}\right)_{n, 0}+\left(\mathbf{S}_{2 k-2}^{B}\right)_{1, n}\left(\mathscr{B}_{2 k-2}^{D}\right)_{n, 0}\right] .
\end{aligned}
$$

Through the explicit formula in (3.58), one can check that $\left(\mathbf{S}_{2 k-1}^{B}\right)_{1, k}=0$ and $\left(\mathbf{S}_{2 k-1}^{A}\right)_{1, n}\left(\mathscr{B}_{2 k-1}^{B}\right)_{n, 0}+$ $\left(\mathbf{S}_{2 k-1}^{B}\right)_{1, n-1}\left(\mathscr{B}_{2 k-1}^{D}\right)_{n-1,0}\left(1-\delta_{n, 1}\right)=\left(\mathbf{S}_{2 k}^{B}\right)_{1, n}\left(\mathscr{B}_{2 k}^{C}\right)_{n, 1}$ for $n \in \mathbb{Z} \cap[1, k]$, so the first displayed sum in (4.17) evaluates to $\left(\mathbf{S}_{2 k}^{B} \mathscr{B}_{2 k}^{C}\right)_{1,1}=1$; referring back to $(3.61)$, one sees that $\left(\mathbf{S}_{2 k-2}^{B}\right)_{1, n}=0$ and $\left(\mathbf{S}_{2 k-2}^{A}\right)_{1, n}\left(\mathscr{B}_{2 k-2}^{B}\right)_{n, 0}=$ $\frac{2 k}{2 k-1}\left(\mathbf{S}_{2 k-2}^{B}\right)_{1, n-1}\left(\mathscr{B}_{\circ}^{C}{ }_{2 k-1}\right)_{n-1,1}$ for $n \in \mathbb{Z} \cap[2, k]$, so the second displayed sum in (4.17) evaluates to $\frac{2 k}{2 k-1}$ $\left(\mathbf{S}_{2 k-2}^{B} \mathscr{B}_{2 k-2}^{C}\right)_{1,1}=\frac{2 k}{2 k-1}$. Thus, the claimed identity is a consequence of our induction hypothesis.

Third, we demonstrate that $\left(\mathbf{S}_{2 k}^{A} \mathscr{B}_{2 k}^{B}+\mathbf{S}_{2 k}^{B} \mathscr{B}_{2 k}^{D}\right)_{a, b}=0$ for $a \in \mathbb{Z} \cap[1, k], b \in\{1,2\}$. If the integers $a$ and $b$ have the same parity, then $\left(\mathbf{S}_{2 k}^{\AA}\right)_{a, n}\left(\mathscr{B}_{2 k}^{B}\right)_{n, b}=\left(\mathbf{S}_{2 k}^{B}\right)_{a, n}\left(\mathscr{B}_{2 k}^{D}\right)_{n, b}=0$ for all $n \in \mathbb{Z} \cap[1, k]$. Thus, we have $\left(\mathbf{S}_{2 k}^{A} \mathscr{B}_{2 k}^{A}+\mathbf{S}_{2 k}^{B} \mathscr{B}_{2 k}^{C}\right)_{2,1}=\frac{2 k+1}{2 k+2}\left(\mathbf{S}_{2 k}^{A} \mathscr{B}_{2 k}^{B}+\mathbf{S}_{0}^{B} \mathscr{B}_{2 k}^{D}\right)_{1,1}=0$. Now we have a matrix partition $\mathbf{S}_{2 k} \mathscr{B}_{2 k}=$ 
$\left(\begin{array}{ccc}\mathbf{I}_{2} & & \\ \mathbf{U} & \mathbf{I}_{k-1} & \mathbf{V} \\ & \mathbf{I}_{k-1}\end{array}\right)$ where all the possibly non-vanishing entries of $\mathbf{U}$ and $\mathbf{V}$ are confined to their first columns. Such a matrix partition is invertible, and $\mathbf{S}_{2 k}^{-1}=\mathscr{B}_{2 k}\left(\begin{array}{ccc}\mathbf{I}_{2} & \\ -\mathbf{U} & \mathbf{I}_{k-1} & -\mathbf{V} \\ & \mathbf{I}_{k-1}\end{array}\right)$ is skew-symmetric. With a matrix

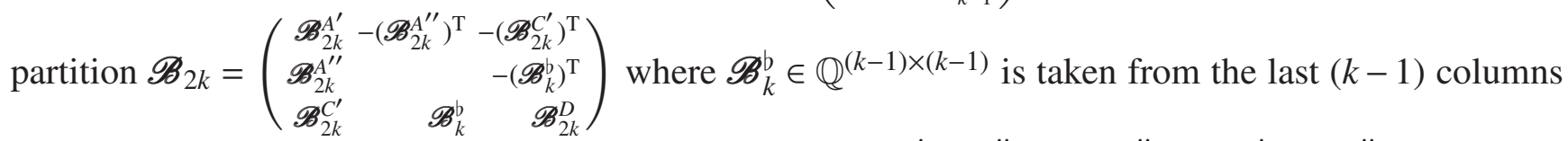

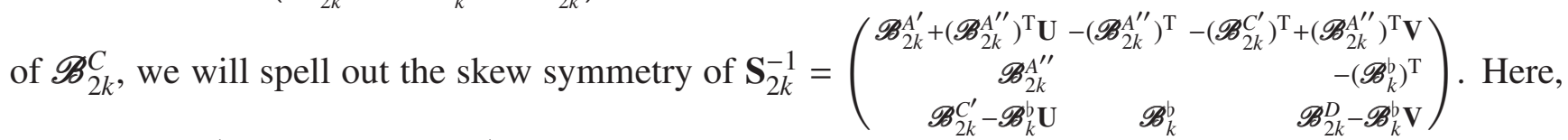
the matrix $\mathscr{B}_{k}^{b} \mathbf{V}$ is equal to $\mathscr{B}_{k}^{b} \mathbf{v}$ followed by the $(k-2)$ identically vanishing columns to the right, where $\mathbf{v}$ is the first column of $\mathbf{V}$. In order to guarantee the skew symmetry of $\mathscr{B}_{k}^{b} \mathbf{V}$, one has to have $\mathscr{B}_{k}^{b} \mathbf{v}=\mathbf{0} \in \mathbb{R}^{k-1}$. We claim that $\operatorname{det} \mathscr{B}_{k}^{b} \neq 0$, which can be seen from the relation between

$$
\left|\operatorname{det} \mathscr{B}_{k}^{b}\right|=\frac{1}{2^{(k-1)(2 k-1)}}\left(\prod_{n=k+1}^{2 k-1} n !\right)^{2}\left|\operatorname{det}\left(\frac{\mathrm{B}_{a+b}}{(a+b) !}\right)_{1 \leq a, b \leq k-1}\right|
$$

and

$$
\left|\operatorname{det} \mathscr{B}_{2 k-2}^{C}\right|=\frac{1}{2^{(k-1)(2 k-3)}}\left(\prod_{n=k}^{2 k-2} n !\right)^{2}\left|\operatorname{det}\left(\frac{\mathrm{B}_{a+b}}{(a+b) !}\right)_{1 \leq a, b \leq k-1}\right|
$$

the latter of which is equal to $1 /\left|\operatorname{det} \mathbf{S}_{2 k-2}\right|>0$, by induction. Therefore, we conclude that $\mathbf{v}=\left(\mathscr{B}_{k}^{b}\right)^{-1} \mathbf{0}=$ $\mathbf{0} \in \mathbb{R}^{k-1}$. Now that $\mathbf{V}$ is filled with zeros, the skew symmetry concerning the bottom-left and the topright blocks of $\mathbf{S}_{2 k}^{-1}$ further brings us a matrix $\mathscr{B}_{k}^{b} \mathbf{U}$ filled with two columns and $(k-1)$ rows of zeros, hence an identically vanishing $\mathbf{U}$.

Thus far, we have verified $\mathbf{S}_{m}^{-1}=\mathscr{B}_{m}$ up to an even number $m=2 k$.

Inductive evaluation of $\mathbf{S}_{2 k+1} \mathscr{B}_{2 k+1}$

In view of (3.85) and (4.10), we only need to check that $\mathbf{S}_{2 k+2}^{B} \mathscr{B}_{2 k+2}^{C}=\mathbf{I}_{k+1}$.

Noting that $\left(\mathbf{S}_{2 k}^{B}\right)_{a, 0}=0$ by definition in $(1.14 \mathrm{P})$, and that $\left(\mathscr{B}_{2 k}^{C}\right)_{n, b}=\frac{4}{(2 k+2-n)(2 k+2-b)}\left(\mathscr{B}_{2 k+2}^{C}\right)_{n+1, b+1}$, we can deduce from (3.86) the following result for $a, b \in \mathbb{Z} \cap[1, k]$ :

$$
\begin{aligned}
\delta_{a, b} & =\frac{2 k+2-b}{2 k+2-a} \sum_{n=1}^{k}\left(\mathbf{S}_{\circ}^{B}\right)_{a, n}\left(\mathscr{B}_{\circ}^{C}\right)_{n, b} \\
& =\left(\mathbf{S}_{\circ 2 k+2}^{B} \mathscr{B}_{\circ}^{C}{ }_{2 k+2}\right)_{a+1, b+1}-\frac{1+(-1)^{a}}{(-1)^{\left\lfloor\frac{a}{2}\right\rfloor}} \frac{\left(\begin{array}{l}
k+1 \\
a+1
\end{array}\right)\left(\mathbf{S}_{\circ}^{B}{ }_{2 k+2}^{B} \mathscr{B}_{2 k+2}^{C}\right)_{1, b+1}}{2 k+2-a},
\end{aligned}
$$

upon invoking our induction hypothesis that $\mathbf{S}_{2 k}^{B} \mathscr{B}_{2 k}^{C}$ is the $k \times k$ identity matrix. The last displayed identity alleviates our workload to a demonstration that $\left(\mathbf{S}_{2 k+2}^{B} \mathscr{B}_{2 k+2}^{C}\right)_{1, b+1}=\left(\mathbf{S}_{2 k+2}^{B} \mathscr{B}_{\circ}^{C}{ }_{2 k+2}\right)_{b+1,1}=\delta_{b, 0}$ for all $b \in \mathbb{Z} \cap[0, k]$. This can be achieved in three steps, in the paragraphs to follow.

First, we show that $\left(\mathbf{S}_{2 k+2}^{B} \mathscr{B}_{2 k+2}^{C}\right)_{1, b+1}=0$ for $b \in \mathbb{Z} \cap[1, k]$. If $b$ is an odd number in $\mathbb{Z} \cap[1, k]$, then $\left(\mathbf{S}_{2 k+2}^{B}\right)_{1, n}\left(\mathscr{B}_{2 k+2}^{C}\right)_{n, b+1}=0$ for all $n \in \mathbb{Z} \cap[1, k+1]$, so we have $\left(\mathbf{S}_{2 k+2}^{B} \mathscr{B}_{\circ k+2}^{C}\right)_{1, b+1}=0$. If $b$ is an even number in $\mathbb{Z} \cap[2, k]$, then we have a recursion

$$
\begin{aligned}
\frac{k(k+1)\left(\mathbf{S}_{2 k+2}^{B}\right)_{1, n}\left(\mathscr{B}_{0 k+2}^{C}\right)_{n, b+1}}{(2 k+3)(2 k+2-b)}= & \frac{(b-2)\left(\mathbf{S}_{2 k}^{B}\right)_{1, n}\left(\mathscr{B}_{2 k}^{C}\right)_{n, b-1}}{2 k+2-b} \\
& +\left(1-\delta_{n, 1}\right)\left(\mathbf{S}_{\circ}^{A}\right)_{1, n-1}\left(\mathscr{B}_{\circ k}^{B}\right)_{n-1, b}+\left(\mathbf{S}_{\circ k}^{B}\right)_{1, n}\left(\mathscr{B}_{\circ k}^{D}\right)_{n, b},
\end{aligned}
$$


whose sum over $n \in \mathbb{Z} \cap[1, k+1]$ results in $\left(\mathbf{S}_{2 k+2}^{B} \mathscr{B}_{\circ k+2}^{C}\right)_{1, b+1}=0$. This is because $\left(\mathbf{S}_{2 k}^{B}\right)_{1, k+1}=0$ by definition, and our induction hypothesis on $\mathbf{S}_{2 k} \mathscr{B}_{2 k}$ leaves us an identity matrix $\mathbf{S}_{2 k}^{B} \mathscr{B}_{2 k}^{C}$ along with a $k \times k$ block $\mathbf{S}_{2 k}^{A} \mathscr{B}_{\circ k}^{B}+\mathbf{S}_{2 k}^{B} \mathscr{B}_{2 k}^{D}$ that is filled with zeros.

Second, we verify the identity $\left(\mathbf{S}_{2 k+2}^{B} \mathscr{B}_{\circ k+2}^{C}\right)_{1,1}=1$ by directly computing

$$
\left(\mathbf{S}_{2 k+2}^{B} \mathscr{B}_{02 k+2}^{C}\right)_{1,1}=\sum_{n=0}^{k}\left(\mathbf{S}_{0}^{B} B+2\right)_{1, n+1}\left(\mathscr{B}_{\circ}^{C}{ }_{2 k+2}\right)_{n+1,1}=\frac{2(-1)^{k}(2 k+3) !}{[(k+1) !]^{2}} \sum_{n=0}^{k+1} \frac{\left(\begin{array}{c}
k+1 \\
k+1-n
\end{array}\right) B_{2 k+3-n}}{2 k+3-n},
$$

and enlisting the help from a special case of the Saalschütz-Nielsen-Gelfand reciprocity relation [39, 40, 36, 27] revisited by Agoh-Dilcher [2]:

$$
(-1)^{\beta+1} \sum_{j=0}^{\alpha} \frac{\left(\begin{array}{c}
\alpha \\
j
\end{array}\right) \mathrm{B}_{\beta+1+j}}{\beta+1+j}+(-1)^{\alpha+1} \sum_{j=0}^{\beta} \frac{\left(\begin{array}{c}
\beta \\
j
\end{array}\right) \mathrm{B}_{\alpha+1+j}}{\alpha+1+j}=\frac{\alpha ! \beta !}{(\alpha+\beta+1) !},
$$

where $\alpha=\beta=k+1, j=k+1-n$.

Third, we prove that $\left(\mathbf{S}_{2 k+2}^{B} \mathscr{B}_{2 k+2}^{C}\right)_{b+1,1}=0$ for $b \in \mathbb{Z} \cap[1, k]$. In other words, we will show that in the block partition $\mathbf{S}_{2 k+2}^{B} \mathscr{B}_{0}^{C}{ }_{2 k+2}{ }^{\circ}=\left(\begin{array}{l}1 \\ \mathbf{x} \mathbf{I}_{k}\end{array}\right)$, the column vector $\mathbf{x} \in \mathbb{R}^{k}$ is in fact a zero vector. Taking determinants on both sides of the block partition, we know that the symmetric matrix $\mathbf{S}_{2}^{B}{ }_{2 k+2}$ is invertible, and its inverse $\left(\mathbf{S}_{0}^{B}{ }_{2 k+2}\right)^{-1}=\mathscr{B}_{0}^{C}{ }_{2 k+2}\left(\begin{array}{cc}1 & \mathbf{I}_{k}\end{array}\right)$ is also symmetric. Taking another block partition $\mathscr{B}_{2 k+2}^{C}=$

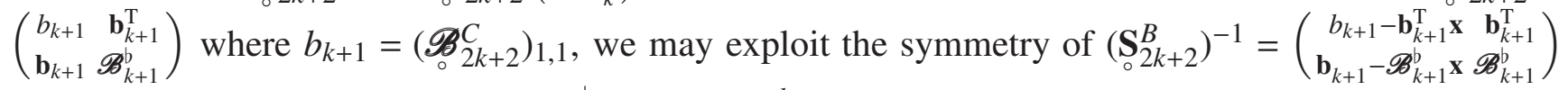
to arrive at the following relation: $\mathscr{B}_{k+1}^{b} \mathbf{x}=\mathbf{0} \in \mathbb{R}^{k}$. In view of (4.18) and (4.19), we can deduce the invertibility of $\mathscr{B}_{k+1}^{b}$ from the non-singular matrix $\mathscr{B}_{2 k}^{C}=\left(\mathbf{S}_{2 k}^{B}\right)^{-1}$, so $\mathbf{x}=\left(\mathscr{B}_{k+1}^{b}\right)^{-1} \mathbf{0}=\mathbf{0} \in \mathbb{R}^{k}$.

This completes the induction.

As a by-product of the proof above, we can quickly recover part of [25, Proposition 4.9(1)] without using any combinatorial identities concerning Bernoulli numbers.

Corollary 4.3 (Determinants of Betti matrices). The following formula holds true:

$$
\operatorname{det} \mathbf{B}_{2 k-1}=\frac{(-1)^{k-1}(2 k-1) !}{2^{5 k-1}} \Lambda_{2 k-1}
$$

When $k$ is odd, we have $\operatorname{det} \mathbf{B}_{2 k}=0$.

Proof. Taking determinants on both sides of (3.80), we arrive at $\Lambda_{2 k-1}^{2}=1 / \operatorname{det} \mathbf{S}_{2 k-1}$. Taking determinants on both sides of (4.10), we obtain $\operatorname{det} \mathbf{B}_{2 k-1} \operatorname{det} \mathbf{B}_{2 k-3} \operatorname{det} \mathbf{S}_{2 k-1}=(-1)^{k-1}(2 k+1) 4^{1-3 k}$. Hence, we can build the formula for $\operatorname{det} \mathbf{B}_{2 k-1}$ inductively on $\operatorname{det} \mathbf{B}_{1}=\frac{1}{2^{4} \cdot 3}$ and $\operatorname{det} \mathbf{B}_{2 k-1} \operatorname{det} \mathbf{B}_{2 k-3}=(-1)^{k-1}(2 k+$ 1) $4^{1-3 k} \Lambda_{2 k-1}^{2}$.

When $k$ is odd, the odd-numbered rows of $\mathbf{B}_{2 k}$ have non-zero elements in the even-numbered columns. Since there are $\left\lfloor\frac{k}{2}\right\rfloor$ such columns and $\left\lfloor\frac{k+1}{2}\right\rfloor=\left\lfloor\frac{k}{2}\right\rfloor+1$ such rows, all the rows in question must be linearly dependent. Hence $\operatorname{det} \mathbf{B}_{2 k}=0$.

For $k \in \mathbb{Z}_{>0}$, define minors $\mathbf{B}_{2 k-1}^{\mathrm{o}} \in \mathbb{Q}^{\left\lfloor\frac{k+1}{2}\right\rfloor \times\left\lfloor\frac{k+1}{2}\right\rfloor}$ and $\mathbf{B}_{2 k-1}^{\mathrm{e}} \in \mathbb{Q}^{\left\lfloor\frac{k}{2}\right\rfloor \times\left\lfloor\frac{k}{2}\right\rfloor}$ as follows:

$$
\left(\mathbf{B}_{2 k-1}^{\mathrm{o}}\right)_{a, b}=\left(\mathbf{B}_{2 k-1}\right)_{2 a-1,2 b-1}, \quad\left(\mathbf{B}_{2 k-1}^{\mathrm{e}}\right)_{a, b}=\left(\mathbf{B}_{2 k-1}\right)_{2 a, 2 b} .
$$

By convention, we set $\mathbf{B}_{1}^{\mathrm{e}}=\varnothing$ and $\operatorname{det} \mathbf{B}_{1}^{\mathrm{e}}=1$. Clearly, we have $\operatorname{det} \mathbf{B}_{2 k-1}=\operatorname{det} \mathbf{B}_{2 k-1}^{\mathrm{o}} \operatorname{det} \mathbf{B}_{2 k-1}^{\mathrm{e}}$ for all positive integers $k$. In the next corollary, we evaluate $\operatorname{det} \mathbf{B}_{2 k-1}^{\mathrm{e}}$, a result that will be used later in the proof of Proposition 4.5 .

Corollary 4.4 (Determinants of Betti minors). We have

$$
\operatorname{det} \mathbf{B}_{2 k-1}^{\mathrm{e}}=\frac{[(2 k+1) ! !]^{\frac{3-(-1)^{k}}{2}}}{(-2)^{\left\lfloor\frac{k}{2}\right\rfloor}(k-1) ! !(k ! !)^{1-(-1)^{k}}}\left[\frac{(2 k+1) ! !}{2^{k+1}}\right]^{2\left\lfloor\frac{k}{2}\right\rfloor} \prod_{j=1}^{k} \frac{(2 j)^{k-j}}{(2 j+1)^{j+1}}
$$

for each positive integer $k$. 
Proof. By rescaling and rearranging columns and rows of determinants, we have the following results for each $m \in \mathbb{Z}_{>0}$ :

$$
\begin{aligned}
\operatorname{det} \mathbf{B}_{4 m-3}^{\mathrm{o}} & =\frac{1}{2^{4 m^{2}}}\left[\prod_{n=m}^{2 m-1}(2 n) !\right]^{2} \operatorname{det}\left(\frac{\mathrm{B}_{2(a+b-1)}}{[2(a+b-1)] !}\right)_{1 \leq a, b \leq m}, \\
\operatorname{det} \mathbf{B}_{4 m-3}^{\mathrm{e}} & =\frac{1}{2^{4 m(m-1)}}\left[\prod_{n=m}^{2(m-1)}(2 n+1) !\right]^{2} \operatorname{det}\left(\frac{\mathrm{B}_{2(a+b)}}{[2(a+b)] !}\right)_{1 \leq a, b \leq m-1}, \\
\operatorname{det} \mathbf{B}_{4 m-1}^{\mathrm{o}} & =\frac{(-1)^{m}}{2^{2 m(2 m+1)}}\left[\prod_{n=m+1}^{2 m}(2 n) !\right]^{2} \operatorname{det}\left(\frac{\mathrm{B}_{2(a+b)}}{[2(a+b)] !}\right)_{1 \leq a, b \leq m}, \\
\operatorname{det} \mathbf{B}_{4 m-1}^{\mathrm{e}} & =\frac{(-1)^{m}}{2^{2 m(2 m+1)}}\left[\prod_{n=m+1}^{2 m}(2 n-1) !\right]^{2} \operatorname{det}\left(\frac{\mathrm{B}_{2(a+b-1)}}{[2(a+b-1)] !}\right)_{1 \leq a, b \leq m} .
\end{aligned}
$$

Thus, we have a recursion

$$
\begin{aligned}
-\frac{(4 m-1)(4 m-2)}{2^{5}} \frac{\Lambda_{4 m-1}}{\Lambda_{4 m-3}} & =\frac{\operatorname{det} \mathbf{B}_{4 m-1}}{\operatorname{det} \mathbf{B}_{4 m-3}}=\frac{\operatorname{det} \mathbf{B}_{4 m-1}^{\mathrm{o}} \operatorname{det} \mathbf{B}_{4 m-1}^{\mathrm{e}}}{\operatorname{det} \mathbf{B}_{4 m-3}^{\mathrm{o}} \operatorname{det} \mathbf{B}_{4 m-3}^{\mathrm{e}}} \\
& =\frac{1}{2^{8 m}}\left[\frac{(4 m) !(4 m-1) !}{(2 m) !}\right]^{2} \frac{\operatorname{det}\left(\frac{\mathrm{B}_{2(a+b)}}{[2(a+b)] !}\right)_{1 \leq a, b \leq m}}{\operatorname{det}\left(\frac{\mathrm{B}_{2(a+b)}}{[2(a+b)] !}\right)_{1 \leq a, b \leq m-1}},
\end{aligned}
$$

namely

$$
-\frac{1}{4 m+1}\left[\frac{(2 m) !}{(4 m) !}\right]^{2}=\frac{\operatorname{det}\left(\frac{\mathrm{B}_{2(a+b)}}{[2(a+b)] !}\right)_{1 \leq a, b \leq m}}{\operatorname{det}\left(\frac{\mathrm{B}_{2(a+b)}}{[2(a+b)] !}\right)_{1 \leq a, b \leq m-1}}
$$

from which we can solve

$$
\operatorname{det}\left(\frac{\mathrm{B}_{2(a+b)}}{[2(a+b)] !}\right)_{1 \leq a, b \leq m}=(-1)^{m} \prod_{n=1}^{m} \frac{[(2 n) !]^{2}}{(4 n) !(4 n+1) !} .
$$

Substituting back into (4.28) and (4.29), we may verify

$$
\begin{aligned}
\operatorname{det} \mathbf{B}_{4 m-3}^{\mathrm{e}} & =\frac{(-1)^{m+1} 2^{(5-4 m) m}}{(2 m-1) ! !(4 m-2) ! !} \prod_{n=1}^{2 m-1}(2 n-1) !, \\
\operatorname{det} \mathbf{B}_{4 m-1}^{\mathrm{o}} & =\frac{2^{-m(4 m+3)}}{(2 m) ! !(4 m+1) ! !} \prod_{n=1}^{2 m}(2 n) !,
\end{aligned}
$$

where the last equation also implies

$$
\operatorname{det} \mathbf{B}_{4 m-1}^{\mathrm{e}}=\frac{\operatorname{det} \mathbf{B}_{4 m-1}}{\operatorname{det} \mathbf{B}_{4 m-1}^{\mathrm{o}}}=\frac{(-1)^{m}}{2^{m(4 m+1)}} \frac{(2 m) ! !}{(4 m) ! !} \prod_{n=1}^{2 m}(2 n-1) !
$$

Through elementary manipulations of the factors, we may convert (4.34) and (4.36) into our statement in (4.26).

4.2. Arithmetic applications to Feynman diagrams. The Broadhurst-Roberts quadratic relations inject new insights into the arithmetic properties of on-shell Bessel moments, an important class of Feynman diagrams that respect classical equations of motion.

First, we examine period matrices $\mathbf{P}_{2 k-1}$ for the $(2 k+1)$-Bessel problems, where $k \in \mathbb{Z}_{>1}$. 
TABLE II. Some Betti matrices and de Rham matrices in Broadhurst-Roberts quadratic relations

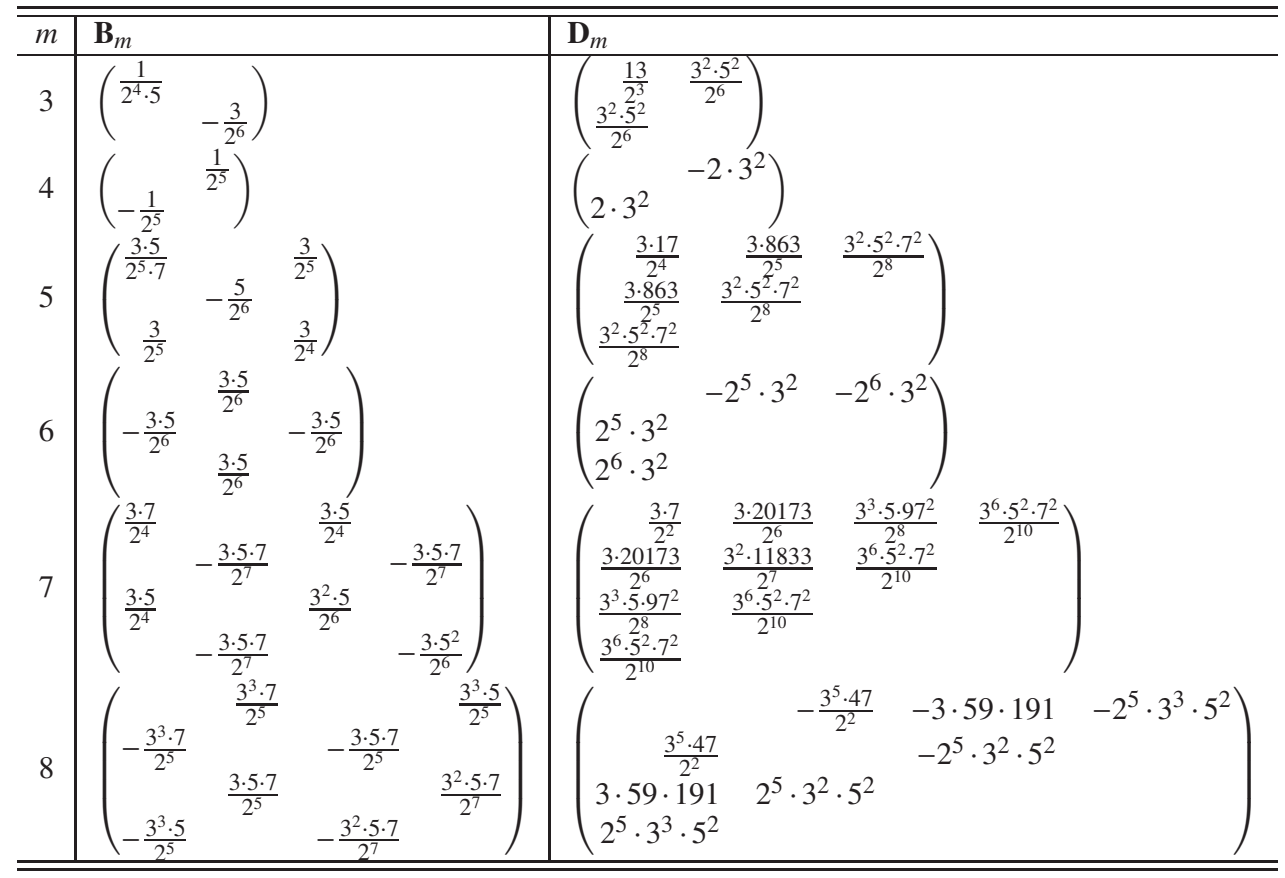

From the work of Bailey-Borwein-Broadhurst-Glasser [3], Bloch-Kerr-Vanhove [5], Samart [41], and the present author [48, 49, 54], we know that

$$
\mathbf{P}_{3}:=\left(\begin{array}{cc}
\frac{1}{\pi^{2}} \mathbf{I K M}(1,4 ; 1) & -\frac{1}{\pi^{2}} \operatorname{IKM}(1,4 ; 3) \\
\frac{1}{\pi} \operatorname{IKM}(2,3 ; 1) & -\frac{1}{\pi} \operatorname{IKM}(2,3 ; 3)
\end{array}\right)=\left(\begin{array}{rr}
C & -\left(\frac{2}{15}\right)^{2}\left(13 C-\frac{1}{10 C}\right) \\
\frac{\sqrt{15}}{2} C & -\frac{\sqrt{15}}{2}\left(\frac{2}{15}\right)^{2}\left(13 C+\frac{1}{10 C}\right)
\end{array}\right),
$$

where

$$
C:=\frac{\Gamma\left(\frac{1}{15}\right) \Gamma\left(\frac{2}{15}\right) \Gamma\left(\frac{4}{15}\right) \Gamma\left(\frac{8}{15}\right)}{240 \sqrt{5} \pi^{2}}
$$

is the "Bologna constant" attributed to Broadhurst [8, 3] and Laporta [32]. Previously, each individual entry of $\mathbf{P}_{3}=\left((-1)^{b-1} \mathcal{F}_{3, a}^{b}(1)\right)_{1 \leq a, b \leq 2}$ was computed with sophisticated arguments. Now, a quadratic relation $\mathbf{P}_{3} \mathbf{D}_{3} \mathbf{P}_{3}^{\mathrm{T}}=\mathbf{B}_{3}$ (see Table $\amalg$ for non-vanishing entries of $\mathbf{B}_{3}$ and $\mathbf{D}_{3}$ ) produces three independent quadratic equations involving Bessel moments, which immediately allow us to express all the matrix elements [cf. (4.37)] in terms of the top-left entry $\mathcal{F}_{3,1}^{1}(1)=C$.

As for the 7-Bessel problem, numerical evidence has motivated the following conjecture by Broadhurst and Mellit (see [12, (6.8)] or [9, (129)]):

$$
\operatorname{IKM}(2,5 ; 1) \stackrel{?}{=} \frac{5 \pi^{2}}{24} \zeta_{7,1}(2) .
$$

Here, we have an $L$-function associated with the 7 th symmetric power moments of Kloosterman sums

$$
\zeta_{7,1}(s):=\prod_{p} L_{p}\left(\mathbb{G}_{m, \mathbb{F}_{p}}, \mathrm{Sym}^{7} \mathrm{Kl}_{2}, s\right) \equiv \prod_{p} \frac{1}{Z_{7}\left(p, p^{-s}\right)},
$$

whose local factors at primes $p$ are written as $L_{p}\left(\mathbb{G}_{m, \mathbb{F}_{p}}, \mathrm{Sym}^{7} \mathrm{Kl}_{2}, s\right)$ in the Fu-Wan notation [26, §0] and $\frac{1}{Z_{7}\left(\frac{n}{\left.p^{-s}\right)}\right.}$ in the Broadhurst notation [9, §2]. Meanwhile, according to the Fresán-Sabbah-Yu statement [25, §7.c] of Deligne's conjecture for the motive $\mathrm{M}_{7}$ whose period realization is isomorphic to 
$\left(\mathrm{H}_{\mathrm{dR}, \text { mid }}^{1}\left(\mathbb{G}_{m}, \mathrm{Sym}^{7} \mathrm{Kl}_{2}\right), \mathrm{H}_{1}^{\mathrm{mid}}\left(\mathbb{G}_{m}, \mathrm{Sym}^{7} \mathrm{Kl}_{2}\right), \mathrm{P}_{7}^{\text {mid }}\right)$, one has

$$
\operatorname{det}\left(\begin{array}{ll}
\operatorname{IKM}(1,6 ; 1) & \operatorname{IKM}(1,6 ; 3) \\
\mathbf{I K M}(3,4 ; 1) & \operatorname{IKM}(3,4 ; 3)
\end{array}\right) \stackrel{?}{=} \frac{\pi^{4}}{14 \sqrt{105}} \zeta_{7,1}(2) .
$$

In transcribing the conjectural identity above, we have already exploited a relation between $\zeta_{7,1}(2)$ and $\zeta_{7,1}(3)$, which results from a functional equation conjectured by Broadhurst-Mellit (see [12, (6.7)] or [9, (128)]) and verified by Fresán-Sabbah-Yu [23, Theorem 1.2]:

$$
\Lambda_{7}(s):=\left(\frac{105}{\pi^{3}}\right)^{s / 2} \Gamma\left(\frac{s-1}{2}\right) \Gamma\left(\frac{s}{2}\right) \Gamma\left(\frac{s+1}{2}\right) \zeta_{7,1}(s)=\Lambda_{7}(5-s) .
$$

The conjectures (4.39) and (4.41) are actually equivalent to each other, since we have

$$
\mathcal{F}_{5,2}^{1}(1)=-\frac{1}{2^{2}} \sqrt{\frac{5^{3} \cdot 7^{3}}{3}} \operatorname{det}\left(\begin{array}{cc}
\mathcal{F}_{5,1}^{1}(1) & \mathcal{F}_{5,1}^{2}(1) \\
\mathcal{F}_{5,3}^{1}(1) & \mathcal{F}_{5,3}^{2}(1)
\end{array}\right)
$$

by reading off the element in the second row and the first column from both sides of $\mathbf{P}_{5}=\mathbf{B}_{5} \frac{\operatorname{cof} \mathbf{P}_{5}}{\operatorname{det} \mathbf{P}_{5}} \mathbf{D}_{5}^{-1}$, which is a reformulation of the quadratic relation $\mathbf{P}_{5} \mathbf{D}_{5} \mathbf{P}_{5}^{\mathrm{T}}=\mathbf{B}_{5}$ using the determinant $\operatorname{det} \mathbf{P}_{5}=-\frac{2^{4}}{\sqrt{3^{3} 5^{5} 7^{7}}}$ and the cofactor matrix $\operatorname{cof} \mathbf{P}_{5}=\left(\operatorname{det} \mathbf{P}_{5}\right)\left(\mathbf{P}_{5}^{\mathrm{T}}\right)^{-1}$. The sum rule in (4.43) is in fact a continuation of a pattern attested in $\mathbf{P}_{3}$ [see (4.37)]:

$$
\mathcal{F}_{3,2}^{1}(1)=\frac{\sqrt{3 \cdot 5}}{2} \mathcal{F}_{3,1}^{1}(1),
$$

which in turn, is compatible with proven instances [38, 41] of Deligne's conjecture for the motive $\mathrm{M}_{5}$ :

$$
\mathcal{F}_{3,1}^{1}(1)=\frac{1}{5} \zeta_{5,1}(1), \quad \mathcal{F}_{3,2}^{1}(1)=\frac{3}{4 \pi} \zeta_{5,1}(2) .
$$

Here,

$$
\zeta_{5,1}(s):=\prod_{p} L_{p}\left(\mathbb{G}_{m, \mathbb{F}_{p}}, \operatorname{Sym}^{5} \mathrm{Kl}_{2}, s\right) \equiv \prod_{p} \frac{1}{Z_{5}\left(p, p^{-s}\right)}=\int_{0}^{\infty} \frac{(2 \pi y)^{s}}{\Gamma(s)} \frac{f_{3,15}(i y) \mathrm{d} y}{y}
$$

is the $L$-function associated with the cusp form $f_{3,15}(z):=[\eta(3 z) \eta(5 z)]^{3}+[\eta(z) \eta(15 z)]^{3}$ of weight 3 and level 15 [37], satisfying a functional equation [9, (95)]:

$$
\Lambda_{5}(s):=\left(\frac{15}{\pi^{2}}\right)^{s / 2} \Gamma\left(\frac{s}{2}\right) \Gamma\left(\frac{s+1}{2}\right) \zeta_{5,1}(s)=\Lambda_{5}(3-s) .
$$

Trading each element in a minor determinant of $\mathbf{P}_{7}$ for its counterpart in $\mathbf{B}_{7} \frac{\operatorname{cof} \mathbf{P}_{7}}{\operatorname{det} \mathbf{P}_{7}} \mathbf{D}_{7}^{-1}$, we can also construct another sum rule

$$
\operatorname{det}\left(\begin{array}{cc}
\mathcal{F}_{7,2}^{1}(1) & \mathcal{F}_{7,2}^{2}(1) \\
\mathcal{F}_{7,4}^{1}(1) & \mathcal{F}_{7,4}^{2}(1)
\end{array}\right)=\frac{\sqrt{3 \cdot 5 \cdot 7}}{2^{2}} \operatorname{det}\left(\begin{array}{cc}
\mathcal{F}_{7,1}^{1}(1) & \mathcal{F}_{7,1}^{2}(1) \\
\mathcal{F}_{7,3}^{1}(1) & \mathcal{F}_{7,3}^{2}(1)
\end{array}\right),
$$

which is compatible with Deligne's conjecture [25, §7.c] for the motive $\mathrm{M}_{9}$ and the corresponding functional equation [23, Theorem 1.2] for $\zeta_{9,1}(s)$. For an arbitrarily large $k$, there is a similar identity connecting two minor determinants of $\mathbf{P}_{2 k-1}$, as established in the proposition below.

Proposition 4.5 (Reflection formula for minor determinants of $\mathbf{P}_{2 k-1}$ ). For $k \in \mathbb{Z}_{>1}$, we have an identity that is equivalent to 1.21):

$$
\begin{aligned}
& \operatorname{det}\left((-1)^{b-1} \mathcal{F}_{2 k-1,2 a}^{b}(1)\right)_{1 \leq a, b \leq\left\lfloor\frac{k}{2}\right\rfloor} \\
= & (-1)^{\left\lfloor\frac{k+1}{4}\right\rfloor+\left\lfloor\frac{1}{2}\left\lfloor\frac{k}{2}\right\rfloor\right\rfloor} \frac{\sqrt{[(2 k+1) ! !]^{2-(-1)^{k}}}}{2^{\left\lfloor\frac{k}{2}\right\rfloor(k-1) ! !(k ! !)^{1-(-1)^{k}}}} \operatorname{det}\left((-1)^{b-1} \mathcal{F}_{2 k-1,2 a-1}^{b}(1)\right)_{1 \leq a, b \leq\left\lfloor\frac{k+1}{2}\right\rfloor} .
\end{aligned}
$$


Here, the leading factor on the right-hand side can be rewritten as

$$
(-1)^{\left\lfloor\frac{k+1}{4}\right\rfloor+\left\lfloor\frac{1}{2}\left\lfloor\frac{k}{2}\right\rfloor\right\rfloor}=\frac{\operatorname{det}\left((-1)^{b-1} \delta_{a, b}\right)_{1 \leq a, b \leq\left\lfloor\frac{k}{2}\right\rfloor}}{\operatorname{det}\left((-1)^{b-1} \delta_{a, b}\right)_{1 \leq a, b \leq\left\lfloor\frac{k+1}{2}\right\rfloor}}= \begin{cases}-1, & k \equiv 3 \quad(\bmod 4), \\ 1, & \text { otherwise. }\end{cases}
$$

Proof. Suppose that we partition an invertible matrix into four blocks $\mathbf{M}=\left(\begin{array}{c}\mathbf{C} \\ \mathbf{D}\end{array}\right)$, where the top-left block $\mathbf{A}$ is also invertible. The Banachiewicz formula [4] provides us with a partition

$$
\begin{aligned}
\mathbf{M}^{-1} & =\left(\begin{array}{rr}
\mathbf{I} & -\mathbf{A}^{-1} \mathbf{B} \\
\mathbf{I}
\end{array}\right)\left(\begin{array}{lr}
\mathbf{A}^{-1} & \left(\mathbf{D}-\mathbf{C A}^{-1} \mathbf{B}\right)^{-1}
\end{array}\right)\left(\begin{array}{ll}
\mathbf{I} & \\
-\mathbf{C A}^{-1} & \mathbf{I}
\end{array}\right) \\
& =\left(\begin{array}{rr}
\mathbf{A}^{-1}+\mathbf{A}^{-1} \mathbf{B}\left(\mathbf{D}-\mathbf{C A}^{-1} \mathbf{B}\right)^{-1} \mathbf{C A}^{-1} & -\mathbf{A}^{-1} \mathbf{B}\left(\mathbf{D}-\mathbf{C A}^{-1} \mathbf{B}\right)^{-1} \\
-\left(\mathbf{D}-\mathbf{C A}^{-1} \mathbf{B}\right)^{-1} \mathbf{C A}^{-1} & \left(\mathbf{D}-\mathbf{C A}^{-1} \mathbf{B}\right)^{-1}
\end{array}\right),
\end{aligned}
$$

where I stands for an identity matrix of appropriate size. Taking determinants on the first equality in (4.51), we have $\operatorname{det}(\mathbf{M})=\operatorname{det}(\mathbf{A}) \operatorname{det}\left(\mathbf{D}-\mathbf{C A}^{-1} \mathbf{B}\right)$.

Define

$$
\widehat{\mathbf{P}}_{2 k-1}=\left(\begin{array}{l}
\left((-1)^{b-1} \mathcal{F}_{2 k-1,2 a-1}^{b}(1)\right)_{1 \leq a \leq\left\lfloor\frac{k+1}{2}\right\rfloor, 1 \leq b \leq k} \\
\left((-1)^{b-1} \mathcal{F}_{2 k-1,2 a}^{b}(1)\right)_{1 \leq a \leq\left\lfloor\frac{k}{2}\right\rfloor, 1 \leq b \leq k}
\end{array}\right)
$$

by reshuffling rows of $\mathbf{P}_{2 k-1}$. The Broadhurst-Roberts quadratic relation can be reformulated into

$$
\widehat{\mathbf{P}}_{2 k-1} \mathbf{D}_{2 k-1}=\left(\begin{array}{ll}
\mathbf{B}_{2 k-1}^{\mathrm{o}} & \\
& \mathbf{B}_{2 k-1}^{\mathrm{e}}
\end{array}\right)\left(\widehat{\mathbf{P}}_{2 k-1}^{\mathrm{T}}\right)^{-1},
$$

where $\mathbf{B}_{2 k-1}^{\mathrm{o}}$ and $\mathbf{B}_{2 k-1}^{\mathrm{e}}$ are defined in (4.25).

Suppose that $\operatorname{det}\left((-1)^{b-1} \mathcal{F}_{2 k-1,2 a-1}^{b}(1)\right)_{1 \leq a, b \leq\left\lfloor\frac{k+1}{2}\right\rfloor} \neq 0$, and take a matrix partition

$$
\widehat{\mathbf{P}}_{2 k-1}=\left(\begin{array}{ll}
\widehat{\mathbf{A}}:=\left((-1)^{b-1} \mathcal{F}_{2 k-1,2 a-1}^{b}(1)\right)_{1 \leq a, b \leq\left\lfloor\frac{k+1}{2}\right\rfloor} & \widehat{\mathbf{B}} \\
\widehat{\mathbf{C}} & \widehat{\mathbf{D}}
\end{array}\right) .
$$

We may read off the bottom-right $\left\lfloor\frac{k}{2}\right\rfloor \times\left\lfloor\frac{k}{2}\right\rfloor$ block from both sides of (4.53), while referring back to the Banachiewicz formula (4.51), as follows:

$$
\left((-1)^{b-1} \mathcal{F}_{2 k-1,2 a}^{b}(1)\right)_{1 \leq a, b \leq\left\lfloor\frac{k}{2}\right\rfloor} \mathbf{D}_{2 k-1}^{\nabla}=\mathbf{B}_{2 k-1}^{\mathrm{e}}\left[\widehat{\mathbf{D}}^{\mathrm{T}}-\widehat{\mathbf{B}}^{\mathrm{T}}\left(\widehat{\mathbf{A}}^{-1}\right)^{\mathrm{T}} \widehat{\mathbf{C}}^{\mathrm{T}}\right]^{-1} .
$$

Here, no matter $k$ is even or odd, the matrix $\mathbf{D}_{2 k-1}^{\nabla}$ is extracted from the top-right $\left\lfloor\frac{k}{2}\right\rfloor \times\left\lfloor\frac{k}{2}\right\rfloor$ block of $\mathbf{D}_{2 k-1}$. We may quote in advance from Corollary 4.7 that $\mathbf{D}_{2 k-1}^{\nabla}$ is upper-left triangular, with all its anti-diagonal elements being $\left[\frac{(2 k+1) ! !}{2^{k+1}}\right]^{2}$. Since

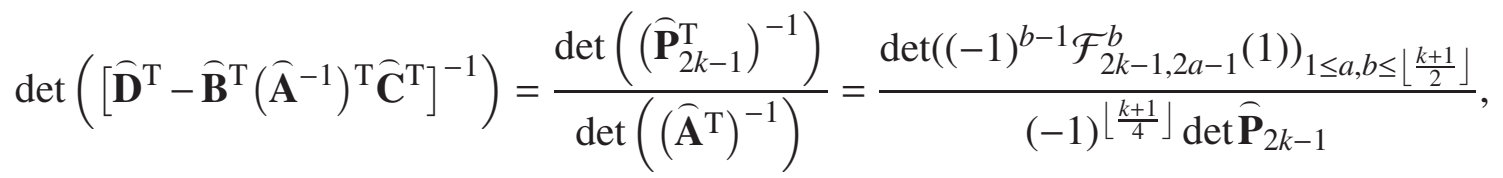

we can deduce

$$
\operatorname{det}\left((-1)^{b-1} \mathcal{F}_{2 k-1,2 a}^{b}(1)\right)_{1 \leq a, b \leq\left\lfloor\frac{k}{2}\right\rfloor}=\frac{(-1)^{\left\lfloor\frac{k+1}{4}\right\rfloor} \operatorname{det} \mathbf{B}_{2 k-1}^{\mathrm{e}}}{\operatorname{det} \widehat{\mathbf{P}}_{2 k-1} \operatorname{det} \mathbf{D}_{2 k-1}^{\nabla}} \operatorname{det}\left((-1)^{b-1} \mathcal{F}_{2 k-1,2 a-1}^{b}(1)\right)_{1 \leq a, b \leq\left\lfloor\frac{k+1}{2}\right\rfloor}
$$

from (4.55). Simplifying the right-hand side of the equation above with $\operatorname{det} \widehat{\mathbf{P}}_{2 k-1}=(-1)^{\left\lfloor\frac{k}{2}\right\rfloor} \prod_{j=1}^{k} \frac{(2 j)^{k-j}}{\sqrt{(2 j+1)^{2 j+1}}}$,

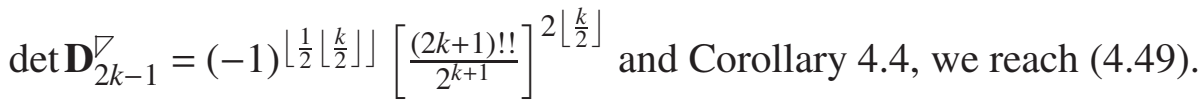


Starting from an alternative assumption that $\operatorname{det}\left((-1)^{b-1} \mathcal{F}_{2 k-1,2 a}^{b}(1)\right)_{1 \leq a, b \leq\left\lfloor\frac{k}{2}\right\rfloor} \neq 0$, while switching the rôles of $\left((-1)^{b-1} \mathcal{F}_{2 k-1,2 a-1}^{b}(1)\right)_{1 \leq a \leq\left\lfloor\frac{k+1}{2}\right\rfloor, 1 \leq b \leq k}$ and $\left((-1)^{b-1} \mathcal{F}_{2 k-1,2 a}^{b}(1)\right)_{1 \leq a \leq\left\lfloor\frac{k}{2}\right\rfloor, 1 \leq b \leq k}$, one can repeat the procedures above to arrive at the same identity in (4.49).

If both $\operatorname{det}\left((-1)^{b-1} \mathcal{F}_{2 k-1,2 a-1}^{b}(1)\right)_{1 \leq a, b \leq\left\lfloor\frac{k+1}{2}\right\rfloor}$ and $\operatorname{det}\left((-1)^{b-1} \mathcal{F}_{2 k-1,2 a}^{b}(1)\right)_{1 \leq a, b \leq\left\lfloor\frac{k}{2}\right\rfloor}$ vanish, then (4.49) is trivially true.

Remark The reflection formula (4.49) applies retroactively to the case where $k=1$, so long as we adopt the convention that $\operatorname{det} \varnothing=1$. In this case, the classical evaluation $\mathbf{I K M}(1,2 ; 1)=\frac{\pi}{3 \sqrt{3}}[3,(23)]$ is recovered.

The cases of $\mathbf{P}_{2 k}, k \in \mathbb{Z}_{>1}$ involve additional subtleties.

The quadratic relation $\mathbf{P}_{4} \mathbf{D}_{4} \mathbf{P}_{4}^{\mathrm{T}}=\mathbf{B}_{4}$ (see Table $\amalg$ for non-vanishing entries of $\mathbf{B}_{4}$ and $\mathbf{D}_{4}$ ) produces only one independent quadratic equation for Bessel moments, which is equivalent to $\operatorname{det} \mathbf{P}_{4}=-\frac{1}{2^{6} 3^{2}}$. Thus, we do not have news for the 6-Bessel problem. Especially, on the algebraic side, there seem to be no quadratic mechanisms to relate the ratio $\left[\mathcal{F}_{4,2}^{1}(1)\right]^{2} /\left[\mathcal{F}_{4,1}^{1}(1)\right]^{2}$ to a rational number; on the analytic side, the proven instances [48, §4] of Deligne's conjecture for the motive $\mathrm{M}_{6}$ bring us two evaluations $\pi^{5 / 2} \mathcal{F}_{4,1}^{1}(1)=\frac{\pi^{2}}{2} \zeta_{6,1}(2)$ and $\pi^{3 / 2} \mathcal{F}_{4,2}^{1}(1)=\frac{\pi^{2}}{2} \zeta_{6,1}(1)=\frac{3}{2} \zeta_{6,1}(3)$, which are not connected to each other by the functional equation [9, (106)]

$$
\Lambda_{6}(s):=\left(\frac{6}{\pi^{2}}\right)^{s / 2} \Gamma\left(\frac{s}{2}\right) \Gamma\left(\frac{s+1}{2}\right) \zeta_{6,1}(s)=\Lambda_{6}(4-s)
$$

for $\zeta_{6,1}(s)$, the $L$-function associated with the cusp form $f_{4,6}(z):=[\eta(z) \eta(2 z) \eta(3 z) \eta(6 z)]^{2}$ of weight 4 and level 6 [30].

Unlike the situations discussed so far, the $L$-function for the motive $\mathrm{M}_{8}$ needs an additional correction factor, in the form of $[9, \S 7.6]$

$$
L\left(f_{6,6}, s\right):=\int_{0}^{\infty} \frac{(2 \pi y)^{s}}{\Gamma(s)} \frac{f_{6,6}(i y) \mathrm{d} y}{y}=\prod_{p} \frac{Z_{4}\left(p, p^{4-s}\right)}{Z_{8}\left(p, p^{-s}\right)},
$$

where the associated cusp form [47]

$$
f_{6,6}(z):=\frac{[\eta(2 z) \eta(3 z)]^{9}}{[\eta(z) \eta(6 z)]^{3}}+\frac{[\eta(z) \eta(6 z)]^{9}}{[\eta(2 z) \eta(3 z)]^{3}}
$$

has weight 6 and level 6. The Fresán-Sabbah-Yu statement [25, §7.c] of Deligne's conjecture holds for the motive $\mathrm{M}_{8}$, according to the following identities conjectured by Broadhurst [9, §7.6] and proved by the present author (see [48, §5] as well as [54, §3.1]): $\pi^{7 / 2} \mathcal{F}_{6,1}^{1}(1)=\pi^{7 / 2} \mathcal{F}_{6,3}^{1}(1)=\frac{\pi^{4}}{4} L\left(f_{6,6}, 2\right)$, $\pi^{5 / 2} \mathcal{F}_{6,2}^{1}(1)=\frac{\pi^{4}}{8} L\left(f_{6,6}, 1\right)=\frac{9 \pi^{2}}{14} L\left(f_{6,6}, 3\right)$. The quadratic relation $\mathbf{P}_{6} \mathbf{D}_{6} \mathbf{P}_{6}^{\mathrm{T}}=\mathbf{B}_{6}$ produces three independent quadratic equations concerning on-shell Bessel moments, which in turn, can be reduced (say, by computation of Gröbner basis) to the following sum rules:

$$
\begin{aligned}
\mathcal{F}_{6,1}^{1}(1) & =\mathcal{F}_{6,3}^{1}(1), \\
\operatorname{det}\left(\begin{array}{cc}
\mathcal{F}_{6,2}^{1}(1) & \mathcal{F}_{6,2}^{2}(1)+2 \mathcal{F}_{6,2}^{3}(1) \\
\mathcal{F}_{6,3}^{1}(1) & \mathcal{F}_{6,3}^{2}(1)+2 \mathcal{F}_{6,3}^{3}(1)
\end{array}\right) & =\frac{5}{2^{11 \cdot 3}} .
\end{aligned}
$$

All these three identities have been reported earlier [53, (98)]. Moreover, we point out that the quadratic relation $\mathbf{P}_{6} \mathbf{D}_{6} \mathbf{P}_{6}^{\mathrm{T}}=\mathbf{B}_{6}$ does not exhaust algebraic relations $\left[\right.$ over $\mathbb{Q}(\pi)$ ] for the entries of $\mathbf{P}_{6}$. Noticeably, such a relation does not entail the following identities [54, Theorem 1.3]:

$$
\mathcal{F}_{6,1}^{1}(1)+2^{3} \cdot 3^{2} \mathcal{F}_{6,1}^{2}(1)=\frac{7 \sqrt{\pi}}{2^{4} \cdot 3}
$$




$$
\begin{gathered}
\mathcal{F}_{6,2}^{1}(1)+2^{3} \cdot 3^{2} \mathcal{F}_{6,2}^{2}(1)=0, \\
\mathcal{F}_{6,3}^{1}(1)+2^{3} \cdot 3^{2} \mathcal{F}_{6,3}^{2}(1)=-\frac{5 \sqrt{\pi}}{2^{2} \cdot 3}, \\
2^{8} \cdot 3^{3} \operatorname{det}\left(\begin{array}{cc}
\mathcal{F}_{6,1}^{1}(1) & \mathcal{F}_{6,1}^{3}(1) \\
\mathcal{F}_{6,2}^{1}(1) & \mathcal{F}_{6,2}^{3}(1)
\end{array}\right)+\frac{3^{2} \cdot 5}{2^{4}}=7 \sqrt{\pi} \mathcal{F}_{6,2}^{1}(1),
\end{gathered}
$$

which are all provable by analytic properties of the cusp form $f_{6,6}(z)[54, \S 3]$.

We say that a sum rule $P\left(\mathcal{F}_{8,1}^{1}(1), \mathcal{F}_{8,1}^{2}(1), \ldots, \mathcal{F}_{8,4}^{4}(1)\right)=0$ for the 10 -Bessel problem arises from a "trivial factor" if $P\left(x_{1,1}, x_{1,2}, \ldots, x_{4,4}\right) \in \mathbb{Z}\left[x_{1,1}, x_{1,2}, \ldots, x_{4,4}\right]$ divides the following polynomial in 16 formal variables belonging to the matrix $\mathbf{X}_{4}=\left(x_{a, b}\right)_{1 \leq a, b \leq 4}$ :

$$
\prod_{1 \leq a<b \leq 4}\left(\mathbf{X}_{4} \mathbf{D}_{8} \mathbf{X}_{4}^{\mathrm{T}}-\mathbf{B}_{8}\right)_{a, b} \prod_{m=1}^{4} \prod_{n=1}^{4}\left(\mathbf{B}_{8}^{-1} \mathbf{X}_{4} \mathbf{D}_{8}-\frac{\operatorname{cof} \mathbf{X}_{4}}{\operatorname{det} \mathbf{P}_{8}}\right)_{m, n} .
$$

After constructing a Gröbner basis that generates the ideal

$$
\left\langle\mathbf{X}_{4} \mathbf{D}_{8} \mathbf{X}_{4}^{\mathrm{T}}-\mathbf{B}_{8}\right\rangle:=\left\{\sum_{1 \leq a<b \leq 4}\left(\mathbf{X}_{4} \mathbf{D}_{8} \mathbf{X}_{4}^{\mathrm{T}}-\mathbf{B}_{8}\right)_{a, b} p_{a, b} \mid p_{a, b} \in \mathbb{C}\left[x_{1,1}, x_{1,2}, \ldots, x_{4,4}\right]\right\},
$$

one can find 6 sum rules (loci of polynomials in the aforementioned ideal) that do not arise from "trivial factors":

$$
\begin{aligned}
& 5 \cdot 7 \operatorname{det}\left(\begin{array}{ll}
\mathcal{F}_{8,1}^{b}(1) & 3 \mathcal{F}_{8,2}^{b}(1)-2^{2} \mathcal{F}_{8,4}^{b}(1) \\
\mathcal{F}_{8,1}^{b^{\prime}}(1) & 3 \mathcal{F}_{8,2}^{b^{\prime}}(1)-2^{2} \mathcal{F}_{8,4}^{b^{\prime}}(1)
\end{array}\right)-2^{2} \cdot 3^{2} \operatorname{det}\left(\begin{array}{lll}
\mathcal{F}_{8,3}^{b}(1) & 5 \mathcal{F}_{8,2}^{b}(1)-7 \mathcal{F}_{8,4}^{b}(1) \\
\mathcal{F}_{8,3}^{b^{\prime}}(1) & 5 \mathcal{F}_{8,2}^{b^{\prime}}(1)-7 \mathcal{F}_{8,4}^{b^{\prime}}(1)
\end{array}\right) \\
&= \begin{cases}0, & b=1, b^{\prime} \in\{2,3\}, \\
-\frac{7}{2^{10.5}}, & b=1, b^{\prime}=4 \\
-\frac{3 \cdot 7}{2^{10.5}}, & b=2, b^{\prime}=3 \\
\frac{7.59 \cdot 191}{2^{15} \cdot 3 \cdot 5^{3}}, & b=2, b^{\prime}=4 \\
-\frac{3 \cdot 7 \cdot 47}{2^{17} \cdot 5^{3}}, & b=3, b^{\prime}=4 .\end{cases}
\end{aligned}
$$

None of them resembles the situation (4.48) in $\mathbf{P}_{7}$. If one rewrites $\operatorname{det}\left(\begin{array}{cc}\mathcal{F}_{8,2}^{1}(1) & \mathcal{F}_{8,2}^{2}(1) \\ \mathcal{F}_{8,4}^{1}(1) & \mathcal{F}_{8,4}^{2}(1)\end{array}\right)\left[\operatorname{ordet}\left(\begin{array}{ll}\mathcal{F}_{8,1}^{1}(1) & \mathcal{F}_{8,1}^{2}(1) \\ \mathcal{F}_{8,3}^{1}(1) & \mathcal{F}_{8,3}^{2}(1)\end{array}\right)\right]$ by replacing every entry of $\mathbf{P}_{8}$ with its counterpart in $\mathbf{B}_{8} \frac{\operatorname{cof} \mathbf{P}_{8}}{\operatorname{det} \mathbf{P}_{8}} \mathbf{D}_{8}^{-1}$, then one just gets back the determinant itself, instead of a different minor. Analytically, this is understandable, due to the lack of a functional connection between critical $L$-values that are (conjecturally) $\mathbb{Q}^{\times} \sqrt{\pi}^{\mathbb{Z}}$ multiples of the aforementioned minor determinants. Algebraically, the gadget in our proof of Proposition 4.5 no longer works here, because the row-reshuffled matrix $\widehat{\mathbf{P}}_{8}$ satisfies

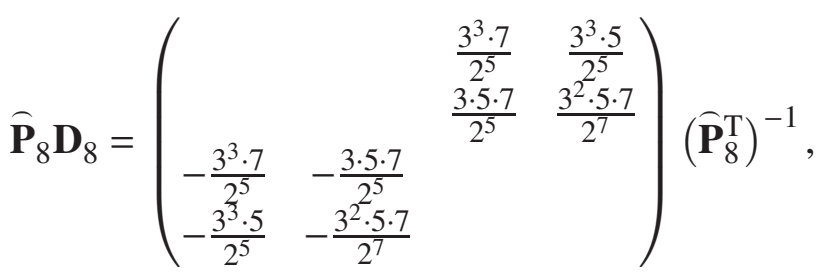

where the row- and column-reshuffled Betti matrix no longer has block diagonal form-the bottom-left (as opposed to bottom-right) block of the Banachiewicz formula (4.51) results in a tautological statement

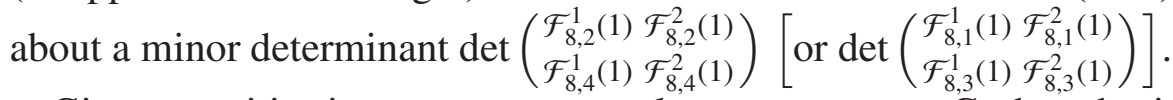

Given a positive integer $m$, one can always construct a Gröbner basis for the Broadhurst-Roberts ideal $I_{m+2}^{\mathrm{BR}}=\left\langle\mathbf{X}_{\lfloor(m+1) / 2\rfloor} \mathbf{D}_{m} \mathbf{X}_{\lfloor(m+1) / 2\rfloor}^{\mathrm{T}}-\mathbf{B}_{m}\right\rangle$, using the Buchberger algorithm [16]. For moderately large $m$, 
preliminary experiments in this direction have not yet produced any new $\mathbb{Q}$-linear sum rules [like (4.65)] that go beyond the relations for generalized Crandall numbers (2.47).

Broadhurst and Roberts originally speculated that the quadratic equation $\mathbf{P}_{m} \mathbf{D}_{m} \mathbf{P}_{m}^{\mathrm{T}}=\mathbf{B}_{m}$ delivers a complete set of algebraic relations among entries of $\mathbf{P}_{m}$, unless $(m-2)$ is an integer multiple of 4. Fresán, Sabbah and Yu provided heuristic justification (see comments after [25, Theorem 1.4]) of this speculation, citing Grothendieck's period conjecture that equates the transcendental degree of $\mathbb{Q}\left(\mathbf{P}_{m}\right)$ with the dimension of the motivic Galois group for $\mathbf{P}_{m}$. The aberrant sum rule (4.65) serves as a reminder that there may exist very surprising algebraic relations when $m \equiv 2(\bmod 4)$.

In view of the foregoing applications, we can say that the Broadhurst-Roberts quadratic relations convey more detailed messages about on-shell Bessel moments than the Broadhurst-Mellit determinant formulae. Perhaps paradoxically, the algebraic mechanism underlying the former relations is essentially identical to what we have explored and exploited during the proof [49] of the latter, namely Wrońskian algebra for homogeneous solutions to Vanhove's differential equations.

Our off-shell generalizations (Theorem 1.1) to the Broadhurst-Roberts quadratic relations also have some interesting consequences.

If we define $\dot{\mathcal{F}}_{m, j}^{\ell}(u):=\sqrt{u} \dot{\mathcal{F}}_{m, j}^{\ell}(u)=2 u D^{1} \mathcal{F}_{m, j}^{\ell}(u)$, then the Gröbner basis of the off-shell ideal $\left\langle\mathcal{L}_{4}(u) \mathbf{W}_{4}(u) \mathbf{S}_{4} \mathbf{W}_{4}^{\mathrm{T}}(u) \mathbf{V}_{4}(u)+\mathbf{I}_{4}\right\rangle$ offers us a relation

$$
\begin{aligned}
& 0=2 u\left(3 u^{2}-70 u+259\right) \operatorname{det}\left(\begin{array}{cc}
\mathcal{F}_{4,2}^{1}(u) & \mathcal{F}_{4,3}^{1}(u) \\
\mathcal{F}_{4,2}^{2}(u) & \mathcal{F}_{4,3}^{2}(u)
\end{array}\right)+8(u-13)(u-1) \operatorname{det}\left(\begin{array}{cc}
\mathcal{F}_{4,2}^{1}(u) & \mathcal{F}_{4,3}^{1}(u) \\
\dot{\mathcal{F}}_{4,2}^{1}(u) & \dot{\mathcal{F}}_{4,3}^{1}(u)
\end{array}\right) \\
& +(u-25)(u-9)(u-1)\left[\operatorname{det}\left(\begin{array}{cc}
\mathcal{F}_{4,2}^{1}(u) & \mathcal{F}_{4,3}^{1}(u) \\
\dot{\mathcal{F}}_{4,2}^{2}(u) & \dot{\mathcal{F}}_{4,3}^{2}(u)
\end{array}\right)+\operatorname{det}\left(\begin{array}{cc}
\mathcal{F}_{4,2}^{2}(u) & \mathcal{F}_{4,3}^{2}(u) \\
\dot{\mathcal{F}}_{4,2}^{1}(u) & \dot{\mathcal{F}}_{4,3}^{1}(u)
\end{array}\right)\right]
\end{aligned}
$$

for $u \in(0,1)$, which extends to $u \in(-\infty, 1)$ by analytic continuation. The last displayed identity reveals equivalence between two conjectural determinant formulae of Broadhurst (see [11, §3] or [1, §5.3])

$$
\begin{array}{r}
\operatorname{det}\left(\begin{array}{ll}
3 \mathcal{F}_{4,3}^{1}(-7)+4 \dot{\mathcal{F}}_{4,3}^{1}(-7) & \mathcal{F}_{4,3}^{1}(-7)+28 \mathcal{F}_{4,3}^{2}(-7) \\
3 \mathcal{F}_{4,2}^{1}(-7)+4 \dot{\mathcal{F}}_{4,2}^{1}(-7) & \mathcal{F}_{4,2}^{1}(-7)+28 \mathcal{F}_{4,2}^{2}(-7)
\end{array}\right) \stackrel{?}{=}-\frac{3}{32}, \\
\operatorname{det}\left(\begin{array}{ll}
\mathcal{F}_{4,3}^{1}(-7) & 39 \dot{\mathcal{F}}_{4,3}^{1}(-7)-427 \mathcal{F}_{4,3}^{2}(-7)-112 \dot{\mathcal{F}}_{4,3}^{2}(-7) \\
\mathcal{F}_{4,2}^{1}(-7) & 39 \dot{\mathcal{F}}_{4,2}^{1}(-7)-427 \mathcal{F}_{4,2}^{2}(-7)-112 \dot{\mathcal{F}}_{4,2}^{2}(-7)
\end{array}\right) \stackrel{?}{=} \frac{3}{32},
\end{array}
$$

which in turn, are inspired by the recent work of Candelas, de la Ossa, Elmi, and van Straten [18]. Similarly, for $u=(\sqrt{17}-4)^{2}$, one can demonstrate that two determinant formulae conjectured by Broadhurst (see [11, §4] or [1, §5.4]) are equivalent to each other.

It is perhaps appropriate to ask whether there is an algebro-geometric interpretation of our off-shell quadratic relations, in the spirit of Fresán-Jossen [22] and Fresán-Sabbah-Yu [23, 24, 25]. We would like to see such an extension of the motivic method to certain algebraic values of the off-shell parameter $u$. In the special cases of $\mathcal{F}_{4,2}^{1}(-7), \mathcal{F}_{4,3}^{1}(-7), \mathcal{F}_{4,2}^{1}\left((\sqrt{17}-4)^{2}\right)$ and $\mathcal{F}_{4,3}^{1}\left((\sqrt{17}-4)^{2}\right)$, we hope that powerful tools in algebraic geometry may eventually lead to proofs of Broadhurst's recent conjectures (see [11, $\S \S 3-4]$ or [1, §§5.3-5.4]) that evaluate these off-shell Bessel moments in terms of special $L$-values.

4.3. Alternative representations of de Rham matrices. From the invertibility of $\mathbf{P}_{m}$ guaranteed by (1.2), we know that the de Rham matrix $\mathbf{D}_{m}$ satisfying the Broadhurst-Roberts quadratic relation $\mathbf{P}_{m} \mathbf{D}_{m} \mathbf{P}_{m}^{\mathrm{T}}=$ $\mathbf{B}_{m}$ is unique. Nonetheless, there exist several different expressions for these de Rham matrices, whose mutual equivalence is not self-evident (absent the uniqueness argument).

Proposition 4.6 (Representations of $\left.\mathbf{D}_{m}\right)$. Let $\boldsymbol{\beta}_{m}(u)$ be the Bessel matrix defined in Proposition 2.6, and abbreviate $\boldsymbol{\beta}_{m}(1)$ as $\boldsymbol{\beta}_{m}$. Pick the matrix $\varrho_{k}$ as given in Proposition 2.7 together with the matrix $\boldsymbol{\vartheta}_{m}$ as described in Proposition 2.8. We further introduce a matrix $\boldsymbol{\Psi}_{k} \in \mathbb{Z}^{(2 k-1) \times(2 k-2)}$ with entries

$$
\left(\boldsymbol{\Psi}_{k}\right)_{a, b}= \begin{cases}\delta_{a, b}\left(1-\delta_{a, k}\right), & a \in \mathbb{Z} \cap[1, k], \\ \delta_{a, b+1}, & a \in \mathbb{Z} \cap[k+1,2 k-1] .\end{cases}
$$


The following representations of de Rham matrices hold for $k \in \mathbb{Z}_{>1}$ :

$$
\begin{aligned}
& \frac{\left|\mathcal{L}_{2 k-1}(1)\right|\left(\boldsymbol{\vartheta}_{2 k-1}^{-1}\right)^{\mathrm{T}}\left(\boldsymbol{\beta}_{2 k-1}^{-1}\right)^{\mathrm{T}} \mathbf{V}_{2 k-1}(1) \boldsymbol{\beta}_{2 k-1}^{-1} \boldsymbol{\vartheta}_{2 k-1}^{-1}}{2^{2}(2 k+1)(-1)^{k-1}}=\left(\begin{array}{ll}
\left(\frac{2}{2 k+1}\right)^{2} \mathbf{D}_{2 k-1} & \\
& \mathbf{D}_{2 k-3}
\end{array}\right), \\
& \lim _{u \rightarrow 1^{-}} \frac{\left|\mathcal{L}_{2 k}(u)\right|\left(\boldsymbol{\vartheta}_{2 k}^{-1}\right)^{\mathrm{T}} \boldsymbol{\varrho}_{k}\left(\boldsymbol{\beta}_{2 k}^{-1}\right)^{\mathrm{T}} \mathbf{V}_{2 k}(u) \boldsymbol{\beta}_{2 k}^{-1} \boldsymbol{\varrho}_{k}^{\mathrm{T}} \boldsymbol{\vartheta}_{2 k}^{-1}}{2^{3}(k+1)(-1)^{k-1}}=\left(\begin{array}{ll}
\left(\frac{1}{k+1}\right)^{2} \mathbf{D}_{2 k} & \\
& \mathbf{D}_{2 k-2}
\end{array}\right), \\
& \lim _{u \rightarrow 0^{+}} \frac{\left|\mathcal{L}_{2 k}(u)\right|\left[\boldsymbol{\beta}_{2 k}^{\mathrm{T}}(u)\right]^{-1} \mathbf{V}_{2 k}(u)\left[\boldsymbol{\beta}_{2 k}(u)\right]^{-1}}{2^{3}(-1)^{k}}=\left(\begin{array}{rr} 
& -\mathbf{D}_{2 k-1} \\
\mathbf{D}_{2 k-1} & \mathbf{D}_{2 k-1}
\end{array}\right), \\
& \lim _{u \rightarrow 0^{+}} \frac{\left|\mathcal{L}_{2 k-1}(u)\right| \boldsymbol{\Psi}_{k}^{\mathrm{T}}\left[\boldsymbol{\beta}_{2 k-1}^{\mathrm{T}}(u)\right]^{-1} \mathbf{V}_{2 k-1}(u)\left[\boldsymbol{\beta}_{2 k-1}(u)\right]^{-1} \boldsymbol{\Psi}_{k}}{2^{3}(-1)^{k}}=\left(\begin{array}{rr} 
& -\mathbf{D}_{2 k-2} \\
\mathbf{D}_{2 k-2} & \mathbf{D}_{2 k-2}
\end{array}\right),
\end{aligned}
$$

where $\mathbf{D}_{m}$ and $\left(\mathbf{B}_{m}\right)_{a, b}=2^{-3}(-1)^{\left\lfloor\frac{m}{2}\right\rfloor+1}\left(\mathscr{B}_{0}^{D}\right)_{a, b}$ [defined in (4.12)] play rôles in the following relation for $\mathbf{P}_{\mathrm{o}}$ [defined in (1.22)]:

$$
\mathbf{P}_{m} \mathbf{D}_{m} \mathbf{P}_{m}^{\mathrm{T}}=\pi \mathbf{B}_{o}+\mathbf{P}_{o} \mathbf{D}_{m} \mathbf{P}_{m}^{\mathrm{T}}-\mathbf{P}_{m} \mathbf{D}_{m} \mathbf{P}_{o}^{\mathrm{T}}
$$

Proof. According to Proposition 4.2, the left-hand of (4.76) [resp. (4.77)] is equal to

$$
\left(\begin{array}{ll}
\mathbf{P}_{m} & \\
& -\mathbf{P}_{m-2}
\end{array}\right)^{-1}\left(\begin{array}{cc}
\left(\frac{2}{m+2}\right)^{2} \mathbf{B}_{m} & \\
& \mathbf{B}_{m-2}
\end{array}\right)\left(\begin{array}{ll}
\mathbf{P}_{m}^{\mathrm{T}} & \\
& -\mathbf{P}_{m-2}^{\mathrm{T}}
\end{array}\right)^{-1}
$$

for $m=2 k-1$ (resp. $m=2 k$ ), hence expressible in terms of de Rham matrices.

In the $u \rightarrow 0^{+}$regime, we have

$$
\begin{aligned}
I_{0}(\sqrt{u} t) & =1+O\left(u t^{2}\right), \\
K_{0}(\sqrt{u} t)+\left(\gamma_{0}+\log \frac{\sqrt{u} t}{2}\right) I_{0}(\sqrt{u} t) & =O\left(u t^{2}\right), \\
\sqrt{u} t I_{1}(\sqrt{u} t) & =O\left(u t^{2}\right), \\
\sqrt{u} t\left[K_{1}(\sqrt{u} t)-\left(\gamma_{0}+\log \frac{\sqrt{u} t}{2}\right) I_{1}(\sqrt{u} t)\right] & =1+O\left(u t^{2}\right),
\end{aligned}
$$

where $\gamma_{0}$ is the Euler-Mascheroni constant.

With a reshuffling-rescaling matrix $\boldsymbol{\xi}_{2 k} \in \mathbb{Q}^{2 k \times 2 k}$ defined by (3.81), we may paraphrase [49, Proposition 4.7] into

$$
\lim _{u \rightarrow 0^{+}} \boldsymbol{\beta}_{2 k}(u) \mathbf{W}_{2 k}(u) \boldsymbol{\xi}_{2 k}^{\mathrm{T}} \boldsymbol{\gamma}_{2 k}^{\mathrm{T}}(u)=\left(\begin{array}{cc}
\sqrt{\pi} \mathbf{P}_{2 k-1}^{\mathrm{T}} & -\frac{1}{\sqrt{\pi}} \mathbf{P}_{2 k-1}^{\mathrm{T}} \\
& -\frac{1}{\sqrt{\pi}} \mathbf{P}_{2 k-1}^{\mathrm{T}}
\end{array}\right),
$$

where $\boldsymbol{\gamma}_{2 k}(u)=\left(\begin{array}{l}\mathbf{I}_{k} \\ \frac{1}{\pi}\left(\gamma_{0}+\log \frac{\sqrt{u}}{2}\right) \\ \mathbf{I}_{k} \mathbf{I}_{k}\end{array}\right)$, and $\mathbf{P}_{0} 2 k-1$ is described in 1.22). Meanwhile, by direct computation, we have

$$
\begin{aligned}
(-1)^{k} 2^{3}\left(\begin{array}{cc} 
& \mathbf{B}_{2 k-1} \\
-\mathbf{B}_{2 k-1} & \mathbf{B}_{2 k-1}
\end{array}\right) & =\boldsymbol{\xi}_{2 k} \mathbf{S}_{2 k}^{-1}(u) \boldsymbol{\xi}_{2 k}^{\mathrm{T}}=\boldsymbol{\gamma}_{2 k}(u) \boldsymbol{\xi}_{2 k} \mathbf{S}_{2 k}^{-1}(u) \boldsymbol{\xi}_{2 k}^{\mathrm{T}} \boldsymbol{\gamma}_{2 k}^{\mathrm{T}}(u) \\
& =\left|\mathcal{L}_{2 k}(u)\right| \boldsymbol{\gamma}_{2 k}(u) \boldsymbol{\xi}_{2 k} \mathbf{W}_{2 k}^{\mathrm{T}}(u) \mathbf{V}_{2 k}(u) \mathbf{W}_{2 k}(u) \boldsymbol{\xi}_{2 k}^{\mathrm{T}} \boldsymbol{\gamma}_{2 k}^{\mathrm{T}}(u) .
\end{aligned}
$$


Therefore, the limit in (4.78) exists, and is equal to

$$
\begin{aligned}
& \left(\begin{array}{cc}
\sqrt{\pi} \mathbf{P}_{2 k-1} & \\
-\frac{1}{\sqrt{\pi}} \mathbf{P}_{2 k-1} & -\frac{1}{\sqrt{\pi}} \mathbf{P}_{2 k-1}
\end{array}\right)^{-1}\left(\begin{array}{ll} 
& \mathbf{B}_{2 k-1} \\
-\mathbf{B}_{2 k-1} & \mathbf{B}_{2 k-1}
\end{array}\right)\left(\begin{array}{ll}
\sqrt{\pi} \mathbf{P}_{2 k-1}^{\mathrm{T}} & -\frac{1}{\sqrt{\pi}} \mathbf{P}_{2 k-1}^{\mathrm{T}} \\
& -\frac{1}{\sqrt{\pi}} \mathbf{P}_{2 k-1}^{\mathrm{T}}
\end{array}\right)^{-1} \\
= & \left(\begin{array}{lll}
\mathbf{D}_{2 k-1} & \mathbf{P}_{2 k-1}^{-1}\left[\pi \mathbf{B}_{2 k-1}+\mathbf{P}_{2 k-1} \mathbf{P}_{2 k-1}^{-1} \mathbf{B}_{2 k-1}-\mathbf{B}_{2 k-1}\left(\mathbf{P}_{2 k-1}^{\mathrm{T}}\right)^{-1} \mathbf{P}_{2 k-1}^{\mathrm{T}}\right] & -\mathbf{D}_{2 k-1} \\
\left(\mathbf{P}_{2 k-1}^{\mathrm{T}}\right)^{-1}
\end{array}\right) .
\end{aligned}
$$

Recalling the Broadhurst-Roberts quadratic relation $\mathbf{P}_{2 k-1} \mathbf{D}_{2 k-1} \mathbf{P}_{2 k-1}^{\mathrm{T}}=\mathbf{B}_{2 k-1}$, we may also rewrite $\mathbf{P}_{2 k-1} \mathbf{P}_{2 k-1}^{-1} \mathbf{B}_{2 k-1}-\mathbf{B}_{2 k-1}\left(\mathbf{P}_{2 k-1}^{\mathrm{T}}\right)^{-1} \mathbf{P}_{2 k-1}^{\mathrm{T}}=\mathbf{P}_{2 k-1} \mathbf{D}_{2 k-1} \mathbf{P}_{2 k-1}^{\mathrm{T}}-\mathbf{P}_{2 k-1} \mathbf{D}_{2 k-1} \mathbf{P}_{2 k-1}^{\mathrm{T}}$, thereby confirming (4.80) when $m=2 k-1$ is an odd number.

When $m=2 k$ is an even number, the ideas behind (4.80) are essentially similar, except that one needs a variation on Lemma 4 .1 to show that the $k$-th row and the $k$-th column of $\lim _{u \rightarrow 0^{+}}\left|\mathcal{L}_{2 k-1}(u)\right|\left[\boldsymbol{\beta}_{2 k-1}^{\mathrm{T}}(u)\right]^{-1}$ $\mathbf{V}_{2 k-1}(u)\left[\boldsymbol{\beta}_{2 k-1}(u)\right]^{-1}$ are filled with zeros. We leave the details to diligent readers.

Remark Equating the different representations of de Rham matrix $\mathbf{D}_{m}$, we may deduce recursive relations connecting a party of integers $D^{n} \ell_{m, j}(1), j \in \mathbb{Z} \cap[0, m]$ to another party $D^{n} \ell_{m-2, j}(1), j \in \mathbb{Z} \cap[0, m-2]$ [see (4.76) and (4.77)], as well as relations connecting both parties to $D^{n} \ell_{m-1, j}(0), j \in \mathbb{Z} \cap[0, m-1$ ] [see (4.78) and (4.79)]. These recursions on $D^{n} \ell_{m, j}$ are effectively just restatements of the following facts under suitably chosen bases: $I_{0}(t) K_{1}(t)+I_{1}(t) K_{0}(t)=\frac{1}{t}, \lim _{u \rightarrow 0^{+}} I_{0}(\sqrt{u} t)=1$ and $\lim _{u \rightarrow 0^{+}} \sqrt{u} t K_{1}(\sqrt{u} t)=1$. Direct combinatorial proofs of such recursions may appear hard, though not infeasible.

Corollary 4.7 (Anti-diagonal structure of $\mathbf{D}_{2 k-1}$ ). The de Rham matrix $\mathbf{D}_{2 k-1}$ is upper-left triangular, with its main anti-diagonal being occupied by $\left[\frac{(2 k+1) ! !}{2^{k+1}}\right]^{2}$.

Proof. After inspecting the highest order derivatives and highest order moments in $2 k$ equations generated from rows of (4.4), we immediately know that $\left[\boldsymbol{\beta}_{2 k}^{\mathrm{T}}(u)\right]^{-1}$ has the following structure:

$$
\left(\left[\boldsymbol{\beta}_{2 k}^{\mathrm{T}}(u)\right]^{-1}\right)_{a, b}= \begin{cases}0, & a=1, b \in \mathbb{Z} \cap[2,2 k], \\ (-4 u)^{1-a}, & a \in \mathbb{Z} \cap[1, k], b=2 a-1, \\ 0, & a \in \mathbb{Z} \cap[2, k], b \in \mathbb{Z} \cap[1,2 a-1), \\ \frac{1}{2 u}(-4 u)^{k+1-a}, & a \in \mathbb{Z} \cap[k+1,2 k], b=2(a-k), \\ 0, & a \in \mathbb{Z} \cap[k+1,2 k], b \in \mathbb{Z} \cap[1,2(a-k)) .\end{cases}
$$

Since $\mathbf{V}_{2 k}(u)$ is upper-left triangular, with anti-diagonal elements $\left(\mathbf{V}_{2 k}(u)\right)_{a, 2 k+1-a}=(-1)^{a-1}$, we can show that

$$
\left(\left[\boldsymbol{\beta}_{2 k}^{\mathrm{T}}(u)\right]^{-1} \mathbf{V}_{2 k}(u)\right)_{a, b}= \begin{cases}(-4 u)^{1-a}, & a \in \mathbb{Z} \cap[1, k], b=2(k-a), \\ 0, & a \in \mathbb{Z} \cap[2, k], b \in \mathbb{Z} \cap(2(k-a), 2 k], \\ -\frac{1}{2 u}(-4 u)^{k+1-a}, & a \in \mathbb{Z} \cap[k+1,2 k], b=2(2 k-a)+1, \\ 0, & a \in \mathbb{Z} \cap[k+1,2 k], b \in \mathbb{Z} \cap(2(2 k-a)+1,2 k] .\end{cases}
$$

This further leads us to a block partition $\left[\boldsymbol{\beta}_{2 k}^{\mathrm{T}}(u)\right]^{-1} \mathbf{V}_{2 k}(u)\left[\boldsymbol{\beta}_{2 k}(u)\right]^{-1}=\left(\begin{array}{c}* \boldsymbol{U} \\ { }_{-\boldsymbol{U}^{\mathrm{T}}} *\end{array}\right)$ where $\boldsymbol{U} \in \mathbb{Q}(u)^{k \times k}$ is an upper-left triangular matrix whose main anti-diagonal is occupied by $\frac{(-1)^{k-1}}{2^{2 k-1} u^{k}}$. (Here, an asterisk denotes a block with irrelevant details.) Referring back to (4.78), we know that $\mathbf{D}_{2 k-1}$ is an upper-left triangular matrix whose main anti-diagonal is filled with

$$
\lim _{u \rightarrow 0^{+}} \frac{(-1)^{k-1}}{2^{2 k-1} u^{k}} \frac{\left|\mathcal{L}_{2 k}(u)\right|}{2^{3}(-1)^{k-1}}=\left[\frac{(2 k+1) ! !}{2^{k+1}}\right]^{2},
$$

as claimed. 
Remark In [25, Theorem 1.4], Fresán, Sabbah and Yu have characterized (effectively) the inverse de Rham matrices $\mathbf{D}_{2 k-1}^{-1}, k \in \mathbb{Z}_{>0}$ and $\mathbf{D}_{2 k}^{-1}, k \in 2 \mathbb{Z}_{>0}$, in the form of lower-right triangular matrices, with explicit formulae for elements on the main anti-diagonal. Paraphrasing their result with matrix inversions and suitable normalizations, we can also confirm the anti-diagonal behavior of $\mathbf{D}_{2 k-1}$.

Extending the proof the proposition above, one can show that the de Rham matrix $\mathbf{D}_{2 k}$ is always upper-left triangular. When $k$ is odd, the center of the main anti-diagonal in the skew-symmetric matrix $\mathbf{D}_{2 k}=\mathbf{P}_{2 k}^{-1} \mathbf{B}_{2 k}\left(\mathbf{P}_{2 k}^{\mathrm{T}}\right)^{-1}$ must be zero, hence $\operatorname{det} \mathbf{D}_{2 k}=0$. (Alternatively, one may deduce this fact from $\operatorname{det} \mathbf{B}_{2 k}=0$, which has been proved in Corollary 4.3 ,)

To avoid inverting a singular de Rham matrix, Fresán-Sabbah-Yu [25, §3.b] constructed a quadratic relation that was different from $\mathbf{P}_{2 k} \mathbf{D}_{2 k} \mathbf{P}_{2 k}^{\mathrm{T}}=\mathbf{B}_{2 k}$ for odd $k$, without involving the last row in $\mathbf{P}_{2 k}$. Accordingly, the Gröbner basis generating their quadratic relation does not contain linear sum rules derivable from generalized Crandall numbers (see (2.47), which recapitulates [53, (65)]).

The uniqueness of de Rham matrices gives an indirect proof that our constructions of $\mathbf{D}_{2 k-1}, k \in \mathbb{Z}_{>0}$ and $\mathbf{D}_{2 k}, k \in 2 \mathbb{Z}_{>0}$ are equivalent to Fresán-Sabbah-Yu [25, §3.b]. Without resorting to a uniqueness argument, we may interpret such equivalence directly through the following identities (cf. [49, (4.22)] or [54, (2.10]):

$$
\left\{\begin{array}{c}
\widetilde{L}_{m}\left(u D^{2}+D^{1}\right) \frac{I_{0}(\sqrt{u} t)}{t}=\frac{(-1)^{m}}{2^{m+2}} L_{m+2}^{*} \frac{I_{0}(\sqrt{u} t)}{t}, \\
\widetilde{L}_{m}\left(u D^{2}+D^{1}\right) \frac{K_{0}(\sqrt{u} t)}{t}=\frac{(-1)^{m}}{2^{m+2}} L_{m+2}^{*} \frac{K_{0}(\sqrt{u} t)}{t} .
\end{array}\right.
$$

Here, the operator $L_{m+2}^{*}$ is formal adjoint to the Borwein-Salvy operator $L_{m+2}$ [6], Lemma 3.3], which is the $(m+1)$-st symmetric power of the Bessel differential operator $\left(t \frac{\mathrm{d}}{\mathrm{d} t}\right)^{2}-t^{2}\left(t \frac{\mathrm{d}}{\mathrm{d} t}\right)^{0}$, annihilating each member in the set $\left\{\left[I_{0}(t)\right]^{j}\left[K_{0}(t)\right]^{m+1-j} \mid j \in \mathbb{Z} \cap[0, m+1]\right\}$. The Borwein-Salvy operator $L_{m+2}=\mathscr{L}_{m+2, m+2}$ is constructed recursively by the Bronstein-Mulders-Weil algorithm [15, Theorem 1]:

$$
\left\{\begin{array}{l}
\mathscr{L}_{m+2,0}=\left(t \frac{\mathrm{d}}{\mathrm{d} t}\right)^{0}, \mathscr{L}_{m+2,1}=t \frac{\mathrm{d}}{\mathrm{d} t}, \\
\mathscr{L}_{m+2, k+1}=t \frac{\mathrm{d}}{\mathrm{d} t} \mathscr{L}_{m+2, k}-k(m+2-k) t^{2} \mathscr{L}_{m+2, k-1}, \quad \forall k \in \mathbb{Z} \cap[1, m+1] .
\end{array}\right.
$$

To find out the de Rham matrix that fits into the relation $\mathbf{P}_{m} \mathbf{D}_{m}=\mathbf{B}_{m} \frac{\operatorname{cof} \mathbf{P}_{m}}{\operatorname{det} \mathbf{P}_{m}}$, we have differentiated offshell Bessel moments with respect to $u$, and have evaluated these derivatives in the $u \rightarrow 1^{-}$limit. To attain the same goal, Fresán, Sabbah and Yu [25, Proposition 3.4] have integrated by parts with respect to $t$, relating Bessel moments $\operatorname{IKM}(a, b ; n)$ of different orders $n$ by a recursive algorithm [25, (3.9)] that emulates Bronstein-Mulders-Weil [15, Theorem 1] and Borwein-Salvy [6, Lemma 3.3]. In other words, the equivalence between our Wrońskian approach to de Rham matrices and the non-Wrońskian approach of Fresán-Sabbah-Yu [25, §3.b] ultimately roots from the duality relation (4.92) between Vanhove and Borwein-Salvy operators, at $u=1$. We will not further elaborate on such a duality, as it is not essential to the theme of the current work.

Broadhurst and Roberts have proposed a set of combinatorial formulae [13, (5.7)-(5.12)] that conjecturally generate the de Rham matrices $\mathbf{D}_{m}, m \in \mathbb{Z}_{>0}$ through a recursion on $m$. The uniqueness argument alone does not allow us to settle their conjecture. What we currently know is that various representations of $\mathbf{D}_{m}$ in Proposition 4.6 do provide us with recursive relations concerning special values of the polynomials $D^{n} \ell_{m, j}$ with three consecutive values of $m$, which are reminiscent of the three-term recursion for the Broadhurst-Roberts bivariate polynomials [13, (5.10)]. Perhaps the former three-term relations can be translated into the Broadhurst-Roberts recursions for $\mathbf{D}_{m}$, through some combinatorial efforts.

It is our hope that the recursive structures of $\mathbf{D}_{m}$, once completely understood, will shed new light on the algebraic and arithmetic nature of the Broadhurst-Roberts ideal $I_{m+2}^{\mathrm{BR}}=\left\langle\mathbf{X}_{\lfloor(m+1) / 2\rfloor} \mathbf{D}_{m} \mathbf{X}_{\lfloor(m+1) / 2\rfloor}^{\mathrm{T}}-\right.$ $\left.\mathbf{B}_{m}\right\rangle$ and the corresponding Broadhurst-Roberts variety $V_{m+2}^{\mathrm{BR}}:=\left\{\mathbf{X}_{\lfloor(m+1) / 2\rfloor} \in \mathbb{C}^{\left\lfloor\frac{m+1}{2}\right\rfloor \times\left\lfloor\frac{m+1}{2}\right\rfloor} \mid \mathbf{X}_{\lfloor(m+1) / 2\rfloor} \mathbf{D}_{m}\right.$ $\left.\mathbf{X}_{\lfloor(m+1) / 2\rfloor}^{\mathrm{T}}-\mathbf{B}_{m}=0\right\}$. 


\section{REFERENCES}

[1] Kevin Acres and David Broadhurst. Empirical determinations of Feynman integrals using integer relation algorithms. arXiv:2103.06345v1 [hep-ph], 2021.

[2] Takashi Agoh and Karl Dilcher. Reciprocity relations for Bernoulli numbers. Amer. Math. Monthly, 115(3):237-244, 2008.

[3] David H. Bailey, Jonathan M. Borwein, David Broadhurst, and M. L. Glasser. Elliptic integral evaluations of Bessel moments and applications. J. Phys. A, 41(20):205203 (46pp), 2008. arXiv:0801.0891v2 [hep-th].

[4] Tadeusz Banachiewicz. Zur Berechnung der Determinanten, wie auch der Inversen, und zur darauf basierten Auflösung der Systeme linearer Gleichungen. Acta Astronomica, Series C, 3:41-67, 1937.

[5] Spencer Bloch, Matt Kerr, and Pierre Vanhove. A Feynman integral via higher normal functions. Compos. Math., 151(12):2329-2375, 2015. arXiv:1406.2664v3 [hep-th].

[6] Jonathan M. Borwein and Bruno Salvy. A proof of a recurrence for Bessel moments. Experiment. Math., 17(2):223-230, 2008. arXiv:0706.1409v2 [cs.SC].

[7] Jonathan M. Borwein, Armin Straub, James Wan, and Wadim Zudilin. Densities of short uniform random walks. Canad. J. Math., 64(5):961-990, 2012. (With an appendix by Don Zagier) arXiv:1103.2995 [math.CA].

[8] David Broadhurst. Reciprocal PSLQ and the tiny nome of Bologna. In International Workshop "Frontiers in Perturbative Quantum Field Theory", Bielefeld, Germany, June 14, 2007. Zentrum für interdisziplinäre Forschung in Bielefeld. https://www.researchgate.net/publication/266404058_Reciprocal_PSLQ_and_the_tiny_Nome_of_

[9] David Broadhurst. Feynman integrals, L-series and Kloosterman moments. Commun. Number Theory Phys., 10(3):527-569, 2016. arXiv:1604.03057v1 [physics.gen-ph].

[10] David Broadhurst. L-series from Feynman diagrams with up to 22 loops. In Workshop on Multi-loop Calculations: Methods and Applications, Paris, France, June 7, 2017. Séminaires Internationaux de Recherche de Sorbonne Universités. https://multi-loop-2017.sciencesconf .org/data/program/Broadhurst.pdf.

[11] David Broadhurst. Feynman integrals, quasi-periods and black holes. In Elliptics and Beyond (MITP Virtual Workshop), Mainz, Germany, September 10, 2020. Mainz Institute for Theoretical Physics.

[12] David Broadhurst and Anton Mellit. Perturbative quantum field theory informs algebraic geometry. In Loops and Legs in Quantum Field Theory. PoS (LL2016) 079, 2016. https://pos.sissa.it/archive/conferences/260/079/LL2016_079.pdf.

[13] David Broadhurst and David Roberts. Quadratic relations between Feynman integrals. In Loops and Legs in Quantum Field Theory. PoS (LL2018) 053, 2018. https://pos.sissa.it/303/053/pdf.

[14] David Broadhurst and David P. Roberts. L-series and Feynman integrals. In David R. Wood, Jan de Gier, Cheryl E. Praeger, and Terence Tao, editors, 2017 MATRIX Annals, volume 2 of MATRIX Book Series, pages 401-403. Springer, Cham, Switzerland, 2019.

[15] Manuel Bronstein, Thom Mulders, and Jacques-Arthur Weil. On symmetric powers of differential operators. In Proceedings of the 1997 International Symposium on Symbolic and Algebraic Computation (Kihei, HI), pages 156-163. ACM, New York, 1997.

[16] Bruno Buchberger. An algorithm for finding the basis elements of the residue class ring of a zero dimensional polynomial ideal. J. Symbolic Comput., 41(3-4):475-511, 2006. (Translated from the 1965 German original by Michael P. Abramson).

[17] Kilian Bönisch, Fabian Fischbach, Albrecht Klemm, Christoph Nega, and Reza Safari. Analytic structure of all loop banana amplitudes. arXiv:2008.10574 [hep-th], 2020.

[18] Philip Candelas, Xenia de la Ossa, Mohamed Elmi, and Duco van Straten. A one parameter family of Calabi-Yau manifolds with attractor points of rank two. J. High Energy Phys., 2020(10), 2020. 
arXiv:1912.06146v1 [math.NT].

[19] P. Deligne. Valeurs de fonctions $L$ et périodes d'intégrales. In Automorphic forms, representations and L-functions (Proc. Sympos. Pure Math., Oregon State Univ., Corvallis, Ore., 1977), Part 2, Proc. Sympos. Pure Math., XXXIII, pages 313-346. Amer. Math. Soc., Providence, R.I., 1979. (With an appendix by N. Koblitz and A. Ogus).

[20] Plamen Djakov and Boris Mityagin. Asymptotics of instability zones of Hill operators with a two term potential. C. R. Math. Acad. Sci. Paris, 339(5):351-354, 2004.

[21] Plamen Djakov and Boris Mityagin. Asymptotics of instability zones of the Hill operator with a two term potential. J. Funct. Anal., 242(1):157-194, 2007. arXiv:math-ph/0509034v1.

[22] Javier Fresán and Peter Jossen. Exponential motives. javier.fresan.perso.math.cnrs.fr/expmot.pdf, 2020.

[23] Javier Fresán, Claude Sabbah, and Jeng-Daw Yu. Hodge theory of Kloosterman connections. arXiv:1810.0654v3 [math.AG], 2020.

[24] Javier Fresán, Claude Sabbah, and Jeng-Daw Yu. Quadratic relations between periods of connections. arXiv:2005.11525v2 [math.AG], 2020.

[25] Javier Fresán, Claude Sabbah, and Jeng-Daw Yu. Quadratic relations between Bessel moments. arXiv:2006.02702v1][math.AG], 2020.

[26] Lei Fu and Daqing Wan. L-functions of symmetric products of the Kloosterman sheaf over $\mathbf{Z}$. Math. Ann., 342(2):387-404, 2008.

[27] M. B. Gel'fand. A note on a certain relation among Bernoulli numbers. Baškir. Gos. Univ. Učen. Zap. Vyp., 31(Ser. Mat. 3):215-216, 1968.

[28] M. L. Glasser and E. Montaldi. Staircase polygons and recurrent lattice walks. Phys. Rev. E, 48:R2339-R2342, 1993.

[29] S. Groote, J. G. Körner, and A. A. Privovarov. On the evaluation of a certain class of Feynman diagrams in $x$-space: Sunrise-type topologies at any loop order. Ann. Phys., 322:2374-2445, 2007. arXiv: hep-ph/0506286v1.

[30] K. Hulek, J. Spandaw, B. van Geemen, and D. van Straten. The modularity of the Barth-Nieto quintic and its relatives. Adv. Geom., 1(3):263-289, 2001. arXiv:math/0010049v1][math.AG].

[31] E. L. Ince. Ordinary differential equations. Dover Publications, New York, NY, 1956.

[32] S. Laporta. Analytical expressions of three- and four-loop sunrise Feynman integrals and four-dimensional lattice integrals. Internat. J. Modern Phys. A, 23(31):5007-5020, 2008. arXiv:0803.1007v4 [hep-ph].

[33] S. Laporta and E. Remiddi. The analytical value of the electron $(g-2)$ at order $\alpha^{3}$ in QED. Physics Letters B, 379(1):283-291, 1996. arXiv: hep-ph/9602417.

[34] Stefano Laporta. High-precision calculation of the 4-loop contribution to the electron $g-2$ in QED. Physics Letters B, 772(Supplement C):232-238, 2017. arXiv:1704.06996 [hep-th].

[35] Roman N. Lee and Andrei A. Pomeransky. Differential equations, recurrence relations, and quadratic constraints for L-loop two-point massive tadpoles and propagators. J. High Energy Phys., (8):027, 26, 2019. arXiv:1904.12496v1 [hep-ph].

[36] Niels Nielsen. Traité élémentaire des nombres de Bernoulli. Gauthier-Villars, Paris, France, 1923.

[37] C. Peters, J. Top, and M. van der Vlugt. The Hasse zeta function of a $K 3$ surface related to the number of words of weight 5 in the Melas codes. J. Reine Angew. Math., 432:151-176, 1992.

[38] M. Rogers, J. G. Wan, and I. J. Zucker. Moments of elliptic integrals and critical $L$-values. $R a$ manujan J., 37(1):113-130, 2015.

[39] Louis Saalschütz. Verkürzte Recursionsformeln für die Bernoullischen Zahlen. Zeit. für Math. und Phys., 37:374-378, 1892.

[40] Louis Saalschütz. Vorlesungen über die Bernoullischen Zahlen, ihren Zusammenhang mit den Secanten-Coefficienten und ihre wichtigeren Anwendungen. Springer, Berlin, Germany, 1893.

[41] Detchat Samart. Feynman integrals and critical modular L-values. Commun. Number Theory Phys., 10(1):133-156, 2016. arXiv:1511.07947v2] [math.NT]. 
[42] Pierre Vanhove. The physics and the mixed Hodge structure of Feynman integrals. In String-Math 2013, volume 88 of Proc. Sympos. Pure Math., pages 161-194. Amer. Math. Soc., Providence, RI, 2014. arXiv:1401.6438 [hep-th].

[43] Pierre Vanhove. Feynman integrals, toric geometry and mirror symmetry. In Johannes Blümlein, Carsten Schneider, and Peter Paule, editors, Elliptic integrals, elliptic functions and modular forms in quantum field theory, Texts \& Monographs in Symbolic Computation, chapter 17, pages 415458. Springer, Cham, Switzerland, 2019. arXiv:1807.11466v2 [hep-th].

[44] Pierre Vanhove. Feynman integrals and mirror symmetry. In Valery A. Gritsenko and Vyacheslav P. Spiridonov, editors, Partition Functions and Automorphic Forms, Moscow Lectures 5, pages 319367. Springer, Cham, Switzerland, 2020.

[45] H. A. Verrill. Sums of squares of binomial coefficients, with applications to Picard-Fuchs equations. arXiv: math/0407327v1 [math.CO], 2004.

[46] G. N. Watson. A Treatise on the Theory of Bessel Functions. Cambridge University Press, Cambridge, UK, 2nd edition, 1944.

[47] Zhiwei Yun. Galois representations attached to moments of Kloosterman sums and conjectures of Evans. Compos. Math., 151(1):68-120, 2015. (Appendix B by Christelle Vincent) arXiv: 1308.3920v2 [math.NT].

[48] Yajun Zhou. Wick rotations, Eichler integrals, and multi-loop Feynman diagrams. Commun. Number Theory Phys., 12(1):127-192, 2018. arXiv:1706.08308v4 [math.NT].

[49] Yajun Zhou. Wrońskian factorizations and Broadhurst-Mellit determinant formulae. Commun. Number Theory Phys., 12(2):355-407, 2018. arXiv:1711.01829v2 [math.CA].

[50] Yajun Zhou. Hilbert transforms and sum rules of Bessel moments. Ramanujan J., 48(1):159-172, 2019. arXiv:1706.01068v2 [math.CA].

[51] Yajun Zhou. On Borwein's conjectures for planar uniform random walks. J. Aust. Math. Soc., 107(3):392-411, 2019. arXiv:1708.02857v2 [math.CA].

[52] Yajun Zhou. On Laporta's 4-loop sunrise formulae. Ramanujan J., 50(3):465-503, 2019. arXiv:1801.02182v1 [math.CA].

[53] Yajun Zhou. Some algebraic and arithmetic properties of Feynman diagrams. In Johannes Blümlein, Carsten Schneider, and Peter Paule, editors, Elliptic Integrals, Elliptic Functions and Modular Forms in Quantum Field Theory, Texts \& Monographs in Symbolic Computation, chapter 19, pages 485-509. Springer, Cham, Switzerland, 2019. arXiv:1801.05555v2 [math.NT].

[54] Yajun Zhou. Q-linear dependence of certain Bessel moments. Ramanujan J., 2021. doi:10.1007/s11139-021-00416-9 (to appear) arXiv:1911.04141v3 [math.NT].

Program in Applied and Computational Mathematics (PACM), Princeton University, Princeton, nj 08544

Email address: yajunz@math.princeton.edu

Current address: ACADEMy of Advanced IntERdisciplinary Studies (AAIS), PEKING UNIVERsity, BEIJING 100871, P. R. CHINA

Email address: yajun.zhou.1982@pku.edu.cn 